Groups Geom. Dyn. 9 (2015), 369-434

DOI $10.4171 /$ GGD/317
Groups, Geometry, and Dynamics

(C) European Mathematical Society

\title{
Non-finitely generated relatively hyperbolic groups and Floyd quasiconvexity
}

\author{
Victor Gerasimov and Leonid Potyagailo
}

\begin{abstract}
We regard a relatively hyperbolic group as a group acting non-trivially by homeomorphisms on a compactum $T$ discontinuously on the set of distinct triples and cocompactly on the set of distinct pairs of points of $T$.

In the first part of the paper we prove that such a group $G$ admits a graph of groups decomposition given by a star graph whose central vertex group is finitely generated relatively hyperbolic with respect to the edge groups, and the other vertex groups are stabilizers of non-equivalent parabolic points. It follows from this result that every relatively hyperbolic group is relatively finitely generated with respect to the parabolic subgroups. Another corollary is that the definition of the relative hyperbolicity which we are using is equivalent to those of Bowditch and Osin (taken with respect to finitely many peripheral subgroups) and they are all equivalent to the existence of the above star graph of groups decomposition.

The second part of the paper uses the method of the first part. Considering the induced action of $G$ on the space of distinct pairs of $T$ we construct a connected graph on which $G$ acts properly and cofinitely on edges. Equipping the graph with Floyd metrics we prove that the quasigeodesics in this metric are close somewhere to the geodesics in the word metric. This allows us to prove that the parabolic subgroups of $G$ are quasiconvex with respect to the Floyd metrics. As a corollary we prove that the preimage of a parabolic point by the Floyd map is the Floyd boundary of its stabilizer.
\end{abstract}

Mathematics Subject Classification (2010). Primary 20F65, 20F67; Secondary 57M07, $22 \mathrm{D} 05$.

Keywords. Relatively hyperbolic group, relatively finitely generated group, Floyd quasiconvexity. 


\section{Contents}

1 Introduction . . . . . . . . . . . . . . . . . . . . . 370

2 Convergence groups . . . . . . . . . . . . . . 378

3 Exhaustion of non-finitely generated relatively hyperbolic groups by finitely generated ones . . . . . . . . . . . . . . . . . 379

4 Floyd metrics and shortcut metrics . . . . . . . . . . . . . . . . 410

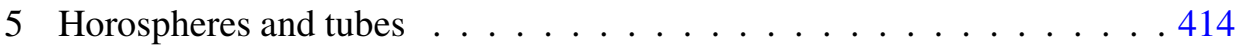

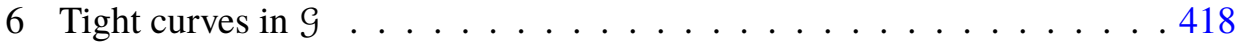

7 Floyd quasiconvexity of parabolic subgroups . . . . . . . . . . . 425

References . . . . . . . . . . . . . . . . . . . 432

\section{Introduction}

First part of the paper. Let $T$ be a compact Hausdorff space (compactum) containing at least 3 points. The action of a discrete group $G$ by homeomorphisms of $T$ is called convergence action if the induced action on the space $\Theta^{3} T$ of subsets of cardinality 3 is discontinuous. We say in this case that the action is 3-discontinuous.

The action of $G$ on $T$ is called 2-cocompact if the action on $\Theta^{2} T$ is cocompact. An action is called parabolic if $G$ is infinite there is a unique fixed point.

If $G$ admits a non-parabolic action on $T$ which is 3-discontinuous and 2-cocompact then the action is geometrically finite, i.e. every point of $T$ is either conical or bounded parabolic or isolated [15]. Conversely if a group $G$ admits a minimal geometrically finite action on a metrisable space $T$ without isolated points then the action is 2-cocompact [29]. If $G$ is finitely generated then the existence of a geometrically finite action of $G$ is equivalent (see [2] and [31]) to the "classical" relative hyperbolicity in the sense of Farb [11] and Gromov $[20,8.6]$.

These facts justify the following "dynamical" definition.

Definition 1.1 ([15]). A group $G$ is called relatively hyperbolic if it admits a non-parabolic 3-discontinuous and 2-cocompact action $\left(\mathrm{RH}_{32}\right.$-action) on a compactum $T$.

We point out that we do not impose any restriction on the cardinality of $G$. We also do not require the metrisability of $T$.

Our first result shows that any relatively hyperbolic group can be "nicely" approximated by finitely generated relatively hyperbolic group. 
Theorem A. Let $G$ be a relatively hyperbolic group with respect to a collection of parabolic subgroups $\left\{P_{1}, \ldots, P_{n}\right\}$. Then $G$ is the fundamental group of the following finite "star graph"

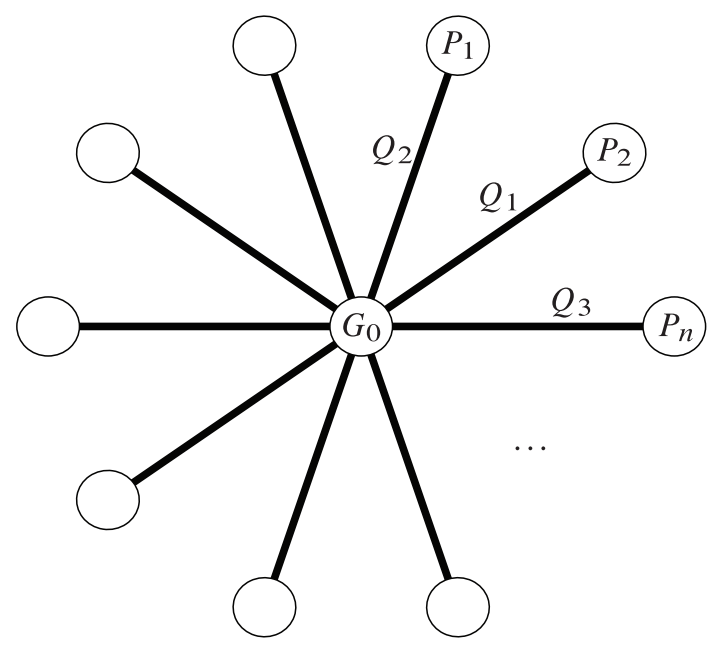

whose central vertex group $G_{0}$ is finitely generated relatively hyperbolic with respect to those edge groups $Q_{i}=P_{i} \cap G_{0}$ which are infinite, all other vertex groups of the graph are $P_{i}(i=1, \ldots, n)$.

Moreover for every finite set $K \subset G$ the subgroup $G_{0}$ can be chosen to contain $K$.

Theorem A yields generalization of several known results omitting the assumption of finite generatedness.

A group $G$ is said to be finitely generated with respect to a collection $\mathcal{H}$ of subgroups if there exists a finite set $S \subset G$ such that $S \cup(\cup \mathcal{H})$ is a generating set for $G$.

Corallary (Corollaries 3.39, 3.40). Let a group $G$ admit a 3-discontinuous 2 -cocompact non-parabolic action on a compactum $T$. Then $G$ is finitely generated with respect to a finite collection of the stabilizers of parabolic points. In particular, if $G$ acts without parabolics then $G$ is finitely generated.

In [17, Appendix] we gave a short proof of Bowditch's theorem that the existence of a 3-discontinuous and 3-cocompact action of a finitely generated group implies that the group is hyperbolic. The above Corollary omits the assumption of finite generatedness. 
In most papers about relatively hyperbolic groups the authors assume that the group is finitely generated. Besides Definition 1.1 there are two more definitions which do not require the finite generatedness. The first is due to B. Bowditch [2] and the second is due to D. Osin [26]. We recall them now.

A graph $\Gamma$ is called fine if for any two vertices the set of arcs of fixed length joining them is finite. Bowditch calls a group $G$ relatively hyperbolic if there is an action of $G$ on a fine hyperbolic graph $\Gamma$ such that the action on edges is proper (i.e. the edge stabilizers are finite), cofinite (the set of edge orbits is finite i.e. $\left|\Gamma^{1} / G\right|<\infty$ ) and non-parabolic (there is no vertex fixed by $G$ ).

We use a "finite" version of Osin's definition of relative hyperbolicity according to which a group $G$ is relatively hyperbolic with respect to a finite collection $\mathfrak{P}$ of subgroups of infinite index, if it is relatively finitely presented with respect to $\mathfrak{P}$ and satisfies linear isoperimetric inequality relative to this system (see Definition 3.42 in Section 3.5). We note that the original Osin's definition does not require the finiteness of the peripheral system $\mathfrak{P}$ whereas all other definitions imply this property. So to relate Osin's definition with all other definitions we will always assume that the system $\mathfrak{P}$ is finite. The assumption that every element of $\mathfrak{P}$ is a subgroup of infinite index is needed to exclude the trivial case of the relative hyperbolicity with respect to a subgroup of finite index. The existence of the star graph decomposition (1) directly follows from Osin's definition (see [26, Theorem 2.44]). Our Theorem A is a different result as it uses another definition of the relative hyperbolicity. On its turn since the existence of such a graph of groups decomposition is a common point for both these approaches it gives rise to the following equivalence of all known definitions of the relative hyperbolicity valid for a group without any restriction on its cardinality.

Corollary (Theorem 3.1). The following conditions of the relative hyperbolicity for a group $G$ are equivalent.

1) (The above Definition 1.1) The group $G$ admits a $\mathrm{RH}_{32}$-action on a compactum $T$ containing at least 3 points.

2) (Bowditch's definition) The group $G$ acts non-parabolically on a connected fine hyperbolic graph $\Gamma$ properly and cofinitely on edges.

3) (Osin's definition) The group $G$ is relatively finitely presented and admits a relative linear isoperimetric inequality relatively to a finite system of subgroups of infinite index.

4) $G$ admits the star graph decomposition (1) where the central vertex group $G_{0}$ is a finitely generated relatively hyperbolic group with respect to those edge groups $Q_{i}$ which are infinite. 
The implication 1) $\Longrightarrow$ 4) follows from Theorem A and 3) $\Longrightarrow$ 4) from [26, Theorem 2.44]. The implication 2) $\Longrightarrow 1$ ) is proved in [16]. The following proposition yields the implication 4) $\Longrightarrow 2$ ).

Proposition 3.43. Suppose that a group $G$ admits a graph of groups decomposition (1) where the group $G_{0}$ is finitely generated and relatively hyperbolic with respect to the subgroups $Q_{i}(i=1,,, n)$. Then $G$ satisfies Bowditch's definition.

The proof of the implication 2) $\Longrightarrow 3$ ) is an easy use of the common methods for hyperbolic metric spaces [20]. We include it for the completeness avoiding the references to the sources in which it is not clear that the assumption of finite generatedness is inessential.

We resume all this discussion in the following diagram:

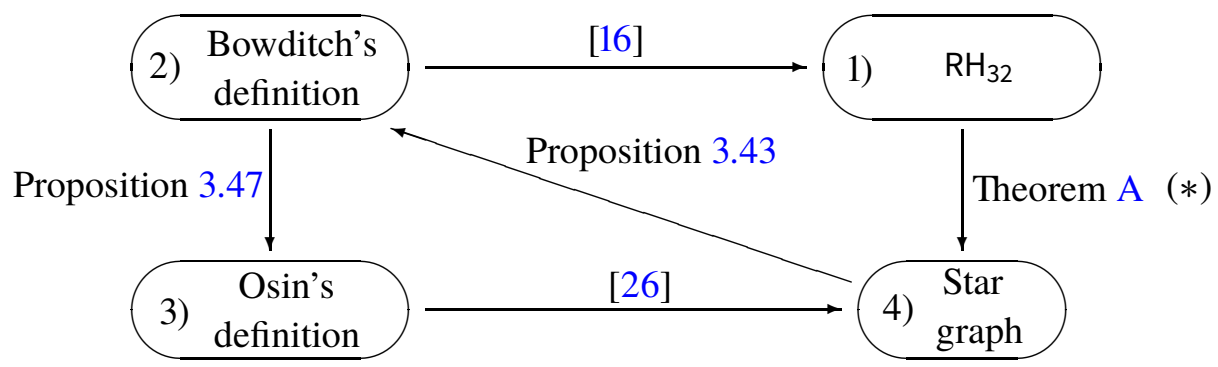

We note that the vertex groups corresponding to the non-central vertices of the star graph in the above Proposition 3.43 can be uncountable. This provides a construction of an uncountable relatively hyperbolic group too. The proof of Theorem A presented below does not depend on the cardinality of $G$ nor on the metrisability of the space on which it acts, and is self-contained. It is based on the theory of entourages of a compactum $T$ which are the neighborhoods of the diagonal of $T^{2}$. In Section 3 using a $G$-orbit $A$ of entourages on $T$ we construct a graph $\mathcal{G}$ on which $G$ acts and whose set of vertices is $A$. The subgroup $G_{0}$ will be chosen as the stabilizer of a connected component of a refined graph $\widetilde{\mathcal{G}}$ having the same set of vertices: $\widetilde{\mathcal{G}}^{0}=\mathcal{G}^{0}=A$. We will use a system of tubes and horospheres on $\mathcal{G}$ to establish the existence of the requested splitting of $G$ as a star-graph of groups. 
Historical remarks and comments. For the completeness of the exposition we provide a short survey of known results related to Theorem 3.1. We start with a less general (but more standard) case of a finitely generated group and then describe briefly what is known when $G$ is a non-finitely generated group.

Case 1. G is Finitely generated. The equivalence of conditions 2) and 3) was proved by F. Dahmani [9] and D. Osin [26].

B. Bowditch proved that condition 2) implies that $G$ acts properly discontinuously by isometries on a proper hyperbolic metric space $X$, and the action on the boundary $\partial X$ is geometrically finite meaning that every point of $\partial X$ is either conical or bounded parabolic. A strengthened converse statement was proved by A. Yaman [31]. She showed that a group that possesses a geometrically finite convergence action on a non-empty metrisable perfect compactum $T$ such that the stabilizers of parabolic points are all finitely generated satisfies condition 2). Note that the finite generatedness of the maximal parabolic subgroups implies by Corollary 3.39 that the whole group $G$ is finitely generated.

From the other hand a minimal action on a metrisable compactum is an $\mathrm{RH}_{32}$-action if and only if it is geometrically finite. Indeed the sufficiency follows from P. Tukia's result [29, Theorem 1.C]. The converse statement is a partial case of [15, Main Theorem, b].

So conditions 1), 2) and 3) are equivalent if $G$ is finitely generated. By [26, Lemma 2.46] the implication 4) $\Longrightarrow 3$ ) is true for any $G$ (we thank the referee for this reference). On its turn 4) trivially holds for every finitely generated relatively hyperbolic group.

Note that an alternative proof of Yaman's theorem in the finitely generated case is given in [18, Corollary of 7.1.1].

Case 2. G is countable. In [23] C. Hruska pointed out that the proofs of the equivalence between conditions 2) and 3) given in [9] and [26] remain true for countable groups. However their relation with the geometrical finitenness is more delicate already in this case. C. Hruska noticed that the proof of the above theorem of Bowditch does not work if the parabolic subgroups are not finitely generated [23, Remark after 5.6]. He indicated how to generalize the methods of the paper of D. Groves and J.F. Manning [22] and to prove the implication 3) $\Longrightarrow 2$ ) (in fact the argument gives a stronger statement that 3) implies Gromov's definition of the relative hyperbolicity denoted by (RH-3) in [23]).

It is claimed without proof in [23] that Yaman's theorem remains valid in the countable case (note that in [23] this statement was misleadingly denoted $(\mathrm{RH}-1) \Longrightarrow(\mathrm{RH}-2)$ but it should be $(\mathrm{RH}-1) \Longrightarrow(\mathrm{RH}-4))$. The main part of 
Yaman's proof consists in generalizing the statements of the paper [4] about hyperbolic groups to the case of relatively hyperbolic groups. In particular Yaman uses some lemmas of [4] when the group is a posteriori finitely generated. So it seems to us that these arguments still require further explanations in the case when the group is not finitely generated.

Note that if one admits that the proof of Yaman works in the countable case then it would give a proof of our Theorem A in this case. Indeed 1) yields that the action is geometrically finite by [15]. Then Yaman's theorem would imply 2). Finally we obtain 2) $\Longrightarrow 3$ ) as it is described in [23] and 3) $\Longrightarrow$ 4) by [26, Theorem 2.44].

The argument of Hruska generalizing the theorem of [22] requires the metrisability of the compactum $T$ which is homeomorphic to the boundary of a hyperbolic space (given by Gromov's definition (RH3)). Any group $G$ admitting a 3-discontinuous action on the metrisable compactum $T$ is countable [15, Corollary 2, Section 5.3] (note that the converse statement is true if one supposes that the action of $G \curvearrowright T$ is $\mathrm{RH}_{32}$ then the countability of $G$ implies the metrisability of $T$ [15, Main Theorem, c]). So the condition to be countable for a relatively hyperbolic group seems to be unavoidable in this approach.

Case 3. $G$ is an arbitrary group. As we have mentioned the equivalence 3) $\Longleftrightarrow 4$ ) is true for any group [26, 2.44 and 2.46]. The proof of the equivalence of conditions 3 ) and 4) to condition 2) is not so difficult. Since the arguments are spread in different papers and sometimes require modifications, we included them in Propositions 3.43 and 3.47.

The relation with the dynamical condition $\mathrm{RH}_{32}$ (or with the geometrical finitenness) was not known before. Thus the main result of the section is Theorem A which establishes (with the statement 2) $\Longrightarrow 1$ ) from [16]) the equivalence of condition 1) to all other conditions.

One of the difficulties of the situation is that the condition $\mathrm{RH}_{32}$ still implies the geometrically finiteness by [15] but the converse statement is not known in this case (the argument of Tukia certainly needs the metrisability of the compactum on which the group acts).

The implication 1) $\Longrightarrow 3$ ) follows from the above Corollary. It generalizes Yaman's theorem to the case of an arbitrary group admitting an $\mathrm{RH}_{32}$-action (see also [18, Proposition 7.1.2]). In particular if $G$ is countable together with Tukia's theorem it yields a proof of Yaman's theorem in this case. 
We note that despite that Osin's theorem and Theorem A have the same conclusion (condition 4)) and their assumptions (conditions 3) and 1) respectively) are equivalent, none of them is a corollary of the other one as the proof of this equivalence uses both statements.

Notice also that the star-graph decompositions of relatively hyperbolic groups have been used in [26] to reduce the case of a non-finitely generated relatively finitely presented group to the case of a finitely generated one.

We finish this discussion by the following question asking whether Tukia's theorem remains valid without assuming the metrisability of the space.

Question. Is it true that a geometrically finite non-elementary minimal action on a compactum is an $\mathrm{RH}_{32}$-action?

A positive answer to this question would imply in particular that Yaman's theorem is true in the non-metrisable case too.

Second part of the paper. It deals with finitely generated relatively hyperbolic groups. It is based on the methods developed in the first part. Starting with Section 4 we use the Floyd completion of locally finite graphs. Let $\Gamma$ be a locally finite, connected graph admitting a cocompact and discontinuous action of a finitely generated group $G$ (e.g. a Cayley graph of $G$ or the graph of entourages $\mathcal{G}$ ). According to W. Floyd by rescaling the graph distance $d$ of $\Gamma$ by a scalar function $f: \mathbb{N} \rightarrow \mathbb{R}_{\geq 0}$ one obtains the Cauchy completion $\bar{\Gamma}_{f}$ of the metric space $\left(\Gamma, \delta_{f}\right)$ where $\delta_{f}$ is the rescaled metric. We call this space Floyd completion (see Section 4). The action of $G$ extends continuously to $\bar{\Gamma}_{f}$. By [16] there exists an equivariant continuous map $F$ from the Floyd boundary $\partial_{f} \Gamma=\bar{\Gamma}_{f} \backslash \Gamma$ to the space $T$. The kernel of the map $F$ was described in [17, Theorem A]. Namely if the preimage of a point $p$ is not a single point then $p$ is parabolic and the preimage coincides with the topological boundary of the stabilizer $\operatorname{Stab}_{G} p$ of $p$. We denote by $\partial_{f} \operatorname{Stab}_{G} p$ the Floyd boundary of $\operatorname{Stab}_{G} p$ corresponding to a function $f$.

A subset $X$ of $\Gamma$ is called Floyd ( $r$-)quasiconvex if every Floyd geodesic (with respect to the metric $\delta_{f}$ ) with the endpoints in $X$ belongs to $r$-neighborhood $N_{r}(X)$ for the graph metric $d$ and some $r>0$. In particular if $f$ is the identity then the Floyd quasiconvexity means the standard one. It is well-known that the parabolic subgroups are quasiconvex with respect to $d$ [10] (for another proof see e.g. [17, Corollary 3.9]). Our next Theorem establishes the Floyd quasiconvexity of the parabolic subgroups. 
Theorem C. Let $G$ be a finitely generated group acting 3-discontinuously and 2-cocompactly on a compactum $T$. Let $\Gamma$ be a locally finite, connected graph admitting a cocompact discontinuous action of $G$. Then there exists a Floyd scaling function $f$, such that every parabolic subgroup $H$ of $G$ is Floyd quasiconvex for the Floyd metric $\delta_{f}$.

As a consequence of Theorem $\mathrm{C}$ we obtain the following Corollary which answers our question $[17,1.1]$.

Corollary 7.8. For a scaling function $f$ satisfying conditions (1-3) (see Section 7) one has

$$
F^{-1}(p)=\partial_{f}\left(\operatorname{Stab}_{G} p\right)
$$

for every parabolic point $p \in T$.

Note that it was already known that the map $F$ is 1 -to-1 at conical points [16]. Corollary 7.8 gives a complete description of the preimage of a parabolic point by $F$ as the Floyd boundary of its stabilizer. It gives rise to a complete generalization of the Floyd theorem [12] to the case of relatively hyperbolic groups.

The proof of Theorem C (and Corollary 7.8) in Section 7 and is based on a description of a family of tight curves which are quasigeodesics locally everywhere and geodesic outside the horospheres (see Definition 6.1). Their properties are described in the following Theorem (see Section 6 for more details).

Theorem B. For every tight curve $\gamma$ in the graph of entourages $\mathcal{G}$ there exists a quasigeodesic $\alpha \subset A$ such that every non-horospherical vertex of $\gamma$ belongs to a uniform neighborhood of $\alpha$.

The main step in proving Theorem $\mathrm{C}$ is to show that every Floyd quasigeodesic is tight. We notice that the graph of entourages $\mathcal{G}$ plays here a special role and in the proofs of Theorems $\mathrm{B}$ and $\mathrm{C}$ we deal mainly with it.

This is our second paper in a series of papers about relatively hyperbolic groups. Keeping the same definition of the relative hyperbolicity here we apply however different methods based on the theory of discrete systems of entourages not used in [17].

Acknowledgements. During the work on this paper both authors were partially supported by the ANR grant BLAN 07 - 2183619. We are grateful to the MaxPlanck Institute für Mathematik in Bonn, where a part of the work was done. We also thank the CNRS and the Brasilian-French cooperation grant for support. 
The authors are thankful to Wenyuan Yang for very useful remarks and corrections. We also thank the referee for a careful reading of our paper and providing us a list of corrections.

\section{Convergence groups}

By compactum we mean a compact Hausdorff space. Let $S^{n} T$ denote the quotient of the product space

$$
\underbrace{T \times \ldots \times T}_{n \text { times }}
$$

by the action of the permutation group on $n$ symbols. The elements of $S^{n} T$ are generalized unordered $n$-tuples (i.e. an element may belong to a tuple with some multiplicity). Let $\Theta^{n} T$ be the subset of $S^{n} T$ whose elements are non-ordered $n$-tuples with all distinct components. Put

$$
\Delta^{n} T=S^{n} T \backslash \Theta^{n} T,
$$

the set $\boldsymbol{\Delta}^{2} T$ is just the diagonal of $T^{2}$.

Convention. If the opposite is not stated all group actions on compacta are assumed to have the convergence property.

We refer to [3], [17], [19], [13], and [28] where standard facts related to the convergence groups are proved. We recall below some facts that are used in the paper.

The limit set $\Lambda(G)$ is the set of accumulation (limit) points of the $G$-orbit for the action of $G$ on $T$. It is known that either $|\Lambda(G)| \in\{0,1,2\}$ in which case the action $G \curvearrowright T$ is called elementary or it is a perfect set and the action is not elementary [28].

An elementary action of a group on $T$ is called parabolic if there is unique fixed point called parabolic fixed point.

A limit point $x \in \Lambda(G)$ is called conical if there exists an infinite sequence $g_{n} \in G$ and distinct points $a, b \in T$ such that

$$
g_{n}(y) \rightarrow a \wedge g_{n}(x) \rightarrow b \quad \text { for all } y \in T \backslash\{x\} .
$$

A parabolic fixed point $p \in \Lambda(G)$ is called bounded parabolic if the quotient space $(\Lambda(G) \backslash\{p\}) / \operatorname{Stab}_{G} p$ is compact.

A set $M$ is called $G$-finite if $M / G$ is a finite set. 
An action of a group $G$ on a compactum $T$ is called geometrically finite if every limit point of $T$ is either conical or bounded parabolic. As we have pointed out in the Introduction if $G \curvearrowright T$ is a 3-discontinuous and 2-cocompact action then it is also a geometrically finite one. The opposite statement is also true if one assumes that $T$ is metrizable.

Notation. From now on we fix the notation $\mathcal{P}$ for the set of parabolic points for the 3-discontinuous and 2-cocompact action $G \curvearrowright T$.

\section{Exhaustion of non-finitely generated relatively hyperbolic groups by finitely generated ones}

3.1. Entourages, shadows, betweenness relation. The following definition is motivated by [7, Chapitre 2] and [30].

Definition 3.1. Let $T$ be a compactum. Any (not necessarily open) neighborhood of the diagonal $\Delta^{2} T$ in $S^{2} T$ is called entourage of $T$. The set of all entourages of $T$ is denoted by Ent $T$.

Convention. By definition an entourage consists of non-ordered pairs. However sometimes we identify an entourage $\boldsymbol{e} \in$ Ent $T$ with the symmetric neighborhood $\tilde{\boldsymbol{e}}$ of the diagonal in $T \times T$.

We denote the entourages by bold small characters.

An entourage $\boldsymbol{e}$ determines a graph whose vertex set is $T$, and two vertices $x, y$ are joined by an edge if and only if $\{x, y\} \in \boldsymbol{e}$. Denote by $\Delta_{\boldsymbol{e}}$ the corresponding graph distance which is the maximal distance function with the property $\{x, y\} \in \boldsymbol{e} \Longrightarrow \Delta_{\boldsymbol{e}}(x, y) \leqslant 1$. Note that $\Delta_{\boldsymbol{e}}(x, y)=\infty$ if and only if $x$ and $y$ belong to different connected components of the graph. A set $U \subset T$ is called $\boldsymbol{e}$-small if its $\boldsymbol{e}$-diameter is at most 1 .

The set of all $\boldsymbol{e}$-small sets is denoted by $\operatorname{Small}(\boldsymbol{e})$. For subsets $a, b \subset T$ we define

$$
\Delta_{\boldsymbol{e}}(a, b)=\inf \left\{\Delta_{\boldsymbol{e}}(x, y) \mid x \in a, y \in b\right\}
$$

and

$$
\widetilde{\Delta}_{\boldsymbol{e}}(a, b)=\sup \left\{\Delta_{\boldsymbol{e}}(x, y) \mid x \in a, y \in b\right\} .
$$


From the triangle inequality we have the inequality

$$
\Delta_{e}(a, b) \geq \Delta_{e}(a, c)-\widetilde{\Delta}_{e}(c, b)
$$

frequently used further.

For a subset $a \subset T$ define its $\boldsymbol{e}$-neighborhood $a \boldsymbol{e}$ as $\left\{x \in T \mid \Delta_{\boldsymbol{e}}(x, a) \leqslant 1\right\}$.

For a subset $o$ of $T$ its convex hull in $T \sqcup \operatorname{Ent} T$ is the set

$$
\tilde{o}=o \cup\left\{\boldsymbol{e} \in \operatorname{Ent} T: o^{\prime} \in \operatorname{Small}(\boldsymbol{e})\right\},
$$

where $o^{\prime}$ denotes the complement of $o$.

We equip the space $T \sqcup \mathrm{Ent} T$ with the topology generated by the convex hulls of open subsets of $T$ and the single-point subsets of Ent $T$. Namely a set $w$ in $T \sqcup$ Ent $T$ is declared open if for every point $t \in w \cap T$ there exists and open subset $o$ of $T$ such that $t \in o$ and $\tilde{o} \subset w$. In particular Ent $T$ is a discrete open subset and $T$ is a closed subspace of $T \sqcup \mathrm{Ent} T$.

Example 1. The definition of the topology on $T \sqcup$ Ent $T$ can be illustrated in terms of the open subsets of the compactified real hyperbolic space $\mathbb{H}^{n} \cup \partial_{\infty} \mathbb{H}^{n}$. Let $B$ be a bounded subset of $\mathrm{H}^{n}$. Define an entourage $\boldsymbol{e}_{B} \in \operatorname{Ent}\left(\partial \mathrm{H}^{n}\right)$ in the following way: $\{x, y\} \in \boldsymbol{e}_{B}$ if and only if the geodesic $\gamma(x, y)$ with the endpoints $x$ and $y$ misses $B$. So a set $o \subset T=\partial_{\infty} \mathbb{H}^{n}$ is $\boldsymbol{e}_{B}$-small if and only if $B$ is contained in the convex hull of $o$ in $\mathrm{H}^{n}$ (see Figure 1). Thus $B$ is close to $a$ in the topology of $\mathbb{H}^{n} \cup \partial_{\infty} \mathbb{H}^{n}$ if and only if $\boldsymbol{e}_{B}$ is close to $a$ in the topology of $T \sqcup$ Ent $T$. By the above definition $\tilde{o}$ is obtained by adding to $o$ every entourage for which $o^{\prime}$ is small.

Definition 3.2 ([15]). Two entourages $\boldsymbol{a}$ and $\boldsymbol{b}$ are said to be unlinked if there exist $a \in \operatorname{Small}(\boldsymbol{a})$ and $b \in \operatorname{Small}(\boldsymbol{b})$ such that $T=a \cup b$. We denote this relation by $\boldsymbol{a} \bowtie \boldsymbol{b}$. In the opposite case we say that $\boldsymbol{a}$ and $\boldsymbol{b}$ are linked, and write $\boldsymbol{a} \# \boldsymbol{b}$.

Denote by La the set $\{\boldsymbol{b} \in$ Ent $T \mid \boldsymbol{a} \# \boldsymbol{b}\}$. It is enough for our purposes to consider only sufficiently small entourages implying the following.

Convention. All considered entourages are supposed to be self-linked:

$$
\boldsymbol{a} \in \operatorname{Ent} T: \boldsymbol{a} \# \boldsymbol{a} \text {. }
$$




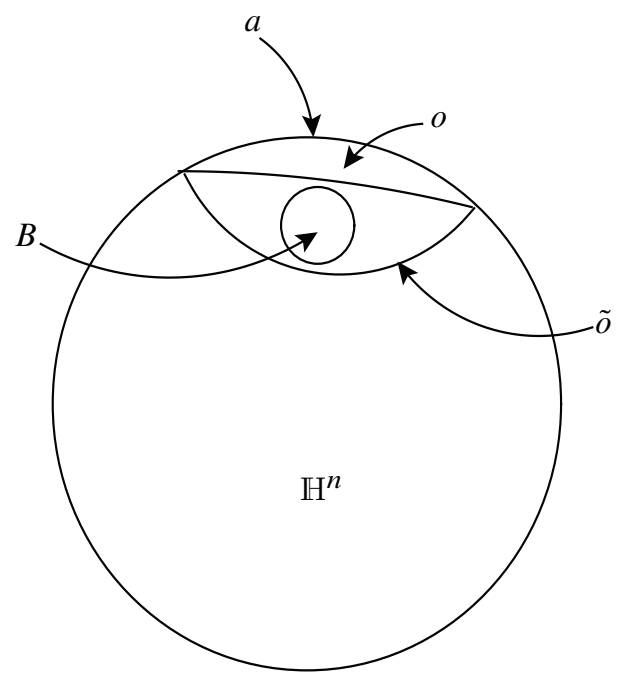

Figure 1. Bounded set in $\mathrm{H}^{n}$ and its visibility entourage.

Definition 3.3 ([15]). Let $\boldsymbol{a}$ and $\boldsymbol{b}$ be two unlinked entourages. We define the following "shadow" sets:

$$
\operatorname{Sh}_{\boldsymbol{a}} \boldsymbol{b}=\left\{a \in \operatorname{Small}(\boldsymbol{a}) \mid a^{\prime} \in \operatorname{Small}(\boldsymbol{b})\right\}
$$

and

$$
\operatorname{sh}_{\boldsymbol{a}} \boldsymbol{b}=\bigcap \mathrm{Sh}_{\boldsymbol{a}} \boldsymbol{b}=\left(\bigcup \mathrm{Sh}_{\boldsymbol{b}} \boldsymbol{a}\right)^{\prime}
$$

It is shown in [15, Lemma S0] that if $\boldsymbol{a} \bowtie \boldsymbol{b}$ and $\operatorname{diam}_{\boldsymbol{a}} T>2$ then $\operatorname{sh}_{\boldsymbol{a}} \boldsymbol{b} \neq \varnothing$; and if $\operatorname{diam}_{\boldsymbol{a}} T>4$ then $\operatorname{sh}_{\boldsymbol{a}} \boldsymbol{b}$ has a nonempty interior.

Convention. We consider only the entourages $\boldsymbol{a}$ with $\operatorname{diam}_{\boldsymbol{a}} T>4$. So every shadow has non-empty interior.

Example 2. Using the notations of Example 1 let $\boldsymbol{a}=\boldsymbol{e}_{A}$ and $\boldsymbol{b}=\boldsymbol{e}_{B}$ for two disjoint balls $A$ and $B$ in the hyperbolic space $\mathbb{H}^{n}$. Then the shadow $\operatorname{sh}_{\boldsymbol{a}} \boldsymbol{b}$ is given by the intersection with $\partial \mathrm{H}^{n}$ of the boundaries of all hyperbolic half-spaces of $\mathrm{H}^{n}$ containing $B$ and not containing $A$ and similarly for $\operatorname{sh}_{b} a$ (see Figure 2). 


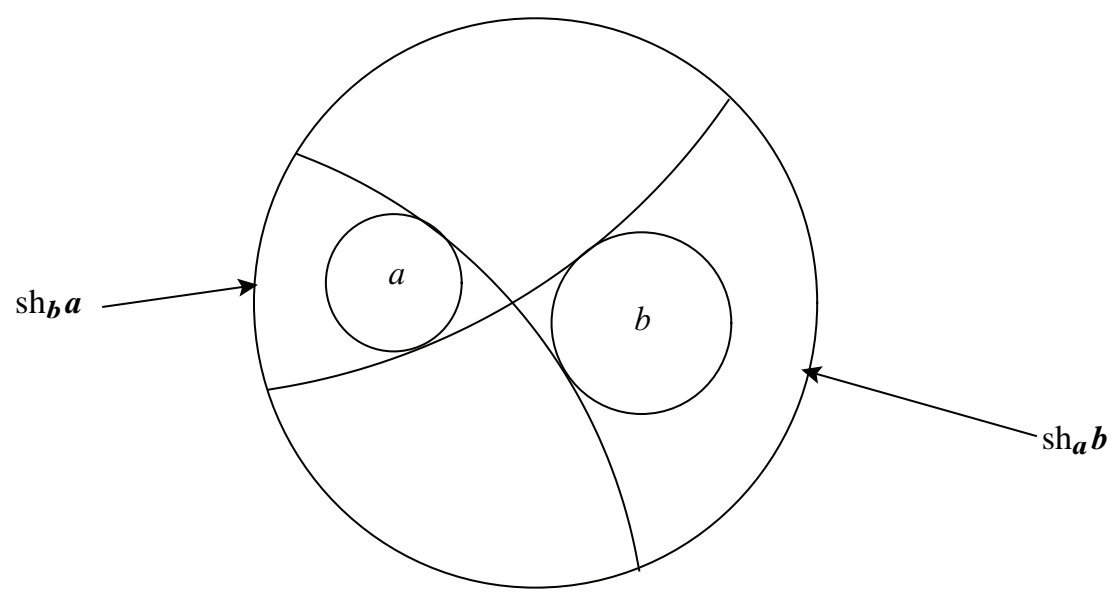

Figure 2. Shadows $\operatorname{sh}_{\boldsymbol{b}} \boldsymbol{a}$ and $\operatorname{sh}_{\boldsymbol{b}} \boldsymbol{a}$.

Definition 3.4 (Betweenness relation). Let $k$ be a positive integer.

1) Suppose $\boldsymbol{a}, \boldsymbol{b}, \boldsymbol{c} \in$ Ent $T$. We say that an entourage $\boldsymbol{b}$ lies between (or $k$-between) $\boldsymbol{a}$ and $\boldsymbol{c}$, and write $\boldsymbol{a}-\boldsymbol{b}-\boldsymbol{c}(k)$ (or simply $\boldsymbol{a}-\boldsymbol{b}-\boldsymbol{c}$ ), if $\boldsymbol{a} \bowtie \boldsymbol{b} \bowtie \boldsymbol{c}$ and $\Delta_{\boldsymbol{b}}\left(\operatorname{sh}_{\boldsymbol{b}} \boldsymbol{a}, \operatorname{sh}_{\boldsymbol{b}} \boldsymbol{c}\right)>k$.

2) Let $\boldsymbol{a}, \boldsymbol{b} \in \operatorname{Ent} T$ and let $p \in T$. We say that $\boldsymbol{b}$ lies between (or $k$-between) $\boldsymbol{a}$ and $p$ if $\boldsymbol{a} \bowtie \boldsymbol{b}$ and $\Delta_{\boldsymbol{b}}\left(\operatorname{sh}_{\boldsymbol{b}} \boldsymbol{a}, b\right)>k$ for any $\boldsymbol{b}$-small neighborhood $b$ of $p$

We write $\boldsymbol{a}-\boldsymbol{b}-p(k)$ (or simply $\boldsymbol{a}-\boldsymbol{b}-p$ ) in this case.

3) Let $\boldsymbol{b} \in \operatorname{Ent} T$ and let $p, q \in T$ be two distinct points. We say that $\boldsymbol{b}$ lies between (or $k$-between) $p$ and $q$, and write $q-\boldsymbol{b}-p$ ( $k$ ) (or simply $q-\boldsymbol{b}-p$ ), if $\Delta_{\boldsymbol{b}}\left(b_{1}, b_{2}\right)>k$ for any $\boldsymbol{b}$-small neighborhoods $b_{1}$ and $b_{2}$ of the points $p$ and $q$ respectively.

Remarks 3.5. a) The betweenness relations 2) and 3) represent an extension "by continuity" of the relation 1) between entourages to the points of $T$. Note that the middle object in the relation $\boldsymbol{a}-\boldsymbol{b}-\boldsymbol{c}$ is always an entourage.

Note also that if $\Delta_{\boldsymbol{b}}\left(\mathrm{sh}_{\boldsymbol{b}} \boldsymbol{a}, b_{0}\right)>k$ for some $\boldsymbol{b}$-small neighborhood $b_{0}$ of $p$ then for any such $b$ we have $\Delta_{\boldsymbol{b}}\left(\operatorname{sh}_{\boldsymbol{b}} \boldsymbol{a}, b\right) \geq \Delta_{\boldsymbol{b}}\left(\operatorname{sh}_{\boldsymbol{b}} \boldsymbol{a}, b_{0}\right)-\Delta_{\boldsymbol{b}}\left(b, b_{0}\right)>k-2$ as $p \in b \cap b_{0}$ and $\widetilde{\Delta}_{\boldsymbol{b}}\left(b, b_{0}\right) \leq 2$. Therefore we will always assume further that $k>2$.

b) Definition 3.4 in cases 2) and 3) differs from the corresponding definition in [15] where the condition $\Delta_{\boldsymbol{b}}\left(\operatorname{sh}_{\boldsymbol{a}} \boldsymbol{b}, p\right)>k$ is stated instead of 2). The above betweenness definition is stronger than that of [15] and so is easier to use. However both of them are quite close: the $k$-betweenness 2) implies $k$-betweenness 
of [15]. On the other hand since the diameter of any small neighborhood is less than 1 the $k+1$-betweenness of [15] implies (by the triangle inequality) the $k$-betweenness 2). We will use results of [15] keeping in mind this relation.

Lemma 3.6 (continuity property). Suppose that $\boldsymbol{a}-\boldsymbol{c}-p(k)(k \in \mathbb{N})$ where $\boldsymbol{a} \in T \sqcup$ Ent $T, \boldsymbol{c} \in$ Ent $T, p \in T$. Suppose that $p \in T$ is an accumulation point for an infinite subset $B$ of Ent $T$. Then there exists $\boldsymbol{b} \in B$ such that $\boldsymbol{a}-\boldsymbol{c}-\boldsymbol{b}(k)$.

Proof. Let first $\boldsymbol{a} \in$ Ent $T$ be an entourage. Let $c=U_{p}$ be an open $\boldsymbol{c}$-small set containing $p$ such that $\Delta_{c}\left(c, \operatorname{sh}_{\boldsymbol{c}} \boldsymbol{a}\right)>k$. By definition of the topology of $T \sqcup$ Ent $T$ the complement $c^{\prime}$ is $\boldsymbol{b}$-small for some $\boldsymbol{b} \in B$. Then $c^{\prime} \subset \bigcup \mathrm{Sh}_{\boldsymbol{b}} \boldsymbol{c}$, and $c \supset \operatorname{sh}_{c} b=\left(\bigcup \operatorname{Sh}_{b} c\right)^{\prime}$. Thus $\Delta_{c}\left(\operatorname{sh}_{c} \boldsymbol{a}, \operatorname{sh}_{\boldsymbol{c}} \boldsymbol{b}\right)>\Delta_{\boldsymbol{c}}\left(\mathrm{sh}_{\boldsymbol{c}} \boldsymbol{a}, c\right)>k$.

If now $a \in T$ then for a $c$-small neighborhood $U$ containing $a$, we obtain similarly $\Delta_{\boldsymbol{c}}\left(U, \operatorname{sh}_{\boldsymbol{c}} \boldsymbol{b}\right)>\Delta_{\boldsymbol{c}}\left(U, U_{p}\right)>k$. So we still have $a-\boldsymbol{c}-\boldsymbol{b}(k)$ for $\boldsymbol{b} \in B$.

Definition 3.7 (tubes). [15] A sequence $P$ of elements $\boldsymbol{a}_{n}$ of $T \sqcup$ Ent $T$ is called $k$-tube (or tube) if, for all $n$,

$$
\left(\boldsymbol{a}_{n} \bowtie \boldsymbol{a}_{n+1}\right) \wedge\left(\boldsymbol{a}_{n-1}-\boldsymbol{a}_{n}-\boldsymbol{a}_{n+1}(k)\right)
$$

whenever $\boldsymbol{a}_{n \pm 1}$ are defined.

Lemma 3.8. 1) OR DER ING. For any three entourages at most one can be between the others.

2) ConveXity. If $\boldsymbol{a}-\boldsymbol{b}-\boldsymbol{c}(4)$ and $\boldsymbol{a}, \boldsymbol{c} \in \mathrm{L} \boldsymbol{d}$ then $\boldsymbol{b} \in \mathrm{L} \boldsymbol{d}$.

Proof. 1) Indeed if not, we obtain $\boldsymbol{a}-\boldsymbol{b}-\boldsymbol{c}$ and $\boldsymbol{a}-\boldsymbol{c}-\boldsymbol{b}$ for some $\boldsymbol{a}, \boldsymbol{b}, \boldsymbol{c}$. The transitivity of the betweenness relation [15] would imply $\boldsymbol{a}-\boldsymbol{b}-\boldsymbol{a}$ and so $\boldsymbol{a} \bowtie \boldsymbol{a}$ which is impossible by our convention (1).

2) Otherwise $\boldsymbol{b} \bowtie \boldsymbol{d}$ and we have $T=b \cup d=a \cup b_{1}=c \cup b_{2}$ where $b_{i}, b \in \operatorname{Small}(\boldsymbol{b})(i=1,2), d \in \operatorname{Small}(\boldsymbol{d}), a \in \operatorname{Small}(\boldsymbol{a}), c \in \operatorname{Small}(\boldsymbol{c})$. It follows that $b \cap b_{1}=\emptyset$ or $b \cap b_{2}=\emptyset$ since otherwise $\Delta_{\boldsymbol{b}}\left(b_{1}, b_{2}\right) \leq 2$ and we would have $\Delta_{\boldsymbol{b}}\left(\operatorname{sh}_{\boldsymbol{b}} \boldsymbol{a}, \mathrm{sh}_{\boldsymbol{b}} \boldsymbol{c}\right) \leq 2+\widetilde{\Delta}_{\boldsymbol{b}}\left(\operatorname{sh}_{\boldsymbol{b}} \boldsymbol{a}, b_{1}\right)+\widetilde{\Delta}_{\boldsymbol{b}}\left(\operatorname{sh}_{\boldsymbol{b}} \boldsymbol{c}, b_{2}\right) \leq 4\left(\operatorname{assh}_{\boldsymbol{b}} \boldsymbol{a} \subset b_{1}\right.$ and $\operatorname{sh}_{\boldsymbol{b}} c \subset b_{2}$ ) which is impossible. If, for instance, $b \cap b_{1}=\emptyset$ then $b_{1} \subset d$ and $\boldsymbol{a} \bowtie \boldsymbol{d}$. A contradiction. 
3.2. Discrete sets of entourages. Horospheres. Until the end of Section 3 we fix a 3-discontinuous 2-cocompact action $G \curvearrowright T$ of a group $G$ on a compactum $T$.

Definition 3.9. A set $A$ of entourages on $T$ is called discrete if

$$
|\{\boldsymbol{a} \in A: \boldsymbol{a} \# \boldsymbol{w}\}|<\infty \quad \text { for all } \boldsymbol{w} \in \operatorname{Ent} T \text {. }
$$

By [15, Proposition P] the set $\{g \in G: g \boldsymbol{a} \# \boldsymbol{w}\}$ is finite for all $\boldsymbol{w}, \boldsymbol{a} \in$ Ent $T$. This property is called Dynkin property [14]. Hence every $G$-finite set is discrete.

Let $A \subset$ Ent $T$ be a $G$-finite set of entourages. Denote by $\widetilde{T}$ the subspace $T \sqcup A$ of $T \sqcup$ Ent $T$. Since $A$ is discrete $\widetilde{T}$ is compact [15, Proposition D].

Definition 3.10. Let $\mathcal{G}=\mathcal{G}_{A}$ be the graph whose vertex set $\mathcal{G}^{0}$ is $A$ and the edge set $\mathcal{G}^{1}$ is the set of pairs $\{\boldsymbol{a}, \boldsymbol{b}\}$ such that $\boldsymbol{a} \# \boldsymbol{b}$. Denote by $d_{A}$ the corresponding graph distance.

Since $G$ acts on $T$ by homeomorphisms it acts isometrically on $\left(\mathcal{G}, d_{A}\right)$.

Lemma 3.11. The group $G$ is finitely generated if and only if there exists a connected graph $\mathcal{G}_{A}$.

Proof. Suppose first that $G$ admits a finite set of generators $S$ (id $\in S$ ). Since $A$ is $G$-finite we have $A=\bigcup_{i=1}^{l} G\left(\boldsymbol{a}_{i}\right)$. Any entourage $\boldsymbol{a}_{i}$ contains a sub-entourage $\boldsymbol{a}_{i}^{\prime}$ such that

$$
\boldsymbol{a}_{i}^{\prime} \# s \boldsymbol{a}_{j}^{\prime} \quad(i, j \in\{1, \ldots, l\}) \text { for all } s \in S .
$$

So up to choosing the entourages $\boldsymbol{a}_{i}(i=1, \ldots, l)$ to be sufficiently small we can assume that the above property is satisfied. Then all vertices in the set $\bigcup_{i} S a_{i}$ are pairwise connected by edges. For any vertex $\boldsymbol{v} \in \mathcal{G}_{A}$ there exists $i \in\{1, \ldots, l\}$ and $g \in G$ such that $\boldsymbol{v}=g\left(\boldsymbol{a}_{i}\right)$ and $g=s_{i_{1}} s_{i_{2}} \ldots s_{i_{k}}\left(s_{i_{j}} \in S\right)$. Then $\mathcal{G}_{A}$ contains the edges $e=\left(s_{i_{k}}\left(\boldsymbol{a}_{i}\right), \boldsymbol{a}_{i}\right), e^{\prime}=\left(s_{i_{k-1}}\left(\boldsymbol{a}_{i}\right), \boldsymbol{a}_{i}\right)$, and so the path $s_{i_{k-1}} e \cup e^{\prime}$ between $\boldsymbol{a}_{i}$ and $s_{i_{k-1}} s_{i_{k}}\left(\boldsymbol{a}_{i}\right)$. Continuing in this way we obtain a path between $\boldsymbol{v}$ and $\boldsymbol{a}_{i}$.

Conversely suppose that $\mathcal{G}_{A}$ is connected. Let $S$ be the set $\left\{s \in G \mid s \boldsymbol{a}_{j} \# \boldsymbol{a}_{i}\right.$, $1 \leq i \leq l\}$ where $A=\bigcup_{i=1}^{l} G \boldsymbol{a}_{i}$. By Dynkin property the set $S$ is finite. For any $g \in G$ there is a path $l=\left\{\boldsymbol{a}_{i}, \boldsymbol{b}_{2}, \ldots, \boldsymbol{b}_{n-1}, \boldsymbol{a}\right\} \subset \mathcal{G}_{A}$ between the vertices $\boldsymbol{a}=g\left(\boldsymbol{a}_{i}\right)$ and $\boldsymbol{a}_{i}$. Then $\boldsymbol{b}_{2} \# \boldsymbol{a}_{i}$ so there exists $s_{1} \in S$ such that $\boldsymbol{b}_{2}=s_{1}\left(\boldsymbol{a}_{j}\right)$ $(1 \leq j \leq l)$. Thus $s_{1}^{-1} \boldsymbol{b}_{3} \# \boldsymbol{a}_{j}$ and there exists $s_{2} \in S$ such that $\boldsymbol{b}_{3}=s_{1} s_{2} \boldsymbol{a}_{k}$ $(1 \leq k \leq l)$. Continuing in this way we obtain $\boldsymbol{a}=s_{1} s_{2} \ldots s_{n} \boldsymbol{a}_{r}(1 \leq r \leq l)$. Then $g^{-1} s_{1} s_{2} \ldots s_{n}\left(\boldsymbol{a}_{r}\right)=\boldsymbol{a}_{i}$ and so $g^{-1} s_{1} s_{2} \ldots s_{n}$ belongs to $S$ (by (1) of Section 3.1). The lemma is proved. 
It follows from Dynkin property and our convention (1) that the stabilizer of each edge and each vertex of $\mathcal{G}$ is finite. The action $G \curvearrowright T$ is 2-cocompact so by [15, Proposition E] we can suppose that the set $A$ is a single orbit $G\left(\boldsymbol{a}_{0}\right)$ $\left(a_{0} \in\right.$ Ent $\left.T\right)$ having the following properties.

i) $m$-SEPARATION PRoperty. For all $(p, q) \in \Theta^{2} T$ there exists $\boldsymbol{a} \in A$ such that

$$
p-\boldsymbol{a}-q(m)
$$

for a fixed $m \in \mathbb{N}$.

ii) generating Property. For all $\boldsymbol{u} \in$ Ent $T$ there exists $\boldsymbol{a}_{i} \in A, \mathrm{i}=1, \ldots, 1$, such that

$$
\boldsymbol{u} \supset \bigcap_{i=1}^{l} \boldsymbol{a}_{i},
$$

i.e. $A$ generates Ent $T$ as a filter.

Convention 3.12. From now on we fix an unlinked entourage $\boldsymbol{a}_{0} \in \operatorname{Ent} T$ (see (1) of Section 3.1) such that its orbit $A=G\left(\boldsymbol{a}_{0}\right)$ satisfies $m$-separating and generating properties. The value of $m$ can be easily restored in each statement. Keeping in mind that this value might be needed to be increased further we just suppose that $m$ is sufficiently large.

Furthermore if $G$ is finitely generated we will always assume (by Lemma 3.11) that the graph $\mathcal{G}$ is connected.

Remark. The graph $\mathcal{G}$ plays the role of the Cayley graph $\mathrm{C} a(G)$ if $G$ is finitely generated, however by Dynkin property it is always a locally finite graph. The space $\widetilde{T}=T \sqcup A$ is a compactification of $A=\mathcal{G}^{0}$ similar to the Floyd completion (see Section 4). Every action $G \curvearrowright T$ can be naturally extended to the space $\widetilde{T}$.

Lemma 3.13. The space $\widetilde{T}=T \sqcup A$ is a compactum.

Proof. The space $T$ is Hausdorff. To prove that $\widetilde{T}$ is Hausdorff we will consider three different cases. Let first $x, y$ be distinct points of $T$ then there exist disjoint closed neighborhoods $U_{x}$ and $U_{y}$ in $T$. Their convex hulls $\widetilde{U}_{x}=U_{x} \cup\left\{\boldsymbol{e} \in A: U_{x}^{\prime} \in\right.$ Small $(\boldsymbol{e})\}$ and $\tilde{U}_{y}=U_{y} \cup\left\{\boldsymbol{d} \in A: U_{y}^{\prime} \in \operatorname{Small}(\boldsymbol{d})\right\}$ are neighborhoods of these points in the topology of $\widetilde{T}$ induced from $T \sqcup$ Ent $T$ (see ( $\dagger$ ) of Section 3.1). If $\boldsymbol{a} \in A \cap \widetilde{U}_{x} \cap \widetilde{U}_{y}$ then $U_{x}^{\prime}$ and $\widetilde{U}_{y}^{\prime}$ are both $\boldsymbol{a}$-small. Since $U_{x}$ and $U_{y}$ are disjoint we have $U_{x}^{\prime} \cup U_{y}^{\prime}=T$ and so $\boldsymbol{a} \# \boldsymbol{a}$ contradicting our convention (1) of Section 3.1. Hence $\tilde{U}_{x} \cap \tilde{U}_{y}=\emptyset$ in this case. 
If now $\boldsymbol{x} \in A$ and $y \in T$ then by the same reason any $\boldsymbol{x}$-small neighborhood of $y$ in $\widetilde{T}$ cannot contain $\boldsymbol{x}$. Since every entourage is open in $\widetilde{T}$ we are done in this case too. If finally both points are entourages they coincide with their disjoint neighborhoods. So $\widetilde{T}$ is Hausdorff.

The compactness of $\widetilde{T}$ follows from [15, Proposition D].

Proposition 3.14. If a group $G$ acts 3-discontinuously on a compactum $T$ then the induced action on $\widetilde{T}=T \cup A$ is also 3-discontinuous.

Remark. In [16, Theorem 5.1] it is proved that there is a unique topology on the compactified space $\widetilde{T}$ with respect to which the action is 3-discontinuous. The argument below provides a simple proof of this for the induced topology on $\tilde{T} \subset T \sqcup$ Ent $T$ introduced above.

Proof. For a subset $X \subset T$ denote by $\tilde{X}=X \cup\left\{\boldsymbol{a} \in A \mid X^{\prime} \in \operatorname{Small}(\boldsymbol{a})\right\} \subset \widetilde{T}$ its convex hull in $\widetilde{T}$. In case if $X=\{\boldsymbol{a}\}$ where $\boldsymbol{a} \in A$ is an entourage we put $\tilde{X}=\boldsymbol{a}$. For every $g \in G$ denote by $\tilde{g}$ its natural extension to $\tilde{T}$.

Every point $x \in \Theta^{3} \widetilde{T}$ admits a closed neighborhood which is a "cube" $\widetilde{K}=\tilde{X} \times \tilde{Y} \times \widetilde{Z}$ where $X, Y$ and $Z$ are either disjoint closed subsets of $T$ or some of $\tilde{X}, \tilde{Y}, \widetilde{Z}$ are isolated entourages (in the latter case we call the corresponding cube degenerate). Every compact subset of $\Theta^{3} \widetilde{T}$ is a finite union of such cubes. So it is enough to prove that for two cubes $\widetilde{K}_{i}=\widetilde{X}_{i} \times \tilde{Y}_{i} \times \widetilde{Z}_{i} \subset \Theta^{3} \widetilde{T}(i=0,1)$ the following set is finite:

$$
S=\left\{g \in G \mid \widetilde{g} \tilde{X}_{0} \cap \tilde{X}_{1} \neq \emptyset, \tilde{g} \tilde{Y}_{0} \cap \tilde{Y}_{1} \neq \emptyset, \tilde{g} \widetilde{Z}_{0} \cap \widetilde{Z}_{1} \neq \emptyset\right\} .
$$

Suppose to the contrary that $S$ is infinite. Since the action $G \curvearrowright T$ is 3-discontinuous, every accumulation point of $S$ with respect to Vietoris topology is a cross

$$
\langle p, q\rangle^{\times}=p \times T \sqcup T \times q,
$$

cf. [15, Proposition P]. Consider now all possible cases.

CASE 1 . Both cubes are not degenerate, i.e. $X_{i}, Y_{i}, Z_{i}(i=0,1)$ are all closed disjoint subsets of $T$.

Note that at least one of the "squares" $X_{0} \times X_{1}, Y_{0} \times Y_{1}$ or $Z_{0} \times Z_{1}$ does not meet the cross. Indeed otherwise two of them intersect both either $p \times T$ or $T \times q$ which is impossible as $X_{i}, Y_{i}$ and $Z_{i}$ are pairwise disjoint for $i \in\{0,1\}$. 
Let us assume that e.g. $Z_{0} \times Z_{1} \cap\langle p, q\rangle^{\times}=\emptyset$. Let $g \in S$ be a homeomorphism whose graph is contained in the neighborhood $T^{2} \backslash Z_{0} \times Z_{1}$ of $\langle p, q\rangle^{\times}$. Then $g Z_{0} \cap Z_{1}=\emptyset$. However $\tilde{g} \widetilde{Z}_{0} \cap \widetilde{Z}_{1} \neq \emptyset$. So there exists $a \in \widetilde{Z}_{0} \backslash Z_{0}$ such that $\tilde{g} \boldsymbol{a} \in \widetilde{Z}_{1}$. By definition of the convex hull $Z_{0}^{\prime}$ and $\left(g^{-1}\left(Z_{1}\right)\right)^{\prime}$ are $\boldsymbol{a}$-small. Since $\left(g^{-1} Z_{1}\right)^{\prime} \cup Z_{0}^{\prime}=T$ we obtain that $T$ is the union of two $\boldsymbol{a}$-small sets, so $\boldsymbol{a} \# \boldsymbol{a}$ contradicting our Convention 3.12.

CASE 2. At least one of the cubes is degenerate.

Then some of the sets $\tilde{X}_{i}, \tilde{Y}_{i}, \widetilde{Z}_{i}$ are entourages. Note that since $g \tilde{X}_{0} \cap \tilde{X}_{1} \neq \emptyset$ for infinitely many $g \in S$, by Dynkin property $\tilde{X}_{0}$ and $\tilde{X}_{1}$ cannot be entourages simultaneously. The same is true for $\tilde{Y}_{i}$ and $Z_{i}(i=0,1)$. So there could be at most 3 entourages among these 6 sets. We consider all the possibilities below.

SubCASE 2.1. There is only one degenerate cube.

We can assume that $\tilde{X}_{0}=\boldsymbol{a}$ for some $\boldsymbol{a} \in A$. Then for all $g \in S$ we have $g \boldsymbol{a} \in \tilde{X}_{1}$. So $g^{-1} X_{1}^{\prime}$ is $\boldsymbol{a}$-small. For a limit cross $<p, q>^{\times}$for the set $S$ and $\boldsymbol{a}$-small neighborhoods $U_{p}$ and $U_{q}$ of the points $p$ and $q$ respectively there exists $g \in S$ such that $g U_{p}^{\prime} \subset U_{q}$ or $g^{-1} U_{q}^{\prime} \subset U_{p}$. If now $U_{q} \cap X_{1}=\emptyset$ then $T$ would be the union of $\boldsymbol{a}$-small sets $g^{-1} X_{1}^{\prime}$ and $g^{-1} U_{q}^{\prime}$ contradicting the unlinkness condition $\boldsymbol{a} \# \boldsymbol{a}$. So for every $\boldsymbol{a}$-small neighborhood $U_{q}$ of $q$ we have $U_{q} \cap X_{1} \neq \emptyset$. Since $X_{1}$ is closed it follows that $q \in X_{1}$.

At most one of the disjoint sets $Y_{0}$ or $Z_{0}$ can contain the other point $p$ of the cross, let $p \notin Z_{0}$. Then for any neighborhood $U_{q}$ and for infinitely many elements $g \in S$ we have $g Z_{0} \subset U_{q}$. If $g Z_{0} \cap Z_{1} \neq \emptyset$ for infinitely many $g \in S$ then $q$ is an accumulation point for $Z_{1}$, and since $Z_{1}$ is closed we obtain that $q \in Z_{1} \cap X_{1}$ which is impossible. So for almost all $g \in S: g Z_{0} \cap Z_{1}=\emptyset$ and this situation has been excluded in Case 1 .

SubCase 2.2. There are two degenerate cubes.

Note that they cannot belong to the same level, namely if $\tilde{X}_{0}=\boldsymbol{a} \in A$ and $\tilde{Y}_{0}=\boldsymbol{b} \in A$ then by the argument of Subcase 2.1 we must have $q \in Y_{1} \cap X_{1}$ which is impossible.

So let $\widetilde{Y}_{1}=\boldsymbol{b} \in A$ and $\tilde{X}_{0}=\boldsymbol{a} \in A$. By the argument of Subcase 2.1 applied now to the inverse elements of $S$ we obtain that $p \in Y_{0}$. Hence for almost all elements $g \in S$ we still have $g Z_{0} \cap Z_{1}=\emptyset$ which is impossible by Case 1 . 
SubCASe 2.3. There are three degenerate cubes.

Then there are at least two of three entourages which are among of the sets of the same level: $\widetilde{X}_{i}, \widetilde{Y}_{i}, \widetilde{Z}_{i}(i=0$ or $i=1)$ which is impossible. So neither case can happen. The proposition is proved.

Lemma 3.15. Let $B$ be an infinite subset of $A$ and $C=N_{d}(B)$ where $N_{d}(B)$ is a $d$-neighborhood of $B$ in $\widetilde{T}$. Then the topological boundaries of $B$ and $C$ coincide.

In particular, if $\left(\boldsymbol{b}_{n}\right)_{n}$ and $\left(\boldsymbol{c}_{n}\right)_{n}$ are two sequences in $A$ such that $d_{A}\left(\boldsymbol{b}_{n}, \boldsymbol{c}_{n}\right)$ is uniformly bounded, then $\left(\boldsymbol{b}_{n}\right)_{n}$ converges to a point $p \in T$ if and only if $\boldsymbol{c}_{n} \rightarrow p$.

Proof. The second claim directly follows from the first one. So to prove the lemma we need only to show that every accumulation point of $C$ is also an accumulation point of $B$. Suppose not and there exists a point $r \in \partial C \backslash \partial B$. Then for every neighborhood $U_{r}$ of $r$ in $\widetilde{T}$ there exists an infinite subset $C_{0} \subset C$ such that for all $c \in C_{0}$ we have $c \in U_{r}$ implying that $U_{r}^{\prime} \subset c$ for some $c \in \operatorname{Small}(c)$.

Arguing by induction on $d$ without loss of generality we may assume that $d=1$. So for all $\boldsymbol{c} \in C$ there exists $\boldsymbol{b} \in B$ such that $\boldsymbol{c} \# \boldsymbol{b}$. Then there exists a subset $B_{0} \subset B$ such that $d_{A}\left(B_{0}, C_{0}\right) \leq 1$. Since $C_{0}$ is infinite by discreteness of $A$ the set $B_{0}$ is infinite too. Let $p \in T \backslash\{r\}$ be an accumulation point of $B_{0}$. Then for every neighborhood $U_{p}$ of $p$ there exists $\boldsymbol{b} \in B_{0}$, corresponding to some $\boldsymbol{c} \in C_{0}$, for which $U_{p}^{\prime} \subset b$ where $b \in \operatorname{Small}(\boldsymbol{b})$. Choosing $U_{p}$ to be disjoint from $U_{r}$ we obtain $b \cup c=T$ and so $\boldsymbol{b} \bowtie \boldsymbol{c}$. A contradiction.

Definition 3.16 (horospheres, conical and parabolic points [15]). Let $k$ be a fixed positive integer, and let $A$ be the above discrete set of entourages.

1) We say that a point $p \in T$ and an entourage $\boldsymbol{e}$ are neighbors (with respect to $A$ ) and write $\underset{A, k}{\#} p$, if there is no $\boldsymbol{a} \in A$ such that $\boldsymbol{e}-\boldsymbol{a}-p(k)$.

2) The horosphere $T_{A, k}(p)$ (or $T_{k}(p)$ or $T(p)$ ) at the point $p \in T$ is the set

$$
T_{A, k}(p)=\{\boldsymbol{e} \in A \mid \boldsymbol{e} \underset{A, k}{\#} p\} .
$$

3) A point $x \in T$ is called (A,k)-conical (or just conical) if $T_{A, k}(x)=\emptyset$.

4) A point $p \in T$ is called (A,k)-parabolic (or just parabolic) if $T_{A, k}(p)$ is infinite.

It is shown in [15] that the notions of $(A, k)$-conical and $(A, k)$-parabolic points for $k \geq 3$ (see also Remarks 3.5) are equivalent to the standard definitions (see Section 2) of conical and bounded parabolic points respectively. 
Lemma 3.17 ([15]). If the action $G \curvearrowright T$ is 3-discontinuous and 2-cocompact then every limit point of this action is either conical or bounded parabolic. Furthermore the set of non-conical points is $G$-finite and for every parabolic point $p \in T$ the set $T(p)$ is $\operatorname{Stab}_{G} p$-finite.

The next lemma is proved in [15, Lemma P2] for closed entourages. We prove it below in a general form.

Lemma 3.18. For every $d>0$ the parabolic point $p$ is the unique accumulation point of the d-neighborhood $N_{d}\left(T_{A, k}(p)\right)$ of the horosphere $T_{A, k}(p)$.

Proof. By Lemma 3.15 it is enough to prove the statement for the horosphere $T_{A, k}(p)$. Suppose it admits two distinct accumulation points $p$ and $q$. Since the set $A$ is $m$-separating there exists $\boldsymbol{a} \in A$ such that $p-\boldsymbol{a}-q(k)$ for some $k \leq m$. Then by Lemma 3.6 there exists $\boldsymbol{b} \in T_{\boldsymbol{A}, k}(p)$ such that $p-\boldsymbol{a}-\boldsymbol{b}(k)$ which is not possible.

We have the following transitivity property:

Lemma 3.19. If $\boldsymbol{a}, \boldsymbol{b}, \boldsymbol{c} \in \operatorname{Ent} T, p \in T$ and $k>2$. Then $\boldsymbol{a}-\boldsymbol{b}-p(k)$ and $\boldsymbol{b}-\boldsymbol{c}-p(k)$ imply $\boldsymbol{a}-\boldsymbol{c}-p(k)$.

Proof. If $a \in \mathrm{Sh}_{\boldsymbol{a}} \boldsymbol{b}$ and $c \in \mathrm{Sh}_{\boldsymbol{c}} \boldsymbol{b}$, then the sets $b=a^{\prime}, b_{1}=c^{\prime}$ are $\boldsymbol{b}$-small and $a \cup b=b_{1} \cup c=T$. Then for a $c$-small neighborhood $c_{0}$ of $p$ we have $\Delta_{c}\left(c, c_{0}\right) \geq$ $\Delta_{\boldsymbol{c}}\left(\operatorname{sh}_{\boldsymbol{c}} \boldsymbol{b}, c_{0}\right)-\widetilde{\Delta}_{\boldsymbol{c}}\left(\operatorname{sh}_{\boldsymbol{c}} \boldsymbol{b}, c\right)>k-1$. So $\Delta_{\boldsymbol{c}}(c, p)>k-1>0$ and $p \in b_{1}$. Note that $b \cap b_{1}=\emptyset$ since otherwise $\Delta_{\boldsymbol{b}}\left(b_{1}, \operatorname{sh}_{\boldsymbol{b}} \boldsymbol{a}\right) \leq \widetilde{\Delta}_{\boldsymbol{b}}\left(b_{1}, b \cap b_{1}\right)+\Delta_{\boldsymbol{b}}\left(b \cap b_{1}, \operatorname{sh}_{\boldsymbol{b}} \boldsymbol{a}\right) \leq 2$ which is impossible as $\boldsymbol{a}-\boldsymbol{b}-p(k)$ and $k \geq 2$. Thus $b_{1} \subset a$ and $a \cup c=T$. Since $c$ was an arbitrary element of $\mathrm{Sh}_{\boldsymbol{c}} \boldsymbol{b}$, it follows that $\mathrm{Sh}_{\boldsymbol{c}} \boldsymbol{b} \subset \mathrm{Sh}_{\boldsymbol{c}} \boldsymbol{a}$ and $\mathrm{sh}_{\boldsymbol{c}} \boldsymbol{a} \subset \mathrm{sh}_{\boldsymbol{c}} \boldsymbol{b}$. Thus $\Delta_{\boldsymbol{c}}\left(\mathrm{sh}_{\boldsymbol{c}} \boldsymbol{a}, c_{0}\right)>k$.

The above notions allow us to introduce the following relation on the set Ent $T$.

Definition 3.20 (Busemann order). For $\boldsymbol{a}, \boldsymbol{b} \in$ Ent $T$, and $p \in T$ we say that $\boldsymbol{a}$ and $\boldsymbol{b}$ are Busemann ordered with respect to $p$ if

$$
\begin{aligned}
\text { either } \boldsymbol{a} & =\boldsymbol{b}, \\
& \text { or } \boldsymbol{a}-\boldsymbol{b}-p(k) .
\end{aligned}
$$

We will denote this relation by $\boldsymbol{a} \geq_{p, k} \boldsymbol{b}$.

Lemma 3.19 implies that this relation is a partial order on Ent $T$. Using Busemann order we can reformulate the above definitions of conical and parabolic points as follows. 
Lemma 3.21. A point $p \in T$ is A-conical if and only if its Busemann order has no minimal elements. A point $p$ is A-parabolic if and only if its Busemann order has infinitely many minimal elements.

\subsection{Non-refinable tubes}

Lemma 3.22. The set $\Psi_{k}(\boldsymbol{a}, \boldsymbol{b})=\{\boldsymbol{c} \in A: \boldsymbol{a}-\boldsymbol{c}-\boldsymbol{b}(k)\}$ is finite for any $k \geq 1$.

Proof. Suppose that $\boldsymbol{a}-\boldsymbol{c}-\boldsymbol{b}(k)$ and let us prove that $\boldsymbol{c} \#(\boldsymbol{a} \cap \boldsymbol{b})$. If it is not true, then we have $\boldsymbol{c} \bowtie(\boldsymbol{a} \cap \boldsymbol{b})$, i.e. there exists $c \in \operatorname{Small}(\boldsymbol{c}), w \in \operatorname{Small}(\boldsymbol{a} \cap \boldsymbol{b})$ such that $c \cup w=T$. Thus $c \in \mathrm{Sh}_{\boldsymbol{c}} \boldsymbol{a} \cap \mathrm{Sh}_{\boldsymbol{c}} \boldsymbol{b}$ and $\operatorname{sh}_{\boldsymbol{c}} \boldsymbol{a} \subset c, \operatorname{sh}_{\boldsymbol{c}} \boldsymbol{b} \subset c$. Hence $\Delta_{\boldsymbol{c}}\left(\operatorname{sh}_{\boldsymbol{c}} \boldsymbol{a}, \operatorname{sh}_{\boldsymbol{c}} \boldsymbol{b}\right) \leq 1$ which is impossible. It follows that $\boldsymbol{c} \#(\boldsymbol{a} \cap \boldsymbol{b})$. The finiteness of $\Psi_{k}(\boldsymbol{a}, \boldsymbol{b})$ now follows from the discreteness of $A$

Definition 3.23 (refinability). A pair $\{\boldsymbol{a}, \boldsymbol{b}\} \subset A$ is called $(k-$-)refinable if

$$
\Psi_{k}(\boldsymbol{a}, \boldsymbol{b}) \neq \emptyset,
$$

and $(k-)$ non-refinable otherwise.

Proposition 3.25 below guarantees the existence of a finite non-refinable tube between two given entourages in $A$. To prove it we need the following:

Lemma 3.24. For every integer $k \geq 2$, every pair $\{\boldsymbol{a}, \boldsymbol{b}\} \subset A$ is either $(k+1)$-nonrefinable or there exists $\boldsymbol{c} \in \Psi_{k}(\boldsymbol{a}, \boldsymbol{b})$ such that the pair $\{\boldsymbol{a}, \boldsymbol{c}\}$ is $(k+1)$-nonrefinable.

Proof. Suppose this is not true and let a pair $\{\boldsymbol{a}, \boldsymbol{b}\}$ be a counter-example. By Lemma 3.22 the set $\Psi_{k}(\boldsymbol{a}, \boldsymbol{b})$ is finite so we can assume in addition that the number $\left|\Psi_{k}(\boldsymbol{a}, \boldsymbol{b})\right|$ is the minimal one among all such counter-examples. So $\{\boldsymbol{a}, \boldsymbol{b}\}$ is $(k+1)$-refinable and there exists $\boldsymbol{c} \in \Psi_{k+1}(\boldsymbol{a}, \boldsymbol{b})$ such that the pair $(\boldsymbol{a}, \boldsymbol{c})$ is $(k+1)$-refinable too. We now claim that

$$
\Psi_{k+1}(\boldsymbol{a}, \boldsymbol{c}) \subset \Psi_{k+1}(\boldsymbol{a}, \boldsymbol{b})(k>1) .
$$

Let $\boldsymbol{d} \in \Psi_{k+1}(\boldsymbol{a}, \boldsymbol{c})$. By [15, Lemma T2] we have $\boldsymbol{d}-\boldsymbol{c}-\boldsymbol{b}(k)$. Then $\operatorname{sh}_{d} b \subset \operatorname{sh}_{d} c, c f$. [16, Lemma B1]. Therefore $\Delta_{d}\left(\operatorname{sh}_{d} b, \operatorname{sh}_{d} a\right) \geq \Delta_{d}\left(\operatorname{sh}_{d} c, \operatorname{sh}_{d} a\right)$. So $\boldsymbol{d} \in \Psi_{k+1}(\boldsymbol{a}, \boldsymbol{b})$ and (1) follows.

As $\boldsymbol{c} \in \Psi_{k}(\boldsymbol{a}, \boldsymbol{b}) \backslash \Psi_{k}(\boldsymbol{a}, \boldsymbol{c})$ we obtain that $\left|\Psi_{k}(\boldsymbol{a}, \boldsymbol{c})\right|<\left|\Psi_{k}(\boldsymbol{a}, \boldsymbol{b})\right|$. Thus by the minimality of $(\boldsymbol{a}, \boldsymbol{b})$ the pair $(\boldsymbol{a}, \boldsymbol{c})$ cannot be a counter-example. Then $(\boldsymbol{a}, \boldsymbol{d})$ is $(k+1)$-nonrefinable. Since $\boldsymbol{d} \in \Psi_{k+1}(\boldsymbol{a}, \boldsymbol{b}) \subset \Psi_{k}(\boldsymbol{a}, \boldsymbol{b})$ the pair $(\boldsymbol{a}, \boldsymbol{b})$ cannot be a counter-example neither. A contradiction.

For a tube $P=\boldsymbol{a}-\boldsymbol{a}_{1}-\cdots-\boldsymbol{a}_{n}-\boldsymbol{b}$ we denote by $\partial P$ its boundary $\{\boldsymbol{a}, \boldsymbol{b}\}$. 
Proposition 3.25. For every pair $\{\boldsymbol{a}, \boldsymbol{b}\} \subset A$ and integer $k \geq 2$ there exists $a$ finite $k+2$-nonrefinable $k$-tube $P \subset A$ such that $\partial P=\{\boldsymbol{a}, \boldsymbol{b}\}$.

Proof. Suppose this is not true. Let a pair $\{\boldsymbol{a}, \boldsymbol{b}\}$ be a counter-example such that it has the minimal cardinality $\left|\Psi_{k}(\boldsymbol{a}, \boldsymbol{b})\right|$ among all such pairs. Since $\{\boldsymbol{a}, \boldsymbol{b}\}$ is $k+2$-refinable by the above lemma there exists $\boldsymbol{c} \in \Psi_{k+1}(\boldsymbol{a}, \boldsymbol{b})$ such that $\{\boldsymbol{a}, \boldsymbol{c}\}$ is $(k+2)$-nonrefinable. Since the inclusion $\Psi_{k}(\boldsymbol{c}, \boldsymbol{b}) \subset \Psi_{k}(\boldsymbol{a}, \boldsymbol{b})$ is strict there exists a $k+2$-nonrefinable $k$-tube $Q$ with $\partial Q=\{\boldsymbol{c}, \boldsymbol{b}\}$. By the transitivity property [15, Lemma T2] the set $R=\{\boldsymbol{a}\} \cup Q$ is a $k$-tube with the boundary $\{\boldsymbol{a}, \boldsymbol{b}\}$. It is $(k+2)$-nonrefinable by construction. Thus the pair $\{\boldsymbol{a}, \boldsymbol{b}\}$ is not a counterexample. We have a contradiction.

Definition 3.26 (horospherical projection [15]). Let $p \in \mathcal{P}$ be a parabolic point and $T(p)$ be a horosphere at $p$. Define a projection map

$$
\Pi_{p}: A \longrightarrow T(p)
$$

(or $\Pi_{p, k}$ ) called horospherical projection as follows. If $\boldsymbol{a} \notin T_{k}(p)$, then

$$
\Pi_{p}(\boldsymbol{a})=\left\{\boldsymbol{p} \in T_{k}(p): \boldsymbol{a}-\boldsymbol{p}-p(k)\right\} ;
$$

and if $\boldsymbol{a} \in T_{k}(p)$, then

$$
\Pi_{p}(\boldsymbol{a})=\boldsymbol{a} .
$$

Proposition 3.27. Let $\mathcal{P}$ denote the set of parabolic points for the action $G \curvearrowright T$. Then for any constants $k>3$ and $d>0$ the following sets are $G$-finite:

1) $\left\{\{\boldsymbol{c}, \boldsymbol{d}\} \mid \boldsymbol{c} \in \Pi_{p}(g \boldsymbol{a}), \boldsymbol{d} \in \Pi_{p}(g \boldsymbol{b}), p \in \mathcal{P}, g \in G\right\}$ for all $\{\boldsymbol{a}, \boldsymbol{b}\} \subset A$;

2) $\mathcal{A}_{1}=\left\{(\boldsymbol{a}, \boldsymbol{b}) \mid \Psi_{k}(\boldsymbol{a}, \boldsymbol{b})=\emptyset,\{\boldsymbol{a}, \boldsymbol{b}\} \not \subset T_{A, k}(p), p \in \mathcal{P}\right\}$;

3) a) $\left\{\{p, q\} \subset \mathcal{P} \mid N_{d}\left(T_{A, k}(p)\right) \cap N_{d}\left(T_{A, K}(q)\right) \neq \emptyset\right\}$, and

b) $\left\{N_{d}\left(T_{A, k}(p)\right) \cap N_{d}\left(T_{A, k}(q)\right) \mid\{p, q\} \subset \mathcal{P}\right\}$.

Proof. 1) Suppose to the contrary that the set 1) is infinite. Assume first that $\boldsymbol{a} \neq \boldsymbol{b}$. Then there exist an infinite sequence of elements $g_{n} \in G$, distinct entourages $\left\{\boldsymbol{c}_{n}, \boldsymbol{d}_{n}\right\} \subset A$ such that

$$
g_{n} \boldsymbol{a}-\boldsymbol{c}_{n}-p_{n}(k) \text { and } g_{n} \boldsymbol{b}-\boldsymbol{d}_{n}-p_{n}(k),
$$

$\boldsymbol{c}_{n} \in T_{A, k}\left(p_{n}\right), \boldsymbol{d}_{n} \in T_{A, k}\left(p_{n}\right), p_{n} \in \mathcal{P}$. 
Since the set $\mathcal{P}$ is $G$-finite (Lemma 3.17) we can assume that $p_{n}=p$. Since the stabilizer $\operatorname{Stab}_{G} p$ acts cofinitely on $T_{A, k}(p)$ (Lemma 3.17) we can also fix $c_{n}=$ $c \in T_{A, k}(p)$, and assume that $\boldsymbol{d}_{n}=h_{n}(\boldsymbol{d}), \boldsymbol{d} \in T_{A, k}(p), h_{n} \in \operatorname{Stab}_{G} p$. So (2) gives

$$
g_{n} \boldsymbol{a}-\boldsymbol{c}-p(k) \quad \text { and } \quad g_{n} \boldsymbol{b}-\boldsymbol{d}_{n}-p(k),
$$

$c \in T_{A, k}(p), \boldsymbol{d}_{n} \in T_{A, k}(p), p \in \mathcal{P}$.

The following lemma implies that $p$ is a limit point of $\left\{g_{n} \boldsymbol{b}\right\}_{n}$.

Lemma 3.28. If $\boldsymbol{b}_{n}-\boldsymbol{d}_{n}-p(k)(k>1), \boldsymbol{d}_{n} \in T_{A, k}(p)$ and $\lim _{n \rightarrow \infty} \boldsymbol{d}_{n}=p$ then $\lim _{n \rightarrow \infty} \boldsymbol{b}_{n}=p$.

We start with the following claim.

Claim. For every $k>1$ there exists $\boldsymbol{d} \in T_{A, k}(p)$ such that $q-\boldsymbol{d}-p(k)$.

Proof of the claim. Indeed by $m$-separation property (2) there exists $\boldsymbol{a} \in A$ such that $q-\boldsymbol{a}-p(k)$, for $1<k \leq m$. If $\boldsymbol{a} \in T_{A, k}$ we are done. If not let $\boldsymbol{p} \in \Pi_{p}(\boldsymbol{a})$ so $\boldsymbol{a}-\boldsymbol{p}-p(k)$. Let $U_{p}$ be a $\boldsymbol{p}$-small neighborhood of $p$. Let also $b \in \mathrm{Sh}_{\boldsymbol{p}} \boldsymbol{a}$, then $a \cup b=T$ where $b$ is $p$-small and $a=b^{\prime}$ is $\boldsymbol{a}$-small set respectively. We have $\Delta_{\boldsymbol{p}}\left(b, U_{p}\right) \geq \Delta_{\boldsymbol{p}}\left(U_{p}, \operatorname{sh}_{\boldsymbol{p}} \boldsymbol{a}\right)-\widetilde{\Delta}\left(\operatorname{sh}_{\boldsymbol{p}} \boldsymbol{a}, b\right)>k-1$. Therefore $U_{p} \subset a$ and so $U_{p}$ is $\boldsymbol{a}$-small. Then for any $\boldsymbol{a}$-small neighborhood $U_{q}$ of $q$ we have $\Delta_{\boldsymbol{a}}\left(U_{q}, U_{p}\right)>k$. Hence $\Delta_{\boldsymbol{a}}\left(U_{q}, a\right)>k-1$. We have proved that $U_{q} \subset b$ for any $b \in \mathrm{Sh}_{\boldsymbol{p}} \boldsymbol{a}$. Thus $U_{q}$ is $p$-small and $U_{q} \subset \operatorname{sh}_{p} a$. It implies that $\Delta_{p}\left(U_{q}, U_{p}\right) \geq \Delta_{p}\left(\operatorname{sh}_{p} a, U_{p}\right)>k$. The claim follows.

Proof of the Lemma. Suppose by contradiction that there exists an accumulation point $q \in T$ of the set $\left\{\boldsymbol{b}_{n}\right\}_{n}$ distinct from $p$. By the claim there exists $\boldsymbol{p} \in T_{A, k-1}(p)$ such that $q-\boldsymbol{p}-p(k-1)$. Since $\boldsymbol{d}_{n} \rightarrow p$ and $\boldsymbol{b}_{n} \rightarrow q$ by Lemma 3.6 we obtain $\boldsymbol{b}_{n}-\boldsymbol{p}-\boldsymbol{d}_{n}(k-1)\left(n>n_{0}\right)$. So $\operatorname{sh}_{\boldsymbol{d}_{n}} \boldsymbol{p} \supset \operatorname{sh}_{\boldsymbol{d}_{n}} \boldsymbol{b}_{n}$. Since $\boldsymbol{b}_{n}-\boldsymbol{d}_{n}-p(k)$ we obtain $\boldsymbol{p}-\boldsymbol{d}_{n}-p(k-1)$. This is impossible as $\boldsymbol{p} \in T_{A, k-1}(p)$. The lemma is proved.

It follows from $\left(2^{\prime}\right)$ that for any $\left(g_{n} \boldsymbol{a}\right)$-small set $a_{n} \in \mathrm{Sh}_{\boldsymbol{c}}\left(g_{n} \boldsymbol{a}\right)$ and a $\boldsymbol{c}$-small neighborhood $U_{p}$ of $p$ we have $\Delta_{\boldsymbol{d}_{n}}\left(a_{n}, U_{p}\right)>k-1>0(n \in \mathbb{N})$. Thus $U_{p} \subset a_{n}^{\prime}$ and $U_{p}$ is $g_{n} \boldsymbol{a}$-small for all $n \in \mathbb{N}$.

From the other hand by Proposition 3.14 we have that $G \curvearrowright \widetilde{T}$ is a convergence action. Then by [17, Lemma 5.1] for every pair of distinct non-conical points $\{x, y\} \subset \widetilde{T}$ the accumulation points of the orbit $G(x, y)$ belong to the diagonal $\Delta^{2} \tilde{T}$. By Lemma $3.3 \lim _{n \rightarrow \infty} g_{n}(\boldsymbol{b})=p$ so $\lim _{n \rightarrow \infty} g_{n}(\boldsymbol{a})=p$. Hence for the 
above neighborhood $U_{p}$ we also have that $U_{p}^{\prime}$ is $\left(g_{n} \boldsymbol{a}\right)$-small for some $n \in \mathbb{N}$. This is impossible by our convention (1) of Section 3.1. Part 1) is proved.

2) Suppose that $\left\{\left(\boldsymbol{a}_{i}, \boldsymbol{b}_{i}\right) \in A \times A \mid i \in I\right\}$ is an infinite set such that for every $i \in I$ there is no $\boldsymbol{c}_{i} \in A$ such that $\boldsymbol{a}_{i}-\boldsymbol{c}_{i}-\boldsymbol{b}_{i}(k)$. The set $A$ is $G$-finite so we can fix $\boldsymbol{a}=\boldsymbol{a}_{i}$ and assume that $\boldsymbol{b}_{i}=g_{i}(\boldsymbol{b}): g_{i} \in G$. Since the space $\tilde{T}$ is compact, the set $\left\{\boldsymbol{b}_{i}\right\}_{i \in I}$ admits an accumulation point $p$ which is a limit point for the geometrically finite action $G \curvearrowright \widetilde{T}$. By Lemma $3.17 p$ is either $k$-conical or $k$-parabolic point for some (any) $k>1$. Consider these two cases separately.

Let first, $p$ be a $k$-conical point. Then there exists $c \in A$ such that $\boldsymbol{a}-\boldsymbol{c}-p(k)$. By Lemma 3.6 we have $\boldsymbol{a}-\boldsymbol{c}-\boldsymbol{b}_{i}(k)(i \in I)$ contradicting the $k$-non-refinability of the pair $\left\{\boldsymbol{a}, \boldsymbol{b}_{i}\right\}$.

Let us now suppose that $p$ is $k$-parabolic. We will now show that for almost all $i \in I$ the entourages $\boldsymbol{a}$ and $\boldsymbol{b}_{i}$ belong to the same horosphere $T_{A, k}(p)$. We claim first that $a \in T_{A, k}(p)$. Indeed if not, then there exists $c \in A$ such that $\boldsymbol{a}-\boldsymbol{c}-p(k)$ contradicting by the same argument the $k$-non-refinability of the pair $\left\{\boldsymbol{a}, \boldsymbol{b}_{i}\right\}(i \in I)$. So $\boldsymbol{a} \in T_{A, k}(p)$.

Suppose by contradiction that there exist $\boldsymbol{b}_{i} \notin T_{A, k}(p)$ for infinitely many $i \in I$. Then there exist $c_{i} \in T_{A, k}(p)$ such that

$$
\boldsymbol{b}_{i}-\boldsymbol{c}_{i}-p(k)
$$

We first note that in $(*)$ we cannot have the same entourage $c_{0}$ for infinitely many different $\boldsymbol{b}_{i}$. Indeed if not, then from $(*)$ we have $\Delta_{\boldsymbol{c}_{0}}\left(\mathrm{sh}_{\boldsymbol{c}_{0}} \boldsymbol{b}_{i}, c_{0}\right)>k$ $(i \in I)$ for a $c_{0}$-small set $c_{0}$ containing $p$. Since $p$ is an accumulation point for the set $\left\{\boldsymbol{b}_{i}\right\}_{i \in I}$ then $c_{0}^{\prime}$ is $\boldsymbol{b}_{i}$-small for infinitely many $i \in I$. Thus $c_{0} \supset \operatorname{sh}_{\boldsymbol{c}_{0}} \boldsymbol{b}_{i}$, and $\Delta_{\boldsymbol{c}_{0}}\left(c_{0}, \mathrm{sh}_{\boldsymbol{c}_{0}} \boldsymbol{b}_{i}\right) \leq 1$ which is impossible.

So we can assume that $c_{i}$ are all distinct. By Lemma 3.17 the quotient $T_{A, k}(p) / \operatorname{Stab}_{\mathrm{G}} \mathrm{p}$ is finite, so there exists $h_{i} \in \operatorname{Stab}_{\mathrm{G}} \mathrm{p}$ such that $h_{i}\left(c_{i}\right)=c \in$ $T_{A, k}(p)$. Hence $h_{i}\left(\boldsymbol{b}_{i}\right)-\boldsymbol{c}-p(k)$ for every $i \in I_{1}$ where $I_{1}$ is an infinite subset of $I$. Since $\boldsymbol{a} \in T_{A, k}(p)$ by Lemma $3.18 p$ is an accumulation point for the set $\left\{h_{i}(\boldsymbol{a})\right\}_{i \in I_{1}}$. Then by Lemma 3.6 we obtain $h_{i}\left(\boldsymbol{b}_{i}\right)-\boldsymbol{c}-h_{i}(\boldsymbol{a})(k)$ and so $\boldsymbol{b}_{i}-h_{i}^{-1} \boldsymbol{c}_{i}-\boldsymbol{a}(k)$ which is impossible.

So $\boldsymbol{b}_{i} \in T_{A, k}(p)$ for almost all $i \in I$. This shows that the set $A_{1}$ is $G$-finite. Part 2) is proved.

3) a) We omit the index $k$ below. Suppose that the first set is infinite. Then there exists an infinite set of $G$-non-equivalent pairs of parabolic points $\left(p_{i}, q_{i}\right) \in \mathcal{P}^{2}$ for which $N_{d}\left(T\left(p_{i}\right)\right) \cap N_{d}\left(T\left(q_{i}\right)\right) \neq \varnothing(i \in I)$. Since the action of $G$ on $\Theta^{2} T$ is cocompact there exist $g_{i} \in G$ such that the pair $\left(g_{i}\left(p_{i}\right), g_{i}\left(q_{i}\right)\right)$ belong to 
a compact subset of $\Theta^{2} T$. So without lost of generality we may assume that the sets $\left\{p_{i}\right\}_{i \in I}$ and $\left\{q_{i}\right\}_{i \in I}$ admits two distinct accumulation points $p$ and $q$. It follows from [15, Lemma P3] that there cannot exist an entourage belonging to the intersection of infinitely many distinct horospheres (for a more general system of horospheres this is also true, see [18, Corollary of 4.4.2]). So there is an infinite sequence of distinct entourages $\boldsymbol{b}_{i} \in N_{d}\left(T\left(p_{i}\right)\right) \cap N_{d}\left(T\left(q_{i}\right)\right)(i \in I)$. The set $\left\{\boldsymbol{b}_{i}\right\}_{i \in I}$ admits an accumulation point $x \in T$. Let $\left(\boldsymbol{c}_{i}\right)_{i} \subset T\left(p_{i}\right)$ and $\left(\boldsymbol{d}_{i}\right)_{i} \subset T\left(q_{i}\right)$ be two subsets for which $d_{A}\left(\boldsymbol{b}_{i}, \boldsymbol{c}_{i}\right)$ and $d_{A}\left(\boldsymbol{b}_{i}, \boldsymbol{d}_{i}\right)$ are bounded by the constant $d$. Thus $d_{A}\left(\boldsymbol{c}_{i}, \boldsymbol{d}_{i}\right) \leq 2 d$ and by Lemma 3.15 we have $p=q=x$. A contradiction.

b) If now the second set is not $G$-finite then for a fixed parabolic point $p \in \mathcal{P}$ by the part a) we obtain $q \in \mathcal{P}$ such that the set $N_{d}(T(p)) \cap N_{d}(T(q))$ is infinite. Then by 3.15 we must have $p=q$. The proposition is proved.

Corollary 3.29. Suppose that $G$ is a finitely generated group acting 3-discontinuously and 2-cocompactly on a compactum $T$. Then there exists a constant $C>0$ such that the $d_{A}$-diameter of each of the sets 1), 2), and 3b) of Proposition 3.27 is bounded by $C$.

Proof. Since $G$ is finitely generated by Lemma 3.11 the graph $\mathcal{G}$ is connected. So $d$ is a real distance. The Corollary follows from the above proposition.

From Proposition 3.27,2) we immediately have

Corollary 3.30. Let $G \curvearrowright T$ be a 3-discontinuous and 2-cocompact action satisfying the above conditions. Then if for a fixed $\boldsymbol{a} \in A$ and infinitely many $\boldsymbol{b}_{n} \in A$ the pairs $\left(\boldsymbol{a}, \boldsymbol{b}_{n}\right)$ are all non-refinable then for all but finitely many $n$ one has $\left(a, b_{n}\right) \subset T(p)$.

We will now obtain few more finiteness properties characterizing the horospherical projection $\Pi_{p}: A \rightarrow T_{A, k}(p)(p \in \mathcal{P})$. The following definition is motivated by Lemma 3.6.

Definition 3.31. For a fixed $k>3$ a visibility neighborhood of the point $\boldsymbol{p} \in$ $\Pi_{p}(\boldsymbol{a}) \subset T_{A, k}(p)$ from the point $\boldsymbol{a} \in A$ is the set

$$
\mathcal{N}(\boldsymbol{a}, \boldsymbol{p}, p)=\left\{\boldsymbol{x} \in T_{A, k}(p) \mid \boldsymbol{a}-\boldsymbol{p}-p(k) \wedge \neg \boldsymbol{a}-\boldsymbol{p}-\boldsymbol{x}(k-1)\right\},
$$

where $\neg$ denotes the opposite logical statement. 
The following proposition establishes the $G$-finiteness properties of two more sets (by continuing the notations of 3.27):

Proposition 3.32. For every $k>1$ the following sets are $G$-finite:

1) $\mathcal{A}_{2}=\left\{(\boldsymbol{x}, \boldsymbol{p}) \in T_{k}^{2}(p) \mid \boldsymbol{x} \in \mathcal{N}(\boldsymbol{a}, \boldsymbol{p}, p), \boldsymbol{a} \in A, p \in \mathcal{P}\right\}$;

2) $\mathcal{A}_{3}=\left\{\Pi_{p}\left(T_{k}(q)\right) \mid\{p, q\} \subset \mathcal{P}\right\}$.

Proof. 1) Suppose by contradiction that it is not true and $\mathcal{A}_{2}$ is not $G$-finite for some $k>1$. Since $A$ is one $G$-orbit up to taking an infinite subset of $\mathcal{A}_{2}$ we can fix the entourage $\boldsymbol{p}$. By [15, Lemma P3] $\boldsymbol{p}$ can belong to at most finitely many different horospheres. So up to a passing to a new infinite subset we can fix the parabolic point $p \in \mathcal{P}$.

If first the set of entourages $\left\{\boldsymbol{a} \mid\left(\boldsymbol{x}, \Pi_{p}(\boldsymbol{a})\right) \in \mathcal{A}_{2}\right\}$ is finite, up to choosing a new infinite subset of $\mathcal{A}_{2}$ we have $\boldsymbol{a}-\boldsymbol{p}-p(k)$ and $\neg \boldsymbol{a}-\boldsymbol{p}-\boldsymbol{x}(k-1)$ for a fixed $\boldsymbol{a}$. Then the set of the first coordinates $\left\{\boldsymbol{x} \mid(\boldsymbol{x}, \cdot) \in \mathcal{A}_{2}\right\} \subset T(p)$ is infinite and by Lemma 3.18 its accumulation point is $p$. Then by Lemma 3.6 there exists $\boldsymbol{x}$ in this set such that $\boldsymbol{a}-\boldsymbol{p}-\boldsymbol{x}(k)$. A contradiction.

If now the set $\left\{\boldsymbol{a} \mid\left(\boldsymbol{x}, \Pi_{p}(\boldsymbol{a})\right) \in \mathcal{A}_{2}\right\}$ is infinite let $q \in T$ be its accumulation point. Taking a $\boldsymbol{p}$-small neighborhood $U_{q}$ of $q$ we obtain that $U_{q}^{\prime}$ is $\boldsymbol{a}$-small for every $\boldsymbol{a} \in U_{q}$. Thus $U_{q} \supset \operatorname{sh}_{\boldsymbol{p}} \boldsymbol{a}$. Since $\boldsymbol{a}-\boldsymbol{p}-p(k)$, so $\Delta_{\boldsymbol{p}}\left(U_{q}, U_{p}\right)>k-1$ for a $\boldsymbol{p}$-small neighborhood $U_{p}$ of $p$. It yields $q-\boldsymbol{p}-p(k-1)$. There are infinitely many $\boldsymbol{x} \in T(p)$ corresponding to the points $\boldsymbol{a} \in U_{q}$. Since $p$ is the unique accumulation point of $T(p)$ we must have $\boldsymbol{x} \in U_{p}$ for most such $\boldsymbol{x}$. Hence $\Delta\left(\operatorname{sh}_{\boldsymbol{p}} \boldsymbol{a}, \operatorname{sh}_{\boldsymbol{p}} \boldsymbol{x}\right) \geq$ $\Delta_{\boldsymbol{p}}\left(U_{p}, U_{q}\right)>k-1$. Therefore $\boldsymbol{a}-\boldsymbol{p}-\boldsymbol{x}(k-1)$. Again a contradiction.

2) Suppose not. Since the set of parabolic points $\mathcal{P}$ is $G$-finite we can fix the point $p \in \mathcal{P}$. Using the action of $\operatorname{Stab}_{G} p$ on $T_{k}(p)$ we can also assume that there is a fixed entourage $c \in T(p)$ such that for every $q \in \mathcal{P}: c \in \Pi_{p}(T(q))$. So there exists an infinite set $\left\{\boldsymbol{d}_{i} \in \Pi_{p}\left(T\left(q_{i}\right)\right) \mid i \in I, q_{i} \in \mathcal{P}\right\}$ such that for all $i \in I$ we have

$$
\boldsymbol{b}_{i}-\boldsymbol{d}_{i}-p(k), \quad \boldsymbol{a}_{i}-\boldsymbol{c}-p(k), \quad\left\{\boldsymbol{a}_{i}, \boldsymbol{b}_{i}\right\} \subset T\left(q_{i}\right) .
$$

Since $p$ is the unique accumulation point of $T(p)$, up to passing to an infinite subsequence of $I$, we may assume that $\lim _{i \rightarrow \infty} \boldsymbol{d}_{i}=p$. Then by Lemma 3.3 we have $\lim _{i \rightarrow \infty} \boldsymbol{b}_{i}=p$. Let $q \in T$ be an accumulation point of the set $\left\{q_{i}\right\}_{i \in I}$. 
We claim that $q=p$. Indeed if not then there exists an entourage $\boldsymbol{a} \in A$ such that $q-\boldsymbol{a}-p(k)$. Hence for infinitely many $i \in I$ we have $q-\boldsymbol{a}-\boldsymbol{b}_{i}(k)$ (Lemma 3.6). Then $\Delta_{\boldsymbol{a}}\left(U_{q}, \operatorname{sh}_{\boldsymbol{a}} \boldsymbol{b}_{i}\right)>k$ for every $\boldsymbol{a}$-small neighborhood $U_{q}$ of $q$. So for some $i \in I$ we have $q_{i} \in U_{q}$ and hence $q_{i}-\boldsymbol{a}-\boldsymbol{b}_{i}(k)$. The latter one is impossible since $\boldsymbol{b}_{i} \in T_{k}\left(q_{i}\right)$. If the set $\left\{\boldsymbol{a}_{i}\right\}$ has an accumulation point $r$ different from $p$ then there exists $\boldsymbol{a} \in A$ such that $r-\boldsymbol{a}-p(k)$. So as above we have $q_{i}-\boldsymbol{a}-\boldsymbol{a}_{i}(k)$ which is impossible by the same reason. So $\lim _{i \rightarrow \infty} \boldsymbol{a}_{i}=p$. Then for every $\boldsymbol{c}$-small neighborhood $U_{p}$ of $p$ we have that $U_{p}$ is also $\boldsymbol{a}_{i}$-small and $U_{p}^{\prime}$ is $\boldsymbol{a}_{i}$-small for infinitely many $i$. This is impossible. The proposition is proved.

The following Corollary gives a uniform bound on the cardinality of the intersection of the stabilizers of parabolic points for a geometrically finite action.

Corollary 3.33. Let $G$ be a group admitting a 3-discontinuous and 2-cocompact action on a compactum $T$. Then there is a constant $C$ such that for every pair of distinct parabolic points $p_{i}$ and $p_{j}$ for the action $G \curvearrowright T$ one has

$$
\left|\operatorname{Stab}_{G} p_{i} \cap \operatorname{Stab}_{G} p_{j}\right| \leq C .
$$

Proof. Denote $H_{i}=\operatorname{Stab}_{G} p_{i}$. Suppose the statement is not true. By Lemma 3.17 the set of parabolic points for the action $G \curvearrowright T$ is $G$-finite. So up to conjugation we can suppose that there exists a sequence of the stabilizers of parabolic points $H_{0}, H_{n}(n \in \mathbb{N})$ such that $\left|H_{0} \cap H_{n}\right| \rightarrow \infty$. Let $T_{n}$ be a horosphere at $p_{n}(n \in$ $\mathbb{N} \cup\{0\})$. Then the projection $\Pi_{p_{0}}\left(T_{n}\right)$ of $T_{n}$ on $T_{0}$ is invariant under $H_{0} \cap H_{n}$. Since the action $H_{0} \curvearrowright T_{0}$ is discontinuous we have $\left|\Pi_{p_{0}}\left(T_{n}\right)\right| \rightarrow \infty$ which is impossible by Proposition 3.32.2).

Remark. The above corollary is also true if $G$ is a countable group acting 3-discontinuously on a compactum $T$ such that every point $T$ is either conical or bounded parabolic. Indeed in this case by [15, Main Theorem (c)] the space $T$ is metrisable. So by [29] the action $G \curvearrowright T$ is 2-cocompact and the above Corollary holds.

3.4. Proof of Theorem A. The aim of this subsection is the following.

Theorem A. Let $G$ be a relatively hyperbolic group with respect to a collection of parabolic subgroups $\left\{P_{1}, \ldots, P_{n}\right\}$. Then $G$ is the fundamental group of the following finite "star graph" 


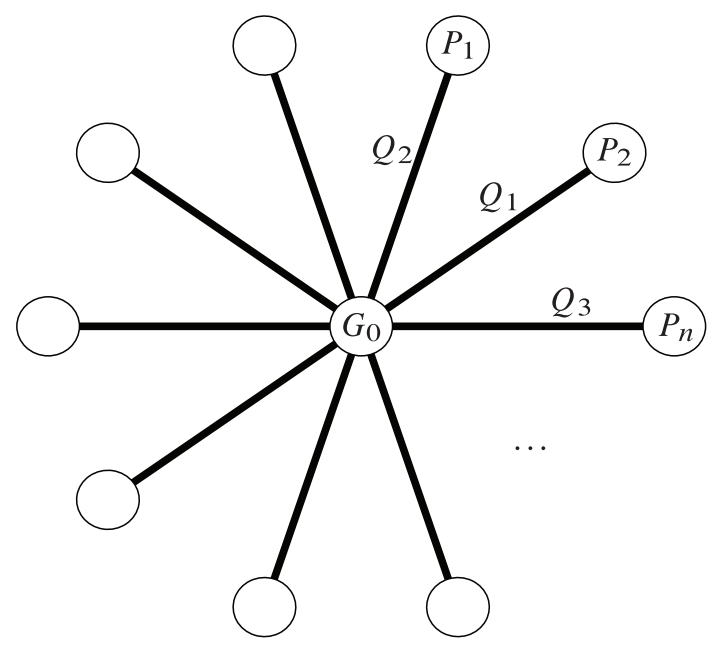

whose central vertex group $G_{0}$ is finitely generated relatively hyperbolic with respect to those edge groups $Q_{i}=P_{i} \cap G_{0}$ which are infinite, all other vertex groups of the graph are $P_{i}(i=1, \ldots, n)$.

Moreover for every finite set $K \subset G$ the subgroup $G_{0}$ can be chosen to contain $K$.

Proof. Recall that $A=G\left(a_{0}\right)\left(a_{0} \in\right.$ Ent $\left.T\right)$ is a discrete orbit of entourages forming the vertex set of the graph $\mathcal{G}$ satisfying our Convention 3.12. Without lost of generality we can assume that the group $G$ is not finitely generated and $\boldsymbol{a}_{0} \in K$. So the graph $\mathcal{G}$ is not connected (see Lemma 3.11). The distance $d_{A}(\boldsymbol{x}, \boldsymbol{y})$ is a pseudo-distance being infinity if and only if $\boldsymbol{x}$ and $\boldsymbol{y}$ belong to different connected components of $\mathcal{G}$. By Lemmas 3.17 and 3.18 the set $\mathcal{P}$ of parabolic points for the action $G \curvearrowright T$ is $G$-finite; and for every $p \in \mathcal{P}$ the stabilizer $H_{p}=\operatorname{Stab}_{\mathrm{G}} \mathrm{p}$ acts cofinitely on its horosphere $T(p)$.

Let $\mathcal{A}_{i}(i=1,2,3) \subset A^{2}$ be the $G$-finite sets introduced in Propositions 3.27.2) and 3.32.

We now construct a new graph $\widetilde{\mathcal{G}}$ whose set of vertices is $A$ and the set of edges is given by the pairs of entourages belonging to the following sets:

a) the finite set $K^{2}$ and the set of all its horospherical projections

$$
\left\{\Pi_{p}\left(K^{2}\right) \mid p \in \mathcal{P}\right\}
$$

b) the set $\mathcal{A}_{1}$ and the set of all its horospherical projections $\left\{\Pi_{p}\left(\mathcal{A}_{1}\right) \mid p \in \mathcal{P}\right\}$;

c) the set $\mathcal{A}_{2}$;

d) the set $\mathcal{A}_{3}$. 
All these sets are $G$-finite. Indeed the set $\mathcal{A}_{1}$ is $G$-finite by Proposition 3.27.2). So by Proposition 3.27.1) the set $\left\{\Pi_{p}\left(\mathcal{A}_{1}\right) \mid p \in \mathcal{P}\right\}$ consisting of the projections of finitely many $G$-orbits of pairs is $G$-finite too. The sets $\mathcal{A}_{2}$ and $\mathcal{A}_{3}$ are $G$-finite by Proposition 3.32 .

Lemma 3.34. There exists a finitely generated subgroup $G_{0}$ of $G$ containing any finite subset $K \subset G$ and which is relatively hyperbolic with respect to $Q_{i}=$ $P_{i} \cap G_{0}(i=1, \ldots, n)$.

Proof. Let $\mathcal{G}_{0}$ be the connected component of $\widetilde{\mathcal{G}}$ containing $K$. Set $G_{0}=\operatorname{Stab}_{G} \mathcal{G}_{0}$ and $A_{0}=\mathcal{G}_{0}^{0}$. By Lemma 3.11 the group $G_{0}$ is finitely generated. We are left to prove that $G_{0}$ is relatively hyperbolic with respect to the subgroups $\left\{Q_{i}\right\}_{i=1}^{k}$.

Let $T_{0}$ be a subset of $T$ which is the limit set of $G_{0}$. We will first show that the action $G_{0} \curvearrowright T_{0}$ is 2-cocompact. By [15, Proposition E] the 2-cocompactness is equivalent to the $k$-separation property: there exists $\boldsymbol{b} \in A_{0}$ such that

$$
p-\boldsymbol{b}-q(k), \quad \text { for all } p, q \in T_{0}, p \neq q,
$$

for some $k>0$. Since the action of $G$ on $T$ is 2-cocompact, property (1) is true for some $\boldsymbol{b} \in A$. If $\boldsymbol{b} \in A_{0}$ we are done, so suppose that $\boldsymbol{b} \notin A_{0}$. Let $U_{p}$ and $U_{q}$ be $\boldsymbol{b}$-small neighborhoods of the points $p$ and $q$ such that $\Delta_{\boldsymbol{b}}\left(U_{p}, U_{k}\right)>k$. Since $p$ and $q$ are accumulation points of $A_{0}$ there exist entourages $\boldsymbol{a}, \boldsymbol{c} \in A_{0}$ such that $U_{p}^{\prime}$ is $\boldsymbol{a}$-small and $U_{q}^{\prime}$ is $\boldsymbol{c}$-small. So $U_{p} \supset \operatorname{sh}_{\boldsymbol{b}} \boldsymbol{a}$ and $U_{q} \supset \operatorname{sh}_{\boldsymbol{b}} \boldsymbol{c}$. Hence

$$
\boldsymbol{a}-\boldsymbol{b}-\boldsymbol{c}(k)
$$

By Proposition 3.25 up to refining the pair $\{\boldsymbol{a}, \boldsymbol{b}\}$ we can suppose that the pair $\{\boldsymbol{a}, \boldsymbol{b}\}$ is $k+2$-nonrefinable. Since $\boldsymbol{b} \notin A_{0}$, by operation b) above the pair $\{\boldsymbol{a}, \boldsymbol{b}\}$ must belong to an horosphere $T_{k+2}(r)(r \in \mathcal{P})$. As $\{\boldsymbol{a}, \boldsymbol{c}\} \subset A_{0}$ and $\mathcal{G}_{0}$ is connected there exists a path $\gamma=\gamma(\boldsymbol{a}, \boldsymbol{c}) \subset \mathcal{G}_{0}$. Let $\boldsymbol{e}=\Pi_{r}(\boldsymbol{c})$. Note that for every edge $l \in \mathcal{G}_{0}^{1}$ we have $\Pi_{r}(l) \in \mathcal{G}_{0}^{1}$. Indeed if $l$ joins two vertices of $A_{0}$ then by the operations a), b) and d) all their horospherical projections are joined by edges too. So $\Pi_{r}\left(\mathcal{G}_{0}\right) \subset \mathcal{G}_{0}$. Since $\{\boldsymbol{a}, \boldsymbol{e}\} \subset T(r) \cap \Pi_{r}(\gamma)$ we have $\boldsymbol{e} \in A_{0}$.

Operation c) then implies that $\boldsymbol{b} \notin \mathcal{N}(\boldsymbol{c}, \boldsymbol{e}, r)$. By Definition 3.31 we have

$$
\boldsymbol{b}-\boldsymbol{e}-\boldsymbol{c}(k+1)
$$

So $\operatorname{sh}_{\boldsymbol{b}} \boldsymbol{c} \subset \operatorname{sh}_{\boldsymbol{b}} \boldsymbol{e}$ and (2) yields $\Delta_{\boldsymbol{b}}\left(\operatorname{sh}_{\boldsymbol{b}} \boldsymbol{a}, \operatorname{sh}_{\boldsymbol{b}} \boldsymbol{e}\right)>k-1$ and $\boldsymbol{a}-\boldsymbol{b}-\boldsymbol{e}(k-1)$. Thus $\operatorname{sh}_{\boldsymbol{e}} \boldsymbol{a} \subset \operatorname{sh}_{\boldsymbol{e}} \boldsymbol{b}$ and by (3) we have $\boldsymbol{a}-\boldsymbol{e}-\boldsymbol{c}(k-1)$ with $\boldsymbol{e} \in A_{0}$. We have proved that the action $G_{0} \curvearrowright T_{0}$ is $(k-1)$-separating and so is 2-cocompact; cf. [15, Proposition E]. 
By [15, Main Theorem] every point of $T_{0}$ is either conical or parabolic for the action of $G_{0}$ on $T_{0}$. Let $p \in T_{0}$ be a parabolic point for this action. We need the following.

Claim. The point $p$ is also parabolic for the action of $G$ on $T$.

Proof of the claim. Suppose that the claim is false. Let $T(p) \subset A_{0}$ be a horosphere for the action $G_{0} \curvearrowright \mathcal{G}_{0}$. Let us choose $\boldsymbol{b} \in T_{k-3}(p) \subset T(p)(k>3)$ where $T_{s}(\cdot)$ denotes the "sub-horosphere" of $T(\cdot)$ of order $s$ (see Definition 3.16).

Suppose first that $\boldsymbol{b}$ does not belong to any horosphere $\widetilde{T}_{k}(q) \subset A$ for the action $G \curvearrowright \widetilde{\mathcal{G}}$. Since $p$ is conical for the action $G \curvearrowright T$ there exists $c \in A$ such that $\boldsymbol{b}-\boldsymbol{c}-p(k-1)$. Note that $\boldsymbol{c} \notin A_{0}$ as otherwise $\boldsymbol{b} \notin T_{k-1}(p)$ which is impossible as $T_{k-3}(p) \subset T_{k-1}(p)$. By the sublemma below we can also suppose up to refining the couple $(\boldsymbol{b}, \boldsymbol{c})$ that it is not $k$-refinable $(k>3)$. Since $\boldsymbol{b}$ and $\boldsymbol{c}$ do not belong to one horosphere in $\widetilde{\mathcal{G}}$, by operation b) above, $\boldsymbol{c}$ and $\boldsymbol{b}$ are joined by an edge in $\widetilde{\mathcal{G}}$. So $c \in A_{0}$ and we have a contradiction in this case.

We affirm now that there exists $h \in \operatorname{Stab}_{G_{0}} p$ such that $h(\boldsymbol{b})$ does not belong to any horosphere $\widetilde{T}_{k}(q)$ where $q \in \mathcal{P}$. Suppose not, then $\boldsymbol{b} \in T_{k-3}(p) \cap \widetilde{T}_{k}(q)$ for some $q \in \mathcal{P}$. Again since $p$ is conical for the action on $T$ there exists $c \in A \backslash A_{0}$ such that $\boldsymbol{b}-\boldsymbol{c}-p(k-1)$. By the argument above we can assume that $c \in \widetilde{T}_{k}(q)$ too. Up to choosing $h \in \mathrm{Stab}_{G_{0}} p$ so that $\boldsymbol{b}_{1}=h(\boldsymbol{b}) \in T_{k-3}(p)$ is sufficiently close to $p$ we can also assume that $\boldsymbol{b}-\boldsymbol{c}-\boldsymbol{b}_{1}(k-1)$ (Lemma 3.6). As the distance $d_{A_{0}}\left(\boldsymbol{b}, \boldsymbol{b}_{1}\right)$ is large, by Proposition 3.27.3b) we have that $\boldsymbol{b}_{1} \notin \widetilde{T}_{k}(q)$. Then there exists $q_{1} \in \mathcal{P} \backslash\{p, q\}$ such that $\boldsymbol{b}_{1} \in \widetilde{T}_{k}\left(q_{1}\right)$. By the argument above giving formula (3) it follows that there exists $\boldsymbol{e} \in \Pi_{q}\left(\boldsymbol{b}_{1}\right) \cap A_{0}$ such that $\boldsymbol{b}_{1}-\boldsymbol{e}-\boldsymbol{c}(k-1)$ and so $\boldsymbol{b}-\boldsymbol{e}-\boldsymbol{b}_{1}(k-2)$. Continuing in this way we obtain an infinite sequence $\boldsymbol{b}_{n}=h_{n}(\boldsymbol{b}) \in T_{k-3}(p) \cap \widetilde{T}_{k}\left(q_{n}\right)$ where $h_{n} \in \operatorname{Stab}_{G_{0}} p$ and $q_{n}=h_{n}(q)$ are all different parabolic points. By Proposition 3.27.1) it follows that the subset $B=$ $\bigcup_{n \in \mathbb{N}} \Pi_{q}\left(h_{n}(\boldsymbol{b})\right)$ of $\widetilde{T}_{k}(q) \cap A_{0}$ is finite. So up to choosing a new subsequence for a fixed $\boldsymbol{e} \in B$ we have $\boldsymbol{b}-\boldsymbol{e}-\boldsymbol{b}_{n}(k-2)(n \in \mathbb{N})$. Since $p$ is the accumulation point of $\left\{b_{n}\right\}_{n \in \mathbb{N}}$, for any $\boldsymbol{e}$-small neighborhood $U_{p}$ of $p$ its complement $U_{p}^{\prime}$ is $\boldsymbol{b}_{n}$-small for infinitely many $n$. Thus $U_{p} \supset \operatorname{sh}_{\boldsymbol{e}} \boldsymbol{b}_{n}$ and so $\Delta_{\boldsymbol{e}}\left(\operatorname{sh}_{\boldsymbol{e}} \boldsymbol{b}, U_{p}\right)>k-3$ implying $\boldsymbol{b}-\boldsymbol{e}-p(k-3)$. Therefore $\boldsymbol{b} \notin T_{k-3}(p)$ which is a contradiction proving the claim.

We have $\operatorname{Stab}_{G_{0}} p=\operatorname{Stab}_{G} p \cap G_{0}$. Lemma 3.34 is proved modulo the following lemma. 
Sublemma 3.35. If $\boldsymbol{b}-\boldsymbol{c}-p(k-1)$ and $\boldsymbol{b}-\boldsymbol{c}_{1}-\boldsymbol{c}(k)$ then $\boldsymbol{b}-\boldsymbol{c}_{1}-p(k-1)(k>3)$.

Proof. Let us first show that $c_{1}-c-p(k-2)$. Indeed the second assumption implies that $\operatorname{sh}_{c} c_{1} \supset \operatorname{sh}_{c} b$. So for a $c$-small neighborhood $U_{p}$ of $p$ using the first assumption for any $c \in \mathrm{Sh}_{c} c_{1}$ we have

$$
\Delta_{c}\left(c, U_{p}\right)>\Delta_{c}\left(\operatorname{sh}_{c} b, U_{p}\right)-\widetilde{\Delta}_{c}\left(\operatorname{sh}_{c} b, c\right)>k-2 .
$$

So $U_{p} \subset c^{\prime} \in \operatorname{Sh}_{c_{1}} c$ and $\widetilde{\Delta}_{c_{1}}\left(\operatorname{sh}_{c_{1}} c, U_{p}\right) \leq 1$. Hence

$$
\Delta_{\boldsymbol{c}_{1}}\left(\operatorname{sh}_{\boldsymbol{c}_{1}} \boldsymbol{b}, U_{p}\right)>\Delta_{\boldsymbol{c}_{1}}\left(\mathrm{sh}_{\boldsymbol{c}_{1}} \boldsymbol{b}, \mathrm{sh}_{\boldsymbol{c}_{1}} \boldsymbol{c}\right)-\Delta_{\boldsymbol{c}_{1}}\left(\mathrm{sh}_{\boldsymbol{c}_{1}} \boldsymbol{c}, U_{p}\right)>k-1 .
$$

The lemma and the proposition are proved.

The following lemma finishes the proof of the theorem.

Lemma 3.36. The action $G \curvearrowright \widetilde{\mathcal{G}}$ induces an action on a bipartite simplicial tree $\mathcal{T}$ such that the graph $X=\mathcal{T} / G$ satisfies Theorem A.

Proof. Using the graph $\widetilde{\mathcal{G}}$ we construct the tree $\mathcal{T}$ to have vertices belonging to two subsets $\mathcal{C}$ and $\mathcal{H}$. The elements of $\mathcal{C}$ are components of $\widetilde{\mathcal{G}}$ and the elements of $\mathcal{H}$ are the horospheres of $A=\widetilde{\mathcal{G}}^{0}$. We call them non-horospherical and horospherical respectively. Two vertices $C$ and $H$ of $\mathcal{T}$ are joined by an edge if and only if $C \in \mathcal{C}$, $H \in \mathcal{H}$, and $C \cap H \neq \emptyset$.

Let us first show that $\mathcal{T}$ is connected. Indeed by construction every horospherical vertex is joined with a non-horospherical one. So it is enough to prove that every two non-horospherical vertices can be joined by a path. Let $C_{i}(i=1,2)$ be the corresponding connected components of $\widetilde{\mathcal{G}}$ and let us fix two entourages $\boldsymbol{a} \in C_{1}^{0}$ and $\boldsymbol{b} \in C_{2}^{0}$. By Proposition 3.25 there exists a non-refinable tube between them: $P=\boldsymbol{a}-\boldsymbol{b}_{1}-\cdots-\boldsymbol{b}_{n}-\boldsymbol{b} \subset A$. By operation b) above every non-refinable pair $\left(\boldsymbol{b}_{i}, \boldsymbol{b}_{i+1}\right)$ either belongs to an horosphere $T(p)$ or corresponds to an edge in the graph $\widetilde{\mathcal{G}}$. In the latter case it stays in the same component of $\widetilde{\mathcal{G}}$. In the former case the horosphere $T(p)$ corresponds to a single vertex of the graph $\mathcal{T}$. So the tube $P$ produces a path in $\mathcal{T}$ between the corresponding vertices. Thus $\mathcal{T}$ is connected.

Let us now show that $\mathcal{T}$ is a tree. Suppose not and it contains a simple loop $\alpha$. Since the vertices of two types alternate on $\alpha$ we can fix a horospherical vertex $H$ corresponding to the horosphere $T(p)$ and having two non-horospherical neighboring vertices $C_{1}$ and $C_{2}$. Let $\alpha_{1}$ be a subpath of $\alpha$ containing the vertices $H, C_{1}, C_{2}$, and $\alpha_{2}$ be the closure of $\alpha \backslash \alpha_{1}$. The path $\alpha_{2}$ corresponds to an alternating sequence of components of $\widetilde{\mathcal{G}}$ and horospheres. So we can choose 


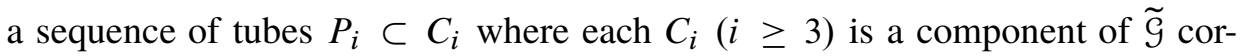
responding to a non-horospherical vertex of $\alpha_{2}$. The tube $P_{i}$ connects two entourages from $C_{i}$ each belonging to horospheres $T\left(q_{i}\right)$ and $T\left(q_{i}^{\prime}\right)$ intersecting $C_{i}$. Note that these horospheres differ from the initial horosphere $T(p)$ as $\alpha$ is a simple loop. By operations b) and d) above it follows that that there exists the path $\cup_{i} \Pi_{p}\left(P_{i} \cup T\left(q_{i}\right) \cup T\left(q_{i}^{\prime}\right)\right)$ on $T(p) \cap \widetilde{\mathcal{G}}$. It implies that the vertices $C_{1}$ and $C_{2}$ correspond to the same connected component of $\widetilde{\mathcal{G}}$ which is impossible. So $\mathcal{T}$ is a tree.

By Lemma 3.34 we can assume that the stabilizer $G_{0}$ of a component $\mathcal{G}_{0} \in \mathcal{C}$ is finitely generated and contains the fixed finite set $K \subset G$. The group $G$ acts transitively on $A$ and so on $\mathcal{C}$. Then every element of $\mathcal{C}$ is stabilized by a subgroup conjugate to $G_{0}$. So in the graph $X=\mathcal{T} / G$ there is only one non-horospherical vertex $v_{0}=\mathcal{C} / G$ whose vertex group is $G_{0}$.

The set of horospheres on $T$ is $G$-finite (Lemma 3.17) so $X$ contains $n$ vertices of non horospherical type each representing the $G$-orbit of an horosphere $T(p)(p \in \mathcal{P})$. Every one of them is connected with $v_{0}$ by a unique edge. So every vertex group of horospherical type is $P_{i}$ and the edge groups are $Q_{i}=P_{i} \cap G_{0}(i=1, \ldots, n)$. The Theorem is proved.

3.5. Corollaries of Theorem A. Theorem A admits several immediate corollaries describing different type of finiteness properties of relatively hyperbolic groups.

Corollary 3.37. Let $G$ be a relatively hyperbolic group with respect to the system $P_{j}(j=1, \ldots, n)$. Then there exists an exhaustion

$$
G=\bigcup_{i \in I} G_{i},
$$

where $G_{i}$ is a finitely generated group which is relatively hyperbolic with respect to the system $P_{j} \cap G_{i}(j=1, \ldots, n)$.

Definition 3.38. A group $G$ is called relatively finitely generated with respect to a system $\mathfrak{P}$ of subgroups if it is generated by the system $\mathfrak{P}$ and a finite set $S$ generators.

Furthermore $G$ is relatively finitely presented with respect to $\mathfrak{P}$ if there are at most finitely many relations between the elements of $S$.

Corollary 3.39. Let $G$ be a group acting 3-discontinuously and 2-cocompactly on a compactum $T$. Then $G$ is relatively finitely generated with respect to the stabilizers of the parabolic points. 
Proof. Indeed by Theorem A the group $G$ is generated by a finitely generated subgroup $G_{0}$ and by the parabolic subgroups $H_{i}(i=1, \ldots, n)$. The Corollary follows.

Corollary 3.40. A group $G$ acting 3-discontinuously and 2-cocompactly on a compactum $T$ without parabolic points is finitely generated.

Remark. If in particular $G$ acts 3-discontinuously and 3-cocompactly on $T$ without isolated points then every point of $T$ is conical [17, Appendix]. So, by Corollary 3.40, $G$ is finitely generated in this case. By a direct argument one can now deduce that $G$ is word-hyperbolic [17, Appendix]. This provides a new proof of a theorem due to B. Bowditch [4].

Before we state the next corollary let us recall two more definitions of relative hyperbolicity valid for infinitely generated groups. The first one is due to B. Bowditch:

Definition 3.41 ([2]). A graph $\Gamma$ is called fine if there are at most finitely many simple arcs of a bounded length with fixed endpoints.

An action of a group $G$ on a graph $\Gamma$ is proper on the set of edges $\Gamma^{1}$ if the stabilizers of edges are finite, the action is called cofinite if $\left|\Gamma^{1} / G\right|<\infty$.

A group $G$ is called relatively hyperbolic with respect to a system of subgroups $\mathfrak{P}$ if $G$ acts non-parabolically on a connected fine hyperbolic graph $\Gamma$ cofinitely and properly on edges such that $\mathfrak{P}$ is a maximal set of non-conjugated infinite stabilizers of vertices.

The second definition is due to $\mathrm{D}$. Osin:

Definition 3.42 ([26, Definions $2.3,2.30,2.35]$ ). A group $G$ is relatively hyperbolic with respect to a finite system $\mathfrak{P}$ of subgroups of infinite index if it is finitely presented with respect to $\mathfrak{P}$ and the corresponding relative Cayley graph $\Gamma=\operatorname{Cay}(G, \mathfrak{P} \cup S)$ admits a linear relative Dehn function i.e. the relative area of a cycle in $\Gamma$ of length $\leq n$ is bounded by a linear function of $n$.

The main corollary of Theorem A is the following result establishing that all known definitions of the relative hyperbolicity valid for a group of any cardinality are equivalent to the existence of the star-graph of groups decomposition (1) of Theorem A. 
Non-finitely generated relatively hyperbolic groups and Floyd quasiconvexity

Theorem 3.1. The following conditions are equivalent for a group $G$.

1) Definition 1.1.

2) Bowditch's Definition 3.41.

3) Osin's Definition 3.42

4) $G$ admits the star-graph of groups decomposition (1) of Theorem A where the central vertex group $G_{0}$ is a finitely generated relatively hyperbolic group with respect to those edge groups $Q_{i}$ which are infinite.

Remark. In fact in [26] the finiteness of the system $\mathfrak{P}$ is not required. We need it to have the equivalence of the Osin's definition to all others definitions which all imply this assumption.

Proof. As it was mentioned in the Introduction modulo Theorem A and known facts the proof of the theorem goes according to the diagram $(*)$ from the Introduction. It remains only to show Propositions 3.43 and 3.47 whose proofs are given below.

Proposition 3.43. Let a group $G$ admit the graph $\mathfrak{G}$ of groups decomposition (1) of Theorem A. Suppose that the subgroup $G_{0}$ acts on a fine $\delta$-hyperbolic graph $\Gamma$ properly and cofinitely on the set of edges and the groups $Q_{i}$ are the stabilizers of $G_{0}$-non-equivalent vertices of $\Gamma$. Then there exists an action of $G$ on a fine $\delta$-hyperbolic graph $\Delta$ properly and cofinitely on $\Delta^{1}$ such that $P_{1}, P_{2}, \ldots$ are the stabilizers of $G$-non-equivalent vertices.

In particular, if $G_{0}$ is relatively hyperbolic in the sense of Bowditch with respect to the subgroups $Q_{i}$ then $G$ is relatively hyperbolic in the same sense with respect to the subgroups $P_{i}(i=1, \ldots, n)$.

Note that the statement above is more general than the implication 4) $\Longrightarrow 2$ ) as we do not need to assume that $G_{0}$ is finitely generated and that all the subgroups $P_{i}$ are infinite. The group $G$ is relatively hyperbolic with respect to those which are infinite.

Proof of the proposition. We will construct the graph $\Delta$ as the quotient of another graph $\widetilde{\Delta}$.

Let $\mathcal{T}$ be the universal covering tree of the graph $\mathfrak{G}$ and let $\tau: \mathcal{T} \rightarrow \mathfrak{G}$ be the covering map. For a vertex $v \in \tau^{-1}\left\{G_{0}\right\} \subset \mathcal{T}^{0}$ denote by $G_{0, v}$ its stabilizer St ${ }_{G} v$ in $G$ and by $\widetilde{\Gamma}_{v}$ a copy of $\Gamma$ on which the group $G_{0, v}$ acts. We can assume that all these copies are disjoint. 
There is a bijection between the $\mathcal{T}$-edges incident to $v$ and the vertices of $\widetilde{\Gamma}_{v}$. Using this bijection we can replace a small neighborhood of $v$ in $\mathcal{T}$ by $\widetilde{\Gamma}_{v}$ joining the $v$-endpoint of an edge with the corresponding vertex of $\widetilde{\Gamma}_{v}$. This implantation can be made $G$-equivariantly, so the obtained graph $\widetilde{\Delta}$ is connected and acted upon by $G$.

There are two kinds of the edges of $\widetilde{\Delta}$ : those of the graphs $\widetilde{\Gamma}_{v}$ and those of $\mathcal{T}$. Now we collapse all the edges of the second kind. Let $\Delta$ denote the resulting graph. Since the collapsing is $G$-equivariant there is an induces action $G \curvearrowright \Delta$ and the projection map $\pi: \widetilde{\Delta} \rightarrow \Delta$ is a $G$-equivariant morphism of graphs. Hence the graph $\Delta$ is connected too.

We have the diagram

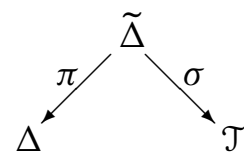

of $G$-equivariant graph morphisms where $\sigma$ collapses $\widetilde{\Gamma}_{v}$ to $v$.

Denote by $\Gamma_{v}$ the $\pi$-image of $\widetilde{\Gamma}_{v}$ is isomorphic to $\widetilde{\Gamma}_{v}$. Unlike the $\widetilde{\Gamma}_{v}$ 's the subgraphs $\Gamma_{v}$ of $\Delta$ are not disjoint.

Canonical lifting of the paths. For a vertex $w \in \Delta^{0}$ the subgraph $\pi^{-1} w$ of $\widetilde{\Delta}$ is either the star of a vertex $\tilde{w} \in \mathcal{T}^{0}$ such that $\tau \tilde{w}$ is one of the vertices $P_{i}$ or a vertex in some $\Gamma_{v}$. We say that $\tilde{w}$ is the central representative of $w$ in the first case.

Claim. For every locally injective path $\gamma: I \rightarrow \Delta$ between two vertices there exists a unique locally injective path

$$
\tilde{\gamma}: J \longrightarrow \tilde{\Delta}
$$

between the central representatives of the endpoints of $\gamma$ and a monotone map

$$
\iota: J \longrightarrow I
$$

such that

$$
\gamma \circ \iota=\tilde{\gamma} \circ \pi .
$$

If $\gamma$ is geodesic then $\tilde{\gamma}$ also is. 
Proof of the claim. Both existence and uniqueness follow from the fact that the $\pi$-preimages of vertices are connected subtrees and the maximal subpaths in $\Gamma_{v}$ 's lift uniquely to paths in $\widetilde{\Gamma}_{v}$. These lifted subpaths can be joined uniquely in the corresponding subtrees providing the lift of the whole path.

The statement about geodesic paths follows from the fact that every locally injective path in a tree is geodesic. The lemma is proved.

Verification of the properties of the action $G \curvearrowright \Delta$. By construction the action $G \curvearrowright \Delta^{1}$ is proper and cofinite.

Recall that a circuit in a graph $\Gamma$ is a subgraph homeomorphic to the circle $\mathbb{S}^{1}$. Every circuit of $\Delta$ is contained in a subgraph $\Gamma_{v}$ since otherwise its lift is a circuit in $\widetilde{\Delta}$ containing $\mathcal{T}$-edges so its $\sigma$-image is a non-trivial circuit in the tree $\mathcal{T}$ which is impossible. This implies that $\Delta$ is fine.

It remains to verify that $\Delta$ is $\delta$-hyperbolic provided that $\Gamma$ is. Let $\tau$ be a geodesic triangle in $\Delta$ and let $\tilde{\tau}$ denote the triangle in $\widetilde{\Delta}$ obtained from $\tau$ by the canonical lifting of the sides. By Lemma $\tilde{\tau}$ is geodesic in $\widetilde{\Delta}$ and $\sigma(\tau)$ is geodesic in $\mathcal{T}$. Thus $\tilde{\tau}$ actually consists in pieces that either $\mathcal{T}$-edges or geodesic bigons in subgraphs $\Gamma_{v}$ or (at most one) geodesic triangle in some $\Gamma_{v}$. Each this piece is $\delta$-thin by the hypothesis. The proposition is proved.

Remark. Proposition 3.43 gives a generic construction of non-finitely generated relatively hyperbolic groups. By Theorem A any relatively hyperbolic group can be constructed in this way.

Furthermore the implication 2) $\Longrightarrow 1$ ) of Proposition 3.43 and [16, Remark 9.1] imply that $G$ acts 3-discontinuously and 2-compactly on a compactum such that the $\left\{P_{i}: i=1, \ldots, n\right\}$ is a complete list of representatives of the stabilizers of parabolic points.

The rest of the subsection is devoted to the proof of the implication 2) $\Longrightarrow 3$ ). The argument is rather standard. However we did not find an adequate reference for non-finitely generated groups, so for the reader's convenience we provide it here. The argument below is motivated by [5].

In this section we consider graphs as 1-dimensional CW-complexes and cycles as non-oriented cycles.

Let $\Xi$ denote the graph with two vertices $P, Q$ and three edges $a, b, c$ that join $P$ with $Q$.

Every continuous map $\varphi: \Xi \rightarrow \Gamma$ determines three cycles in $\Gamma$; denote them by $\varphi_{a b}, \varphi_{b c}, \varphi_{c a}$. 
A non-negative function $\alpha$ on the set $\{$ cycles in $\Gamma\}$ is called a pseudo-area function on $\Gamma$ if

$$
\boldsymbol{\alpha}\left(\varphi_{a c}\right) \leqslant \boldsymbol{\alpha}\left(\varphi_{a b}\right)+\boldsymbol{\alpha}\left(\varphi_{b c}\right)
$$

for every continuous map $\varphi: \Xi \rightarrow \Gamma$.

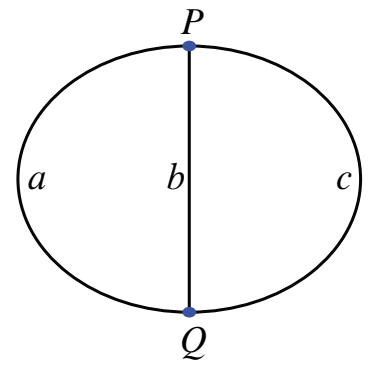

Let $\mathcal{C}$ be a set of circuits in a graph $\Gamma$. Denote by $\Gamma+\mathcal{C}$ the $\mathrm{CW}$-complex obtained from $\Gamma$ by attaching a 2 -cell to each circuit in $\mathcal{C}$.

For a locally injective map $\gamma: \mathbb{S}^{1} \rightarrow \Gamma$ denote by $\ell(\gamma)$ the natural length of $\gamma$ : this is the number of edges that $\gamma$ consecutively passes.

Lemma 3.44. Let a group $G$ act on a fine hyperbolic graph $\Gamma$ properly and cofinitely on the set of edges $\Gamma^{1}$ of $\Gamma$. Then there exists a $G$-finite set $\mathcal{C}$ of circuits in $\Gamma$ such that the complex $\Gamma+\mathcal{C}$ is simply-connected and for every $G$-invariant pseudo-area function $\boldsymbol{\alpha}$ on $\Gamma$ there exists a number $M$ such that $\boldsymbol{\alpha}(\gamma) \leqslant M \ell(\gamma)$ for every locally injective map $\gamma: \mathbb{S}^{1} \rightarrow \Gamma$.

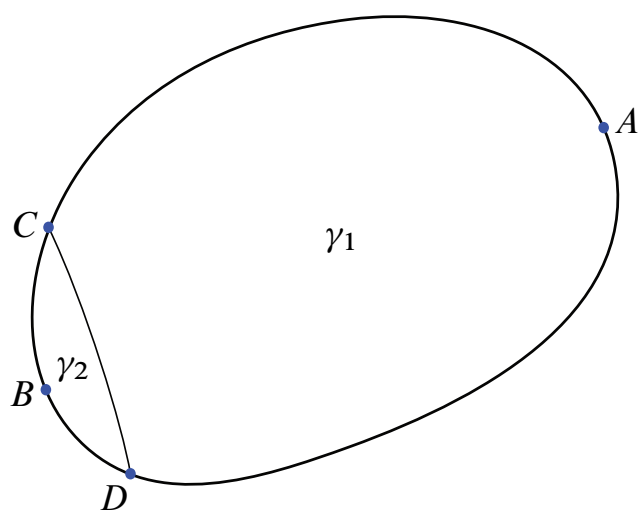

Proof. Consider a locally injective map $\gamma: \mathbb{S}^{1} \rightarrow \Gamma$. Choose points $A, B$ in the image of $\gamma$ such that $|A B|=\operatorname{diam} \operatorname{Im}(\gamma)$. We will show that $B$ belongs to a nongeodesic piece of $\gamma$ of a bounded length. 
Suppose that the $d$-neighborhood in $\gamma$ of the point $B$ is a geodesic segment. Then we have $|B C|=|B D|=d$ and $|C D|=2 d$. This implies

$$
|A B|+|C D| \geqslant d+\max \{|A C|+|B D|,|A D|+|B C|\} .
$$

Since our graph is hyperbolic, the value of $d$ is bounded by some constant $\delta[20$, 1.1.A].

Hence for $d=\delta+1$ the $\operatorname{arc} C B D$ is not geodesic. We join $C$ with $D$ by a geodesic segment $\lambda$. Let $\gamma_{1}=\lambda \cup \overparen{C A D}$ and $\gamma_{2}=\lambda \cup \overparen{C B D}$. The length of $\gamma_{2}$ is bounded by $4 \delta+3$.

Thus the cycle $\gamma_{2}$ possesses a tesselation by at most $4 \delta+3$ simple cycles of length at most $4 \delta+3$.

Since $\Gamma$ is fine and $\Gamma^{1} / G$ is finite, the set $\mathcal{C}=\{$ circuits of length $\leqslant 4 \delta+3\}$ is $G$-finite. As $\alpha$ is $G$-invariant there exists a constant $M$ such that $\alpha\left(\gamma_{2}\right) \leqslant M$. By the definition of pseudo-area $\alpha(\gamma) \leqslant \alpha\left(\gamma_{1}\right)+\alpha\left(\gamma_{2}\right)$. Since $\ell\left(\gamma_{1}\right) \leqslant \ell(\gamma)-1$ the desired inequality follows by induction on $l(\gamma)$. The lemma is proved.

Let $S$ denote a (finite) relative system of generators and $P_{i}(i \in I)$ be the system of all maximal parabolic subgroups of $G$. Recall that the set of vertices of the coned-off Cayley graph $\operatorname{Cof}(G, S \cup \mathcal{P})$ is $G \cup \mathcal{P}$ where $\mathcal{P}$ is the set of the parabolic points $p_{i}$ whose stabilizer is $P_{i}$. The set of edges is $E_{1} \cup E_{2}$ where $E_{1}$ is the set of edges of the absolute Cayley graph $\operatorname{Cay}(G, S)$ and $E_{2}$ is the set of edges joining every parabolic element in $P_{i}$ with the point $p_{i}$ fixed by $(i \in I)$; cf. [11].

Lemma 3.45. Let a group $G$ admit a cofinite and proper on edges action on a fine hyperbolic graph $\Gamma$. Then there exists a finite system $S$ of relative generators of $G$ such that the coned-off Cayley graph $\operatorname{Cof}(G, S \cup \mathcal{P})$ is also fine and hyperbolic and $G$ acts on it cofinitely and properly on edges, where $\mathcal{P}$ is a set of parabolic vertices of $\Gamma$.

Proof. We present a finite algorithm for passing from $\Gamma$ to $\operatorname{Cof}(G, S \cup \mathcal{P})$ by keeping all the above properties valid.

Consider first an intermediate graph $\Delta$ obtained from $\Gamma$ as follows. Set $\Delta^{0}=$ $\Gamma^{0} \sqcup G$. We call the vertices and the edges of $\Gamma$ blue and the elements of $G$ red. Denote by $F \subset \Gamma^{0}$ a fundamental set for the action $G \curvearrowright \Gamma^{0}$ containing one representative in each $G$-orbit of blue vertices. Join the vertex represented by the element 1 of $G$ with each vertex in $F$ by a red edge. Let $\eta$ denote this set of red edges. By applying to this new edges the elements of $G$ we obtain a $G$-invariant set $G \eta$ of red edges. Put $\Delta^{1}=\Gamma^{1} \cup G \eta$. 
To construct a new graph $\widetilde{\Delta}$ we have to add a finitely many orbits of new edges and eventually remove all the blue edges preserving the connectedness of the graph. We proceed as follows.

Let $e=(x, y) \in \Gamma^{1} \subset \Delta^{1}$ be a blue edge. If $\Delta \backslash G e$ is connected then put $\widetilde{\Delta}=\Delta \backslash G e$. Suppose that $\Delta \backslash G e$ is not connected and the endpoints $x$ and $y$ of a blue edge belong to different connected components of $\Delta \backslash G e$. We choose red vertices $x^{\prime}$ and $y^{\prime}$ adjacent to $x$ and $y$ respectively, join them with a new yellow edge $e^{\prime}$ and put $\widetilde{\Delta}=(\Delta \backslash G e) \cup G e^{\prime}$.

We need to show that adding or deleting the orbit of one edge keeps the properties of $\Gamma$ valid. We proceed by induction keeping the notation $\Delta$ for the graph of the previous step for which all the requested properties were true (at the beginning $\Delta=\Gamma$ ); and denote by $\widetilde{\Delta}$ the graph $\Delta \pm G e$ where $e$ is a red, a yellow or a blue edge (we add the red and the yellow edges and delete the blue ones). By construction at least one of the vertices of each new edge $e$ has finite stabilizer for $G \curvearrowright \Delta$, so the action on the new graph is still proper and cofinite. Since we first add a yellow edge and then delete the corresponding blue one the graph $\widetilde{\Delta}$ remains connected.

To prove the finess of the new graph we will use a result of [2] which we briefly state now for the completeness. A collection $\mathcal{L}$ of subgraphs of a graph is called edge-finite if for every edge $e$ the set $\left\{L \in \mathcal{L} \mid e \in \mathcal{L}^{1}\right\}$ is finite. We need the following

Lemma 3.46 ([2, Lemma 2.3]). Suppose that $K$ is a fine graph, and $\mathcal{A}$ is a collection of arcs of bounded length in $K$. Then the graph $K[\mathcal{A}]$ obtained by adding the edges joining the endpoints of the arcs in $\mathcal{A}$ is also fine.

By this lemma after the adding of the orbit of an edge to $\Delta$ the obtained graph remains fine. Indeed in the orbit $\mathcal{A}=\{g L: g \in G\}$ of a finite arc $L \subset \Delta$ the set $\left\{g \in G \mid e \in g L^{1}\right\}$ is finite. Otherwise, since the set $\Delta^{1} / G$ is finite, there would exist an edge of $L$ with infinite stabilizer.

It is obvious that the operation of deleting of an orbit of blue edges preserves the finess.

To check the hyperbolicity let us check that there is a map between the set of vertices $\Delta^{0}$ and $\widetilde{\Delta}^{0}$ of the graphs $\Delta$ and $\widetilde{\Delta}$ respectively which is at most $K$-bi-Lipschitz. Here $K=\max \{r+1,3\}$ and $r$ is the diameter of $F$. Indeed the map is at most 1-bi-Lipschitz at the beginning when we add the first orbit of red edges. Here the direct map is the identity on $\Gamma^{0}$ and the inverse map is the projection of $G$ to $\Gamma^{0}$ which does not increase the distances ( $G$ is not contained in $\left.\Gamma^{0}\right)$. On the next steps the direct map is still an isometry on $\Delta^{0}$. For the inverse 
map to return from $\widetilde{\Delta}$ to $\Delta$ we delete the orbit $G e$ of the edge $\{x, y\}$ where $y$ is red and $x \in F$ is a blue vertex. Note that $F$ contains a vertex $x_{0}$ already joined with 1 , so the distance between 1 and $x$ in $\Delta$ is at most $1+r$ asserting that the map is $(r+1)$-bi-Lipschitz.

In its turn adding of an orbit of yellow or blue edges is at most 3-bi-Lipschitz as we replace a path of length at most 3 by an edge. So on each step there is a quasi-isometry between the graphs $\Delta$ and $\widetilde{\Delta}$. Since the number of orbits is finite the process completes after a finitely many steps and the final graph is still hyperbolic [20].

To obtain a coned-off Cayley graph from the final graph $\widetilde{\Delta}$ it remains to remove each blue vertex $z$ having finite stabilizer (the blue vertices with infinite stabilizers will be the parabolic vertices of the coned-off Cayley graph). We also remove all the red edges incident to $z$ and join every two vertices adjacent to $z$ in $\widetilde{\Delta}$ by a yellow edge. By the same argument as above the obtained graph is connected, fine and hyperbolic, and the $G$-action on it is cofinite and proper on edges. Let $S$ denote the set of the elements of $G$ joined with the element 1 by yellow edges. It follows that the obtained graph is the coned-off Cayley graph $\operatorname{Cof}(G, S \cup \mathcal{P})$ where $\mathcal{P}$ is the set of parabolic vertices. The lemma is proved.

The following proposition finishes the proof of Theorem 3.1

Proposition 3.47. Let a group $G$ act on a fine hyperbolic graph $\Gamma$ properly and cofinitely on $\Gamma^{1}$. Then $G$ is relatively finitely presented with respect to any maximal set $\mathfrak{P}$ of non-conjugate infinite stabilizers of vertices for the action. Furthermore it admits a linear relative Dehn function.

Proof. By Lemma 3.45 there exists a finite set $S \subset G$ such that the coned-off Cayley graph $\operatorname{Cof}(G, S \cup \mathcal{P})$ is fine and hyperbolic. The group $G$ acts on it cofinitely and properly on edges.

By Lemma 3.44 there exists a simply connected complex

$$
\mathcal{W}=\operatorname{Cof}(G, S \cup \mathcal{P}) \cup \mathcal{C}
$$

where $\mathcal{C}$ is a $G$-finite set of circuits that bound 2-cells. For every such 2-cell $D$ whose vertices do not all belong to the star of one parabolic vertex we do the following surgery. Once the boundary $\partial D$ contains two consecutive red edges passing through a parabolic vertex $p$ we replace this pair of edges by one yellow edge and consider the component of $D$ not containing $p$. We cut in this way all parabolic vertices on $\partial D$ and obtain a 2-disk $D^{\prime}$ whose boundary lies in the relative Cayley graph. Proceeding similarly with all 2-cells of $\mathcal{W}$ we obtain a 2-complex 
$\mathcal{S}$ containing a $G$-finite set of 2-cells attached to circuits in the graph Cay $(G, S \cup$ $(\cup \mathfrak{P}))$. It follows that every singular disk in $\mathcal{S}$ whose boundary is not contained in the star of a parabolic vertex can be tessellated by a finite number of the 2-cells $D$ obtained above. So these cells give rise to a finite relative presentation for $G$ relatively to the parabolic subgroups $P_{i}(i \in I)$.

To estimate the Dehn function consider a circuit $\gamma$ in the complex $\mathcal{S}$. We can assume that no three consecutive vertices of $\gamma$ belong to a star of a parabolic vertex. We now replace every yellow boundary edge of $\gamma$ whose endpoints belong to the same coset of a parabolic subgroup $P_{i}$ by a pair of red edges in $\operatorname{Cof}(G, S \cup \mathcal{P})$ passing through its fixed point $p_{i}(i \in I)$. Let $\gamma^{\prime}$ be the obtained circuit in $\mathcal{W}$. We have $\boldsymbol{\alpha}(\gamma)=\boldsymbol{\alpha}^{\prime}\left(\gamma^{\prime}\right)$ and $\ell^{\prime}\left(\gamma^{\prime}\right) \leq 2 \ell(\gamma)$ where $\boldsymbol{\alpha}$ and $l$ (respectively $\boldsymbol{\alpha}^{\prime}$ and $l^{\prime}$ ) denote the relative area and the length of a circuit in the relative Cayley graph (respectively coned-off graph). By Lemmata 3.44 and 3.45, $\alpha^{\prime}\left(\gamma^{\prime}\right) \leq M \ell^{\prime}\left(\gamma^{\prime}\right)$ and so $\alpha(\gamma) \leq 2 M \ell(\gamma)$.

The proposition and Theorem 3.1 are proved.

\section{Floyd metrics and shortcut metrics}

From now on we will assume that $G$ is a finitely generated group acting 3-discontinuously and 2-cocompactly on a compactum $T$. Let us first recall few standard definitions concerning Floyd compactification (see [12], [24], [27], [16], and [17] for more details).

We will deal with abstract graphs even without assuming any group action (in particular it can be the Cayley graph or the entourage graph $\mathcal{G}$ considered in Section 3).

Let $\Gamma$ be a locally finite connected graph. For a finite path $\alpha: I \rightarrow \Gamma(I \subset \mathbb{Z})$ we define its length to be $|I|-1$. We denote by $d(\cdot, \cdot)$ the canonical shortest path distance function on $\Gamma$, and by $B(v, R)$ the ball at a vertex $v \in \Gamma^{0}$ of radius $R$.

Let

$$
f: \mathbb{N} \longrightarrow \mathbb{R}_{>0}
$$

be a function satisfying the following conditions: there exists $\lambda>0$ such that, for all $n \in \mathbb{N}$,

$$
\begin{gathered}
1<\frac{f(n)}{f(n+1)}<\lambda ; \\
\sum_{n \in \mathbb{N}} f(n)<+\infty .
\end{gathered}
$$


Define the Floyd length $L_{f, v}(\alpha)$ of a path $\alpha=\alpha(a, b) \subset \Gamma$ with respect to a vertex $v$ as follows:

$$
L_{f, v}(\alpha)=\sum_{i} f\left(d\left(v,\left\{x_{i}, x_{i+1}\right\}\right)\right)
$$

where $\alpha^{0}=\left\{x_{i}\right\}_{i}$ is the set of vertices of $\alpha$ (we assume $f(0):=f(1)$ to make it well-defined).

The Floyd metric $\delta_{f, v}$ is defined to be the corresponding shortest path metric:

$$
\delta_{f, v}(a, b)=\inf _{\alpha} L_{f, v}(\alpha),
$$

where the infimum is taken over all paths $\alpha$ between the vertices $a$ and $b$ in $\Gamma$. We denote by $\bar{\Gamma}_{f}$ the Cauchy completion of the metric space $\left(\Gamma, \delta_{f, v}\right)$ and call it Floyd completion. Let

$$
\partial_{f} \Gamma=\bar{\Gamma}_{f} \backslash \Gamma
$$

be its boundary, called Floyd boundary.

If $\Gamma$ is a Cayley graph $\mathrm{C} a(G, S)$ of a group $G$ with respect to a finite generating system $S$ we denote by $\bar{G}_{f}$ and by $\partial_{f} G$ the Floyd completion and the Floyd boundary respectively. Condition 1 above implies that the $G$-action extends to its Floyd completion $\bar{G}_{f}$ by homeomorphisms [24]. Therefore in this case for any $g \in G$ the Floyd metric $\delta_{g}$ is the $g$-shift of $\delta_{1}$ :

$$
\delta_{g}(x, y)=\delta_{1}\left(g^{-1} x, g^{-1} y\right), \quad x, y \in \bar{G}_{f}, g \in G,
$$

where 1 is the neutral element of $G$. Every two metrics $\delta_{g_{1}}$ and $\delta_{g_{2}}$ are bi-Lipschitz equivalent with a Lipshitz constant depending on $d\left(g_{1}, g_{2}\right)$. The same properties are valid for any locally finite, connected and $G$-finite graph $\Gamma\left(\left|\Gamma^{0} / G\right|<\infty\right)$.

Recall that a quasi-isometric map (or c-quasi-isometric map) $\varphi: X \rightarrow Y$ between two metric spaces $X$ and $Y$ is a correspondence such that

$$
\frac{1}{c} d_{X}(x, y)-c<d_{Y}(\varphi(x), \varphi(y)) \leq c d_{X}(x, y)+c,
$$

where $c$ is a uniform constant and $d_{X}, d_{Y}$ denote the metrics of $X$ and $Y$ respectively.

If in addition $d_{X}(\mathrm{id}, \psi \circ \varphi) \leq$ const for a (c-)quasi-isometric map $\psi: Y \rightarrow X$ we say that $\varphi$ is a $(c$-)quasi-isometry between $X$ and $Y$.

A $c$-quasi-isometric map $\varphi: I \rightarrow X$ is called $c$-quasigeodesic if $I$ is a convex subset of $\mathbb{Z}$ or $\mathbb{R}$. A quasigeodesic path $\gamma: I \rightarrow \Gamma$ defined on a half-infinite subset $I$ of $\mathbb{Z}$ is called (quasi-)geodesic ray; a (quasi-)geodesic path defined on the whole $\mathbb{Z}$ is called (quasi-)geodesic line.

The following lemma will be often used. 
Lemma 4.1 (Karlsson lemma). Let $\Gamma$ be a locally finite connected graph. Then for every $\varepsilon>0$ and every $c>0$, there exists a finite set $D$ such that $\delta_{v}$-length of every $c$-quasigeodesic $\gamma \subset \Gamma$ that does not meet $D$ is less than $\varepsilon$.

Remark. A. Karlsson [24] proved it for geodesics in the Cayley graphs of finitely generated groups. The proof of [24] does not use the group action and is also valid for quasigeodesics.

Consider now a set $S$ of paths of the form $\gamma:[0, n] \rightarrow \Gamma$ of unbounded length starting at the point $a=\alpha(0) \in \Gamma$. Every $\gamma \in S$ can be considered as an element of the product $\prod_{i \in I} B(a, i)$. Since $\Gamma$ is a locally finite graph the latter space is compact in the Tikhonov topology. So every infinite sequence $\left(\alpha_{n}\right)_{n} \subset S$ possesses a "limit path" $\delta:[0,+\infty) \rightarrow \Gamma$ whose initial segments are initial segments of $\alpha_{n}$.

The following lemma illustrates the properties of infinite quasigeodesics of $\Gamma$.

Lemma 4.2 ([17]). Let $\Gamma$ be a locally finite connected graph. Then the following statements are true:

1) every infinite ray

$$
r:[0,+\infty[\longrightarrow \Gamma
$$

converges to a point at the boundary,

$$
\lim _{n \rightarrow \infty} r(n)=p \in \partial_{f} \Gamma
$$

2) for every point $p \in \partial_{f} \Gamma$ and every $a \in \Gamma$ there exists a geodesic ray joining $a$ and $p$

3) every two distinct points in $\partial_{f} \Gamma$ can be joined by a geodesic line.

Let $\Gamma$ be a locally finite, connected graph on which a finitely generated group $G$ acts cocompactly. Besides the Floyd metrics the Floyd completion $\bar{\Gamma}_{f}$ possesses a set of shortcut pseudometrics which can be introduced as follows (see also [16] and [17]). Let $\omega$ be a closed $G$-invariant equivalence relation on $\bar{\Gamma}_{f}$. Then there is an induced $G$-action on the quotient space $\bar{\Gamma}_{f} / \omega$. A shortcut pseudometric $\bar{\delta}_{g}$ is the maximal element in the set of symmetric functions $\varrho: \bar{\Gamma}_{f} \times \bar{\Gamma}_{f} \rightarrow \mathbb{R}_{\geqslant 0}$ that vanish on $\omega$ and satisfy the triangle inequality, and the inequality $\varrho \leqslant \delta_{g}$. 
For $p, q \in \bar{\Gamma}_{f}$ the value $\bar{\delta}_{g}(p, q)$ is the infimum of the finite sums $\sum_{i=1}^{n} \delta_{g}\left(p_{i}, q_{i}\right)$ such that $p=p_{1}, q=q_{n}$ and $\left\langle q_{i}, p_{i+1}\right\rangle \in \omega(i=1, \ldots, n-1)$, cf. [8, p. 77]. Obviously, the shortcut pseudometric $\bar{\delta}_{g}$ is the $g$-shift of $\bar{\delta}_{1}$. The metrics $\bar{\delta}_{g_{1}}, \bar{\delta}_{g_{2}}$ are bi-Lipschitz equivalent for the same constant as for $\delta_{g_{1}}, \delta_{g_{2}}$.

The pseudometric $\bar{\delta}_{g}$ is constant on $\omega$-equivalent pairs of points of $\partial_{f} \Gamma$, so it induces a pseudometric on the quotient space $\bar{\Gamma}_{f} / \omega$. We denote this induced pseudometric by the same symbol $\bar{\delta}_{g}$.

Let $\Gamma$ be a connected, locally finite and $G$-finite graph. The graph $\mathcal{G}$ given by the discrete system $A=G\left(\boldsymbol{a}_{0}\right)\left(\boldsymbol{a}_{0} \in\right.$ Ent $\left.T\right)$ of entourages (see Definition 3.10 and Convention 3.12) is also locally finite, $G$-finite and connected (Lemma 3.11). So there exists a $c$-quasi-isometry $\varphi: \Gamma \rightarrow \mathcal{G}$. Let $f$ and $g$ be scaling functions satisfying both (1) and (2) as well as the condition

$$
\frac{g(n)}{f(c n)}<D, \quad n \in \mathbb{N},
$$

where $c$ is the above quasi-isometry constant. By [17, Lemma 2.5] the map $\varphi$ extends to a $G$-equivariant Lipshitz map between the Floyd completions $\bar{\Gamma}_{f}$ and $\overline{\mathcal{G}}_{g}$ of these graphs. We denote this map by the same letter $\varphi$. The following lemma is a direct consequence of the main result of [16]:

Lemma 4.3 (Floyd map). Let $G$ be a finitely generated group acting 3-discontinuously and 2-cocompactly on a compactum $T$. Then there exist $\mu \in] 0,1[$ and a continuous $G$-equivariant map

$$
F: \bar{\Gamma}_{f} \longrightarrow \widetilde{T}=A \sqcup T
$$

for the scaling function $f(n)=\mu^{n}$.

Furthermore for every vertex $v \in \Gamma^{0}$ the quantity $\bar{\delta}_{\boldsymbol{v}}(F(x), F(y))$ is a metric on $\widetilde{T}$ where $x, y \in \bar{\Gamma}_{f}$ and $v=\varphi(v)=F(v)$.

Proof. It follows from [16] that there exists $v \in] 0,1[$ and a continuous $G$-equivariant map $\mathcal{F}: \overline{\mathcal{G}}_{g} \rightarrow \widetilde{T}$ where $g(n)=v^{n}$.

Let $\varphi: \bar{\Gamma}_{f} \rightarrow \overline{\mathcal{G}}_{g}$ be the $G$-equivariant Lipshitz map described above where $f(n)=\mu^{n}$ and $\mu=v^{1 / c}$. Set $F=\mathcal{F} \circ \varphi$. The map $F$ transfers the pseudometric $\bar{\delta}_{v}$ on $\bar{\Gamma}_{f}$ to $\tilde{T}$ as follows:

$$
\bar{\delta}_{\boldsymbol{v}}(F(x), F(y))=\bar{\delta}_{v}(x, y), \quad \text { where } v=F(v), v \in \mathcal{C} a(G, S) .
$$

By [16] each $\bar{\delta}_{\boldsymbol{v}}$ is a metric on $\widetilde{T}$. The kernel of $F$ is the closed $G$-invariant equivalence relation on $\bar{\Gamma}_{f}$ such that $\bar{\delta}_{\boldsymbol{v}}(F(x), F(y))=0$. Indeed since $\bar{\delta}_{\boldsymbol{v}}$ is a metric on $\widetilde{T}$ the latter one yields $F(x)=F(y)\left(x, y \in \bar{G}_{f}\right)$. 
Remarks 4.4. 1) We will call the obtained metric $\bar{\delta}_{\boldsymbol{v}}(\boldsymbol{v}=F(v) \in A)$ on $\widetilde{T}$ shortcut (Floyd) metric.

2) Lemma 4.3 is in particularly true for any polynomial scalar function $f$. Moreover one can put $f=g$ as $f(c n) / f(n)=$ const in this case.

3) Since $\bar{\delta}_{g} \leq \delta_{g}$ the Karlsson Lemma 4.1 is also true when one replaces the Floyd $\delta_{v}$-length by the shortcut $\bar{\delta}_{g}$-length.

\section{Horospheres and tubes}

Let a finitely generated group $G$ act 3-discontinuously and 2-cocompactly on a compactum $T$. Then the graph of entourages $\mathcal{G}$ is connected (Lemma 3.11). We will use the graph distance $d_{A}$ on $\mathcal{G}$ as well as the set of shortcut metrics $\bar{\delta}_{\boldsymbol{v}}(\boldsymbol{v} \in \mathcal{G})$ on the compactified space $\tilde{T}=T \cup A$ coming from Lemma 4.3 where $A=\mathcal{G}^{0}$.

We obtain in this section several properties of tubes and horospheres which will be used later on.

Lemma 5.1. For any integer $k>1$ there exists a constant $v>0$ such that, for all $\boldsymbol{a}, \boldsymbol{c} \in \widetilde{T}=T \sqcup A$ and all $\boldsymbol{b} \in A$,

$$
\boldsymbol{a}-\boldsymbol{b}-\boldsymbol{c}(k) \Longrightarrow \bar{\delta}_{\boldsymbol{b}}(\boldsymbol{a}, \boldsymbol{c}) \geq v \text {. }
$$

Proof. For a fixed entourage $\boldsymbol{b} \in A$ let $C_{\boldsymbol{b}, k}$ denote the closure of the set $\{\{\boldsymbol{a}, \boldsymbol{c}\} \in$ $\widetilde{T} \times \widetilde{T}: \boldsymbol{a}-\boldsymbol{b}-\boldsymbol{c}(k)\}$ in $\widetilde{T}$. We first claim that the set $C_{\boldsymbol{b}, k}$ does not intersect the diagonal of $\widetilde{T} \times \widetilde{T}$. Suppose not and $(p, p) \in C_{\boldsymbol{b}, k} \cap \Delta^{2} \widetilde{T}$. Then there exist two infinite sequences $\left(\boldsymbol{a}_{n}\right)_{n}$ and $\left(\boldsymbol{c}_{n}\right)_{n}$ in $C_{\boldsymbol{b}, k}$ converging to $p$. By discreteness of $A$ we may suppose that $p \in T$. By Lemma 3.6 we have $\boldsymbol{a}_{n}-\boldsymbol{b}-\boldsymbol{c}_{n}(k)$. Let $U$ be a $\boldsymbol{b}$-small neighborhood of $p$. Then $U^{\prime}$ is $\boldsymbol{a}_{n}$-small and $\boldsymbol{c}_{n}$-small simultaneously for $n>n_{0}$. Hence $\operatorname{sh}_{b} a_{n} \cup \operatorname{sh}_{b} c_{n} \subset U$, and so $\Delta_{b}\left(\operatorname{sh}_{b} a_{n}, \operatorname{sh}_{b} c_{n}\right) \leq 1$ which is impossible. It follows that $C_{\boldsymbol{b}, k} \cap \Delta^{2} \widetilde{T}=\emptyset$.

Since $C_{\boldsymbol{b}, k}$ is a closed subset of $\widetilde{T} \times \widetilde{T}$, and $\bar{\delta}_{\boldsymbol{b}}$ is a metric on $\widetilde{T}$, there exists a constant $v(\boldsymbol{b})>0$ such that $\bar{\delta}_{\boldsymbol{b}}(\boldsymbol{a}, \boldsymbol{c}) \geq v(\boldsymbol{b})$ on $C_{\boldsymbol{b}, k}$. Thus our statement holds for the set $C_{\boldsymbol{b}, k}$ of entourages separated by the fixed entourage $\boldsymbol{b}$.

We have $A=G\left(\boldsymbol{a}_{0}\right)$. If now $\boldsymbol{a}-\boldsymbol{b}-\boldsymbol{c}(k)$ then there exists $g \in G$ such that $\boldsymbol{b}=g\left(\boldsymbol{a}_{0}\right)$, so $g^{-1} \boldsymbol{a}-\boldsymbol{a}_{0}-g^{-1} \boldsymbol{c}(k)$. Thus $\bar{\delta}_{\boldsymbol{b}}(\boldsymbol{a}, \boldsymbol{c})=\bar{\delta}_{\boldsymbol{a}_{0}}\left(g^{-1}(\boldsymbol{a}), g^{-1}(\boldsymbol{c})\right) \geq v$, where $v=v\left(\boldsymbol{a}_{0}\right)$ is the above constant for $\boldsymbol{a}_{0}$. The lemma is proved.

The following lemmas give a local description of $C$-quasigeodesics around tubes and horospheres. 
Lemma 5.2. There exists a constant $D>0$ such that, for every $C$-quasigeodesic $\gamma=\gamma(\boldsymbol{a}, \boldsymbol{c})$ in $\mathcal{G}$ with the endpoints $\boldsymbol{a}, \boldsymbol{c}$ and all $\boldsymbol{b} \in \Psi_{k}(\boldsymbol{a}, \boldsymbol{c})$,

$$
d_{A}(\boldsymbol{b}, \gamma) \leq D,
$$

where $\Psi_{k}(\boldsymbol{a}, \boldsymbol{c})=\{\boldsymbol{b} \in A: \boldsymbol{a}-\boldsymbol{b}-\boldsymbol{c}(k)\}$.

Proof. By Lemma 5.1 we have $\bar{\delta}_{\boldsymbol{b}}(\boldsymbol{a}, \boldsymbol{c}) \geq v$, and so the Floyd length $L_{f, \boldsymbol{b}}(\gamma)$ of $\gamma$ is at least $\nu$. By Karlsson Lemma 4.1 (see also Remark 4.4.3)) there exists a constant $D>0$ such that $\gamma \cap B(\boldsymbol{b}, D) \neq \emptyset$ for the $d_{A}$-ball $B(\boldsymbol{b}, D)$ in $\mathcal{G}$ centered at $\boldsymbol{b}$ with the radius $D$. The lemma is proved.

Lemma 5.3. 1) For any $C>0$ and $E \geq 0$ there exists $L>0$ such that for any parabolic point $p \in T$ and any $C$-quasigeodesic $\gamma:[0,1] \rightarrow \mathcal{G}$ one has

$$
d_{A}(\gamma(1), T(p)) \leq E \Longrightarrow d_{A}\left(\gamma, \Pi_{p}(\gamma(0))\right) \leq L
$$

2) There exists a constant $D>0$ such that for any parabolic point $p \in T$ and any $C$-quasigeodesic $\gamma:[0, \infty[\rightarrow \mathcal{G}$ one has

$$
\lim _{n \rightarrow \infty} \gamma(n)=p \Longrightarrow d_{A}\left(\gamma, \Pi_{p}(\gamma(0))\right) \leq D
$$

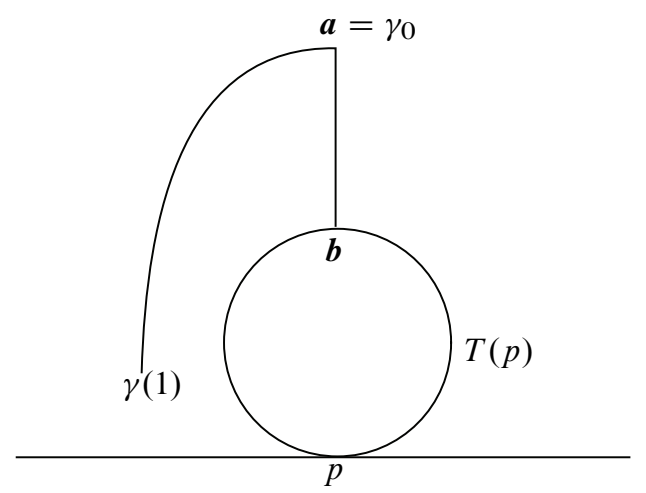

Figure 3. Quasigeodesics around horospheres. 
Proof. 1) Suppose not, then there exist constants $C$ and $E$ such that for any $n$ there exist a parabolic point $p_{n}$ and a $C$-quasigeodesic $\gamma_{n}:[0,1] \rightarrow \mathcal{G}$ such that $d_{A}\left(\gamma_{n}(1), T\left(p_{n}\right)\right) \leq E$ and $d_{A}\left(\gamma_{n}, \Pi_{p_{n}}\left(\gamma_{n}(0)\right)\right)>n$ for all $n \in \mathbb{N}$. By Lemma 3.17 there are at most finitely many $G$-non-equivalent parabolic points. So we may assume that $p=p_{n}$ and let $\boldsymbol{b}_{n} \in \Pi_{p}\left(\gamma_{n}(0)\right)$. By the same lemma the group $\operatorname{Stab}_{G} p$ acts cofinitely on $T(p)$ so we may also suppose that $\boldsymbol{b}=\boldsymbol{b}_{n}$.

Since $d_{A}\left(\gamma_{n}(1), \boldsymbol{b}\right)$ is unbounded the set $\left\{\gamma_{n}(1)\right\}_{n}$ is infinite. As $d_{A}\left(\gamma_{n}(1)\right.$, $T(p)) \leq E$ by Lemma 3.18 up to passing to a subsequence we get $\gamma_{n}(1) \rightarrow p$. Denote $\boldsymbol{a}_{n}=\gamma_{n}(0)$ and $\boldsymbol{c}_{n}=\gamma_{n}(1)$. For all $n$ we have $\boldsymbol{a}_{n} \neq \boldsymbol{b}$ so $\boldsymbol{a}_{n} \notin T(p)$ and $\boldsymbol{a}_{n}-\boldsymbol{b}-p$. By Lemma 3.6 we obtain $\boldsymbol{a}_{n}-\boldsymbol{b}-\boldsymbol{c}_{n}\left(n>n_{0}\right)$. Thus Lemma 5.2 implies that $d_{A}\left(\boldsymbol{b}, \gamma_{n}\right) \leq D$ which is a contradiction. Statement 1) is proved.

2) We have $\lim _{n \rightarrow \infty}\left(\gamma(n)=c_{n}\right)=p$ and without lost of generality we can suppose that $\boldsymbol{a}=\gamma(0) \notin T(p)$. Then arguing similarly we obtain $\boldsymbol{a}-\boldsymbol{b}-\boldsymbol{c}_{n}\left(n>n_{0}\right)$ where $\boldsymbol{b}=\Pi_{p}(\boldsymbol{a})$. From Lemma 5.2 we have $d_{A}(\boldsymbol{b}, \gamma) \leq D$.

The following lemma is a generalization of the previous one to the geodesics with variable endpoints:

Lemma 5.4. 1) For any $C>0$ and $E \geq 0$ there exists $M>0$ such that for any parabolic point $p \in T$ and any $C$-quasigeodesic $\gamma:[-1,1] \rightarrow \mathcal{G}$ one has

$$
d_{A}(\{\gamma(-1), \gamma(1)\}, T(p)) \leq E \Longrightarrow d_{A}\left(\gamma(0), \Pi_{p}(\gamma(0))\right) \leq M .
$$

2) There exists a constant $D>0$ such that for any parabolic point $p \in T$ and any $C$-quasigeodesic $\gamma:[-\infty,+\infty[\rightarrow \mathcal{G}$ one has

$$
\lim _{n \rightarrow \pm \infty} \gamma(n)=p \Longrightarrow d_{A}\left(\gamma(0), \Pi_{p}(\gamma(0))\right) \leq D .
$$

Proof. 1) As before using the finiteness of $G$-non-equivalent parabolic points, we fix a parabolic point $p$. Let $\gamma_{-}=\gamma([-1,0])$, and $\gamma_{+}=\gamma([0,1])$. If $\boldsymbol{a}=$ $\gamma(0) \notin T(p)$ and $\boldsymbol{b}=\Pi_{p}(\boldsymbol{a})$ then by the statement 1) of Lemma 5.3 we have $d_{A}\left(\gamma_{ \pm}, \boldsymbol{b}\right) \leq L$. Let $z \in \gamma_{+}$and $\boldsymbol{y} \in \gamma_{-}$be the points realizing these distances. Since there is a path from $z$ to $\boldsymbol{y}$ through $\boldsymbol{b}$ of length $2 L$, the length $l(\gamma(z, y)))$ of the

$C$-quasigeodesic $\gamma(z, y)$ between $z$ and $\boldsymbol{y}$ is at most $2 L(C+1)$. So at least for one of these entourages, e.g. $z$, we have $l(\gamma(\boldsymbol{a}, \boldsymbol{z})) \leq L(C+1)$. By the triangle inequality we obtain $d_{A}(\boldsymbol{a}, \boldsymbol{b}) \leq M=L(C+2)$.

The same argument and Lemma 5.3.2) imply statement 2). 
The following Corollary establishes the uniform quasiconvexity of all horospheres and the quasiconvexity (simple) of every parabolic subgroup (see also [15] and [17]).

Corollary 5.5. Suppose $G$ acts 3-discontinuously and 2-cocompactly T. Then there exists $M>0$ such that for every $p \in \mathcal{P}$ the horosphere $T(p)$ is a $M$-quasiconvex subset of $A$.

Furthermore for every $p \in \mathcal{P}$ there exists a constant $D_{p}$ such that the parabolic subgroup $H_{p}=\operatorname{Stab}_{G} p$ is $D_{p}$-quasiconvex.

Proof. Suppose first that $\gamma \subset A$ is a $C$-quasigeodesic with $\partial \gamma \subset T(p)$ for some $p \in \mathcal{P}$. By Lemma 5.4.1 for $E=0$ there exists a uniform constant $M>0$ such that $\gamma \subset N_{M}(T(p))$, where $N_{M}(\cdot)$ denotes the $M$-neighborhood with respect to the distance $d_{A}$.

To prove the second part note that since $G$ is finitely generated it is enough to prove it for the graph $\mathcal{G}$ quasi-isometric to the Cayley graph. By Lemma 3.17 for every $p \in \mathcal{P}$ the set $T(p) / H_{p}$ is finite where $H_{p}=\operatorname{Stab}_{G} p$. So there exists a constant $E=E(p)$ such that $H \subset N_{E}\left(T_{p}\right)$ and $T(p) \subset N_{E}(H)$. So if $\gamma \subset A$ is a $C$-quasigeodesic with $\partial \gamma \subset H$ then $d_{A}(\partial \gamma, T(p)) \leq E$. Then again by Lemma 5.4.1) there exists a constant $M=M(p)$ such that $\gamma \subset N_{M}(T(p))$. So $\gamma \subset N_{D_{p}}(H)$ where $D_{p}=M+E$.

Remark. Above Lemmata 5.3 and 5.4 are close to some lemmas contained in our work [17] where the horospheres were defined without using the entourages. We need the above results in terms of entourages to apply them in the further argument where the language of entourages is crucial.

By Proposition 3.27.3b) we have that for every $d>0$ there exists $e=e(d)>0$ such that, $p, q \in \mathcal{P}$,

$$
\operatorname{diam}\left(N_{d}\left(T(p) \cap N_{d}(T(q))\right) \leq e .\right.
$$

Definition 5.6. Let $\gamma \subset \widetilde{T}$ be a $C$-quasigeodesic. We call an entourage $v \in \gamma$ $d$-horospherical if there exist parts $[\boldsymbol{v}, \boldsymbol{c}]$ and $[\boldsymbol{a}, \boldsymbol{v}]$ of $\gamma$ of length greater than the constant $e$ and which are contained in a $d$-neighborhood $N_{d}(T(p))$ of a horosphere $T(p)$.

The entourage $v \in \gamma$ is called non-horospherical in the opposite case.

Remark. By 4 we can suppose that the parabolic point $p$ with respect to which the (non)-horosphericity is considered is unique. 
Lemma 5.7. Let $\gamma=\gamma(\boldsymbol{a}, \boldsymbol{c})$ be a c-quasigeodesic. Suppose that $P=P(\boldsymbol{a}, \boldsymbol{c})$ is a non-refinable tube having the same ending vertices $\boldsymbol{a}$ and $\boldsymbol{c}$ as $\gamma$. For every sufficiently large $d>0$ there exists a constant $E>0$ such that $d_{A}(g, P) \leq E$ for every $d$-non-horospherical point $\boldsymbol{g} \in \gamma$.

Proof. Note that the non-refinable tube $P(\boldsymbol{a}, \boldsymbol{c})$ exists by Proposition 3.25. By Lemma 5.2 there exists $D>0$ such that for every $\boldsymbol{p}_{i} \in P$ we have $d_{A}\left(\boldsymbol{p}_{i}, \gamma\right) \leq$ $D(i=1, \ldots, m)$. So let us fix a non-horospherical entourage $\boldsymbol{g} \in \gamma$, and let $\boldsymbol{g}_{i} \in \gamma$ be such that $d_{A}\left(\boldsymbol{p}_{i}, \gamma\right)=d_{A}\left(\boldsymbol{p}_{i}, \boldsymbol{g}_{i}\right)(i=0, \ldots, m)$. Let us also assume that $\boldsymbol{g} \in \gamma\left(\boldsymbol{g}_{i}, \boldsymbol{g}_{i+1}\right)$ where $\gamma\left(\boldsymbol{g}_{i}, \boldsymbol{g}_{i+1}\right)$ denotes the part of $\gamma$ between $\boldsymbol{g}_{i}$ and $\boldsymbol{g}_{i+1}$.

By Corollary 3.29 there exists a constant $C>0$ such that if $d_{A}\left(\boldsymbol{p}_{i}, \boldsymbol{p}_{i+1}\right)>C$ then the pair $\left\{\boldsymbol{p}_{i}, \boldsymbol{p}_{i+1}\right\}$ is contained in a horosphere $T(p)$. In this case we have $\left\{\boldsymbol{g}_{i}, \boldsymbol{g}_{i+1}\right\} \subset N_{D}(T(p))$ and by Lemma 5.4 we have that $\gamma\left(\boldsymbol{g}_{i}, \boldsymbol{g}_{i+1}\right) \subset N_{L}(T(p))$ for some $L=L(D)>0$. Let $d$ be any number bigger than $L$. If $g$ is $d$-nonhorospherical then, by Definition 5.6, $d_{A}\left(\boldsymbol{g}, \boldsymbol{g}_{i}\right)$ or $d_{A}\left(\boldsymbol{g}, \boldsymbol{g}_{i+1}\right)$ is less than $e$. Thus $d_{A}(g, P) \leq e+d$.

If now $d_{A}\left(\boldsymbol{p}_{i}, \boldsymbol{p}_{i+1}\right) \leq C$ then $d_{A}\left(\boldsymbol{g}_{i}, \boldsymbol{g}_{i+1}\right) \leq c(C+2 D)+c$. So $d_{A}(\boldsymbol{g}, P) \leq$ $d_{A}\left(\boldsymbol{g}_{i}, \boldsymbol{g}\right)+D \leq c(C+2 D)+c+D$.

Put $E=\max \{e+d, c(C+2 D)+c+D\}$. The lemma is proved.

Remark. The constants $d$ and $e$ depend on the constants $D, C$ and $L=L(D)$ given respectively by Lemma 5.2, Corollary 3.29, and Lemma 5.4.

\section{Tight curves in $\mathcal{G}$}

Let a finitely generated group $G$ act 3-discontinuously and 2-cocompactly on a compactum $T$. For a parabolic point $p$ we denote by $N(T(p))$ a neighborhood of the horosphere $T(p)$ in the graph $\mathcal{G}$ (see Section 3.2). The notation diam $(\cdot)$ is used for the diameter of a set with respect to the distance $d_{A}$ and $|\cdot|$ stands for the length of a curve. We denote by $c^{-1}(n)$ the linear function $\frac{n}{c}-c$ for some constant $c>0$.

Definition 6.1. For positive integers $l$ and $c$, a curve $\gamma: I \rightarrow \mathcal{G}$ is called $(l, c)$-tight (or just tight when the values of $l$ and $c$ are fixed) if for every $J \subset I$ the following conditions hold:

1) $|J| \leq\left. l \Longrightarrow \gamma\right|_{J}$ is a $c$-quasigeodesic;

2) if $|\gamma(J) \cap N(T(p))|>l$ for some $p \in \mathcal{P}$ then $\operatorname{diam}(\gamma(\partial J))>c^{-1}(l)$. 
The rest of the section is devoted to the proof of the following theorem describing the non-horospherical points (see Definition 5.6) of tight curves.

Theorem B. For every $c>0$ and $d>0$ there exist positive constants $l_{0}, w_{0}, c_{0}$ such that for all $l \geq l_{0}$ and every $(l, c)$-tight curve $\gamma \subset \mathcal{G}$ there exists a $c_{0}$-quasigeodesic $\alpha \subset A$ such that every $d$-non-horospherical vertex of $\gamma$ belongs to the $w_{0}$-neighborhood $N_{w_{0}}(\alpha)$ of $\alpha$.

The following three lemmas are close to the results of the previous section. We use below the notation $\operatorname{diam}_{\bar{\delta}_{v}}$ for the diameter of a set with respect to the shortcut metric $\bar{\delta}_{\boldsymbol{v}}(\boldsymbol{v} \in A)$ on $\tilde{T}$ (see Lemma 4.3)

Lemma 6.2. There exist positive constants $\rho$ and $d$ such that for every $c$-quasigeodesic $\gamma: I \rightarrow \mathcal{G}$ of non-zero length and a $d$-non-horospherical point $\gamma(0) \in \mathcal{G}$ one has

$$
\operatorname{diam}_{\bar{\delta}_{\gamma(0)}}(\gamma(\partial I))>\rho .
$$

Proof. Let us first prove that there exists a constant $r>0$ such that for some $\rho=\rho(r)$ we have

$$
d_{A}(\gamma(0), \gamma(\partial I))>r \Longrightarrow \bar{\delta}_{\gamma(0)}(\gamma(\partial I))>\rho .
$$

Suppose the converse. Then for every $d>0$ there exists a sequence of quasigeodesics $\gamma_{n}$ such that $d_{A}\left(\gamma_{n}(0), \gamma_{n}(\partial I)\right) \rightarrow+\infty$ and $\bar{\delta}_{\gamma_{n}(0)}(\gamma(\partial I)) \rightarrow 0$ where $\gamma_{n}(0)$ is a $d$-non-horospherical point of $\gamma_{n}$.

Up to choosing a subsequence we may suppose that the sequence $\left(\gamma_{n}\right)_{n}$ converges in the Tikhonov topology to a $c$-quasigeodesic $\gamma: \mathbb{Z} \rightarrow \mathcal{G}$ such that $\lim _{n \rightarrow \pm \infty} \gamma(n)=p \in T$. Then $\gamma$ is a horocycle at $p$ and by [17, Lemma 3.6] the point $p$ is parabolic. By Lemma 5.4.2) for every $i \in \mathbb{Z}$ the distance $d_{A}\left(\gamma_{n}(i), T(p)\right)$ is uniformly bounded by a constant $D>0$. So the points $\gamma_{n}(0)$ are $D$-horospherical for sufficiently large $n$. The obtained contradiction proves $(*)$.

We are left now with the case when $d_{A}(\gamma(0), \gamma(\partial I)) \leq r$ where the constant $r$ satisfies $(*)$. Suppose first that the distance between $\gamma(0)$ and both endpoints of $\gamma(\partial I)$ is less than $r$. By translating $\gamma(0)$ to a fixed basepoint $v \in A$ we obtain that $\gamma$ is contained in a finite ball $B(v, r+c(r))$. Then the $\bar{\delta}_{\boldsymbol{v}}$-length of $\gamma$ is uniformly bounded from below. If the distance between $\gamma(0)$ and only one of its endpoints is bigger than $r$ then the $\bar{\delta}$-length of $\gamma$ is still bounded from below.

Denoting by $\rho$ the minimum among all of these constants we obtain the lemma. 
Remark. Above we have used Lemma 3.6 from [17] stated there for the Cayley graphs. Since our graph $\mathcal{G}$ is quasi-isometric to the Cayley graph this result can be applied.

Recall that $A=G\left(\boldsymbol{a}_{0}\right)$ is the vertex set of the graph $\mathcal{G}$. Using a "refining" procedure we will now introduce a new graph $\mathcal{G}^{*}$ whose vertex set $A^{*}$ satisfies some additional conditions.

From now on we fix the constant $d$ and $\rho=\rho(d)$ coming from Lemma 6.2 and an integer $k>3$ which will be used in the betweenness relation below. Let $\delta$ be a number such that

$$
0<\delta<\frac{\rho}{k+2}
$$

Definition of the set $A^{*}$. For every $v \in A$ denote by $\boldsymbol{v}^{*}$ the entourage $\{\{x, y\} \in$ $\left.S^{2} T: \bar{\delta}_{v}(x, y)<\delta\right\}$.

It follows from the following lemma that the compactifying topology on $T$ coming from the graphs $A^{*}$ and $A$ is the same.

Lemma 6.3. For all $p \in T, \boldsymbol{a}_{n} \rightarrow p$ if and only if $\boldsymbol{a}_{n}^{*} \rightarrow p$.

Proof. Suppose first that $\boldsymbol{a}_{n} \rightarrow p$ and $\boldsymbol{a}_{n}^{*} \not \rightarrow p$. Then there exists a neighborhood $U_{p}$ of the point $p$ such that $U_{p}^{\prime}$ is not $\boldsymbol{a}_{n}^{*}$-small for $n>n_{0}$. So there exist $\boldsymbol{x}_{n}, \boldsymbol{y}_{n} \in$ $U_{p}^{\prime}$ such that $\bar{\delta}_{\boldsymbol{a}_{n}}\left(\boldsymbol{x}_{n}, \boldsymbol{y}_{n}\right)>\delta$. It follows that up to subsequences we have $\boldsymbol{x}_{n} \rightarrow$ $x \in T, y_{n} \rightarrow y \in T(n \rightarrow \infty)$ and $x \neq y \neq p \neq x$. Let $U_{x}$ and $U_{y}$ be closed neighborhoods of $x$ and $y$ such that $U_{p} \cap U_{x} \cap U_{y}=\emptyset$.

Let $H\left(U_{x, y}\right) \subset \mathcal{G}$ denote the set of geodesics whose endpoints are situated in $U_{x, y}=U_{x} \sqcup U_{y}$. By [17, Main Lemma] $\overline{H\left(U_{x, y}\right)} \cap T=\overline{U_{x, y}} \cap T$ where $\bar{U}_{x, y}$ means the closure of $U_{x, y}$ in $\widetilde{T}=A \sqcup$ Ent $T$. It follows that the geodesics $\gamma_{n}\left(\boldsymbol{x}_{n}, \boldsymbol{y}_{n}\right) \subset \mathcal{G}$ between $\boldsymbol{x}_{n}$ and $\boldsymbol{y}_{n}$ do not intersect a neighborhood $V_{p} \subset U_{p}$ of $p\left(n>n_{0}\right)$. Since $\boldsymbol{a}_{n} \rightarrow p$ we have $d_{A}\left(\boldsymbol{a}_{n}, \gamma_{n}\right) \rightarrow \infty$. By Karlsson Lemma 4.1 (see also Remark 4.4.3)) we obtain that $\bar{\delta}_{\boldsymbol{a}_{n}}\left(\boldsymbol{x}_{n}, \boldsymbol{y}_{n}\right)<\delta\left(n>n_{0}\right)$ which is a contradiction.

Suppose now $\boldsymbol{a}_{n}^{*} \rightarrow p$ and $\boldsymbol{a}_{n} \not \rightarrow p$. Then up to a subsequence we have $\boldsymbol{a}_{n} \rightarrow q$ $\neq p$. Let $U_{p}$ be a neighborhood of $p$ such that $U_{p}^{\prime}$ is $\boldsymbol{a}_{n}^{*}$-small $\left(n>n_{0}\right)$. We have $d_{A}\left(\boldsymbol{a}_{n}, U_{p}\right) \rightarrow+\infty$. Then by Karlsson Lemma, for all $x, y \in U_{p}, \bar{\delta}_{\boldsymbol{a}_{n}}(x, y)<$ $\delta\left(n>n_{0}\right)$. So $U_{p}$ and $U_{p}^{\prime}$ are both $\boldsymbol{a}_{n}^{*}$-small $\left(n>n_{0}\right)$ which is impossible (see (1) of Section 3.1). 
The need of the graph $A^{*}$ is explained by the following lemma.

Lemma 6.4. There exists constant $w>0$ such that for every quasigeodesic

$$
\gamma: I \longrightarrow \mathcal{G}
$$

containing three vertices $\boldsymbol{a}, \boldsymbol{b}, \boldsymbol{c} \in A$,

$\boldsymbol{b}$ is $d$-non-horospherical $\wedge d_{A}(\boldsymbol{b},\{\boldsymbol{a}, \boldsymbol{c}\})>w \Longrightarrow \boldsymbol{a}^{*}-\boldsymbol{b}^{*}-\boldsymbol{c}^{*}(k)$.

Proof. Suppose not and there are sequences $\boldsymbol{a}_{n}, \boldsymbol{c}_{n}$ and $\boldsymbol{b}_{n}$ such that $\boldsymbol{b}_{n}$ is $d$-nonhorospherical, $d_{A}\left(\boldsymbol{b}_{n},\left\{\boldsymbol{a}_{n}, \boldsymbol{c}_{n}\right\}\right) \rightarrow \infty$ and $\boldsymbol{a}_{n}^{*}-\boldsymbol{b}_{n}^{*}-\boldsymbol{c}_{n}^{*}(k)$ is not true. Since $A$ is $G$-finite we can suppose that $\boldsymbol{b}_{n}=\boldsymbol{b}$. Up to a subsequence we have $\boldsymbol{a}_{n} \rightarrow p$, $\boldsymbol{c}_{n} \rightarrow q$. Let $\gamma_{n}=\gamma_{n}\left(\boldsymbol{a}_{n}, \boldsymbol{c}_{n}\right) \subset \mathcal{G}$ be a geodesic between $\boldsymbol{a}_{n}$ and $\boldsymbol{c}_{n}$. Since $\boldsymbol{b}$ is non-horospherical we have by Lemma 6.2 that $\bar{\delta}_{\boldsymbol{b}}(p, q)>\rho$, hence $p \neq q$.

Let $U_{p}$ and $U_{q}$ be disjoint $\boldsymbol{b}^{*}$-small neighborhoods of $p$ and $q$ respectively. So $\bar{\delta}_{\boldsymbol{b}}(U, V)>\rho-2 \delta$, and $(* *)$ yields $\bar{\delta}_{\boldsymbol{b}}\left(U_{p}, U_{q}\right) \geq \rho-2 \delta>k \cdot \delta$. We obtain $\Delta_{\boldsymbol{b}^{*}}\left(U_{p}, U_{q}\right)>k$. By Lemma 6.3 we also have $\boldsymbol{a}_{n}^{*} \rightarrow p$ and $\boldsymbol{c}_{n}^{*} \rightarrow q$. So $U_{p}^{\prime}$ and $U_{q}^{\prime}$ are $\boldsymbol{a}_{n}^{*}$-small and $\boldsymbol{c}_{n}^{*}$-small respectively $\left(n>n_{0}\right)$. Hence $U_{p} \supset \operatorname{sh}_{\boldsymbol{b}^{*}} \boldsymbol{a}_{n}^{*}$ and $U_{q} \supset \operatorname{sh}_{\boldsymbol{b}^{*}} \boldsymbol{c}_{n}^{*}$. It follows that $\Delta_{\boldsymbol{b}^{*}}\left(\operatorname{sh}_{\boldsymbol{b}^{*}} \boldsymbol{a}_{n}^{*}, \operatorname{sh}_{\boldsymbol{b}^{*}} \boldsymbol{c}_{n}^{*}\right) \geq\left(\Delta_{\boldsymbol{b}^{*}}\left(U_{p}, U_{q}\right)>k\right.$. Therefore $\boldsymbol{a}_{n}^{*}-\boldsymbol{b}^{*}-\boldsymbol{c}_{n}^{*}(k)$ which is a contradiction.

Lemma 6.5. For every $d>0$ there exists a constant $l_{0}$ such that for every parabolic point $p$, and all entourages $\boldsymbol{b}, \boldsymbol{c}, \boldsymbol{d} \in N_{d}(T(p))$, and $\boldsymbol{a} \in A$, for all $l>l_{0}$ one has

$$
d_{A}(\boldsymbol{b}, \boldsymbol{c})>l \wedge d_{A}(\boldsymbol{b}, \boldsymbol{d})>l \wedge \boldsymbol{a}^{*}-\boldsymbol{b}^{*}-\boldsymbol{c}^{*}(k) \Longrightarrow \boldsymbol{a}^{*}-\boldsymbol{b}^{*}-\boldsymbol{d}^{*}(k-1) .
$$

Proof. Since by Lemma 3.17 the set of parabolic points is $G$-finite it is enough to prove the statement for a fixed parabolic point $p \in T$. By Lemma 3.18 the parabolic point $p$ is the unique limit point of $N_{d}(T(p))$. By definition of the topology on $T \sqcup$ Ent $T$ for sufficiently large $l_{0}$ our assumption implies that the entourages $\boldsymbol{c}$ and $\boldsymbol{d}$ are sufficiently close to $p$. By Lemma 6.3 the entourages $c^{*}$ and $\boldsymbol{d}^{*}$ are also close to $p$. So for every $\boldsymbol{b}^{*}$-small neighborhood $U_{p}$ of $p$ its complement $U_{p}^{\prime}$ is $c^{*}$-small and $\boldsymbol{d}^{*}$-small for $l>l_{0}$. Then $\operatorname{sh}_{\boldsymbol{b}^{*}} \boldsymbol{c}^{*} \subset U_{p}$ and $\operatorname{sh}_{\boldsymbol{b}^{*}} \boldsymbol{d}^{*} \subset U_{p}$. Therefore $\widetilde{\Delta}_{\boldsymbol{b}^{*}}\left(\operatorname{sh}_{\boldsymbol{b}^{*}} \boldsymbol{c}^{*}, \operatorname{sh}_{\boldsymbol{b}^{*}} \boldsymbol{d}^{*}\right) \leq 1$. We obtain

$$
\Delta_{\boldsymbol{b}^{*}}\left(\operatorname{sh}_{\boldsymbol{b}^{*}} \boldsymbol{d}^{*}, \operatorname{sh}_{\boldsymbol{b}^{*}} \boldsymbol{a}^{*}\right) \geq \Delta_{\boldsymbol{b}^{*}}\left(\operatorname{sh}_{\boldsymbol{b}^{*}} \boldsymbol{a}^{*}, \operatorname{sh}_{\boldsymbol{b}^{*}} \boldsymbol{c}^{*}\right)-\tilde{\Delta}_{\boldsymbol{b}^{*}}\left(\operatorname{sh}_{\boldsymbol{b}^{*}} \boldsymbol{c}^{*}, \operatorname{sh}_{\boldsymbol{b}^{*}} \boldsymbol{d}^{*}\right)>k-1 .
$$


Remark 6.6. (about the constants). Since now on we assume that the tightness constant $l$ is much bigger than the horosphericity constants $d, e=e(d)$ (see Definition 5.6 and the Remark after it) and $w$ (see Lemma 6.4). We will also suppose that the chosen constants satisfy the relations

$$
l_{0}>4 w \text { and } w>e .
$$

Proof of Theorem B. Recall that for a fixed constant $d>0$ by Lemma 6.2 we have found $\rho=\rho(d)$ and have defined the set $A^{*}$ of vertices of a new graph of entourages. Since now on the term "(non)-horosphericity" will mean " $d$-(non)horosphericity."

Before going into the details we outline the proof of the theorem. We start by choosing non-horospherical points $\boldsymbol{v}_{n}$ of the curve $\gamma$ which give by Lemma 6.4 an auxiliary tube $P^{*}=\left\{\boldsymbol{v}_{n}^{*}\right\}$ in the graph $A^{*}$. There is a quasi-geodesic $\alpha^{*} \subset A^{*}$ whose non-horospherical points are in a bounded distance from $P^{*}$ (Lemma 5.7). Since the graphs $\mathcal{G}$ and $\mathcal{G}^{*}$ are $G$-finite the map $\varphi: v \rightarrow v^{*}$ is a quasi-isometry between them. This will give us a quasi-geodesic $\alpha \subset A$ satisfying the statement of the Theorem. All the remaining constants will be found in the course of the proof.

To construct the tube $P^{*}$ we proceed inductively by choosing vertices of $\gamma$ as follows. Let $\gamma(0)$ be the first non-horospherical point on $\gamma$, then we put $v_{0}^{*}=$ $\gamma^{*}(0)$. Suppose that a point $v_{n}^{*}=\gamma^{*}(n)$ is already chosen. Then for the constant $w$ fixed above we choose $i_{n+1} \geq i_{n}+w$ such that $\gamma\left(i_{n+1}\right)$ is the first nonhorospherical point on $\gamma$ after $\gamma\left(i_{n}+w\right)$. We set $\boldsymbol{v}_{n+1}^{*}=\gamma^{*}\left(i_{n+1}\right)$. The following proposition shows that for every $n$ each three chosen neighboring vertices form a tube $v_{n-1}^{*}-v_{n}^{*}-v_{n+1}^{*}(k-2)$ for the integer $k$ fixed above. Then all the constructed vertices will give a tube $P^{*}=v_{0}^{*}-v_{1}^{*}-\cdots-v_{m}^{*}(k-2)$.

Proposition 6.7. For every $n \in \mathbb{N}$ one has $v_{n-1}^{*}-v_{n}^{*}-v_{n+1}^{*}(k-2)$.

Proof of Proposition 6.7. There are four different cases depending on the lengths

$$
|\gamma|\left[i_{n}, i_{n+1}\right] \mid=i_{n+1}-i_{n}
$$

of the parts of $\gamma(n \in \mathbb{N})$.

CASE 1. $i_{n}-i_{n-1} \leq l / 2 \wedge i_{n+1}-i_{n} \leq l / 2$. By definition of a tight curve the points $\gamma\left(i_{n-1}\right), \gamma\left(i_{n}\right), \gamma\left(i_{n+1}\right)$ belong to a $c$-quasigeodesic part of $\gamma$ so the result follows from Lemma 6.4. 
CASE $2 . i_{n+1}-i_{n-1}>l$. There are three subcases.

Su в CASE 2.1. $i_{n}-i_{n-1} \leq l / 2 \wedge i_{n+1}-i_{n}>l / 2$. Since $\gamma\left(i_{n+1}\right)$ is the first non-horospherical point on $\gamma$ after $\gamma\left(i_{n}+w\right)$ and $w<l / 2$ the point $\gamma\left(i_{n}+w\right)$ is horospherical. Since $w>e$ by the Remark after Definition 5.6 there exists a unique horosphere $T(p)$ such that $d_{A}\left(\gamma\left(i_{n}+w\right), T(p)\right) \leq d$. As $\left.\gamma\right|_{\left[i_{n}, i_{n}+w\right]}$ is a $c$-quasigeodesic we have

$$
d_{A}\left(\gamma\left(i_{n}\right), T(p)\right)<c w+c+d .
$$

Furthermore Lemma 6.4 yields

$$
\gamma^{*}\left(i_{n-1}\right)-\gamma^{*}\left(i_{n}\right)-\gamma^{*}\left(i_{n-1}+l\right)(k) .
$$

Since $i_{n+1}-i_{n-1}>l$ the point $\gamma\left(i_{n-1}+l\right)$ is also horospherical and $\gamma\left(i_{n-1}+l\right) \in$ $\left.\gamma\right|_{\left.] i_{n}+w, i_{n+1}\right]}$. The curve $\left.\gamma\right|_{\left[i_{n}, i_{n}+l\right]}$ is still $c$-quasigeodesic so we have

$$
d_{A}\left(\gamma\left(i_{n}\right), \gamma\left(i_{n-1}+l\right)\right)>\frac{i_{n-1}+l-i_{n}}{c}-c \geq \frac{l}{2 c}-c>\frac{l}{4 c},
$$

where we assume that $l>l_{0}>4 c^{2}$ for the constant $l_{0}$ from Lemma 6.5.

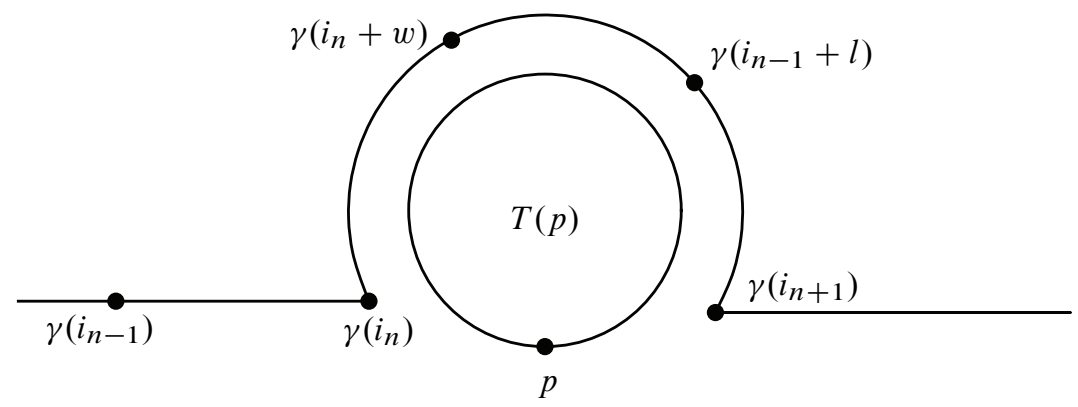

Figure 4. Tight curves around horospheres.

By construction we can also suppose that $\gamma\left(i_{n+1}\right) \in N_{d}(T(p))$ for the $d$ neighborhood $N_{d}(T(p))$ of $p$. Indeed otherwise there would exist another nonhorospherical point on $\gamma$ after $\gamma\left(i_{n}+w\right)$ and preceding $\gamma\left(i_{n+1}\right)$. So by $(* * *)$ $\left\{\gamma\left(i_{n}\right), \gamma\left(i_{n+1}\right)\right\} \subset N_{d_{0}}(T(p))$, where $d_{0}=c w+c+d$.

If, first, $i_{n+1}-i_{n} \leq l$ then $\left.\gamma\right|_{\left[i_{n}, i_{n+1}\right]}$ is a $c$-quasigeodesic, and

$$
d_{A}\left(\gamma\left(i_{n}\right), \gamma\left(i_{n+1}\right)\right)>\frac{l}{2 c}-c>\frac{l}{4 c} .
$$


Hence by the choice of $l_{0}$ (see Remark 6.6) and all $l>l_{0}$ we have from (2), (3), and Lemma 6.5

$$
\gamma^{*}\left(i_{n-1}\right)-\gamma^{*}\left(i_{n}\right)-\gamma^{*}\left(i_{n+1}\right)(k-1) .
$$

If now $i_{n+1}-i_{n}>l$ then applying Definition 6.1.2) to $N_{d_{0}}(p)$ we obtain

$$
d_{A}\left(\gamma\left(i_{n}\right), \gamma\left(i_{n+1}\right)\right)>c^{-1}(l)
$$

and again (4) follows from (2), (3), and Lemma 6.5.

Su BCASE 2.2. $i_{n}-i_{n-1} \geq l / 2 \wedge i_{n+1}-i_{n} \leq l / 2$. The argument is similar to that of Subcase 2.1 but it works in the opposite direction. We have the tube $\gamma^{*}\left(i_{n+1}\right)-$ $\gamma^{*}\left(i_{n}\right)-\gamma^{*}\left(i_{n+1}-l\right)(k)$. As above if $i_{n}-i_{n-1} \leq l$ then the curve $\left.\gamma\right|_{\left[i_{n-1}, i_{n}\right]}$ is $c$-quasigeodesic and so its diameter is greater than $l / 4 c$. If not then using the tightness property of it, we obtain that $d_{A}\left(\gamma\left(i_{n-1}\right), \gamma\left(i_{n}\right)\right)>c^{-1}(l)$ and (4) follows by the same argument as in Subcase 2.1.

SubCASE $2.3 . i_{n}-i_{n-1} \geq l / 2 \wedge i_{n+1}-i_{n} \geq l / 2$. In this case we have that the points $\gamma\left(i_{n}-l / 4\right)$ and $\gamma\left(i_{n}+l / 4\right)$ preceding respectively $\gamma\left(i_{n}\right)$ and $\gamma\left(i_{n+1}\right)$ are both horospherical. Indeed $w<l / 4$ and $\gamma\left(i_{n}\right)$ and $\gamma\left(i_{n+1}\right)$ are the first nonhorospherical points after $\gamma\left(i_{n-1}\right)$ and $\gamma\left(i_{n}\right)$ respectively. So we can suppose that $\gamma\left(i_{n}\right) \in N_{d}(T(p))$ and $\gamma\left(i_{n+1}\right) \in N_{d}(T(q))$ where $p$ and $q$ are distinct parabolic points. Since $\left.\gamma\right|_{\left[i_{n}-l / 4, i_{n}+l / 4\right]}$ is a quasigeodesic by Lemma 6.4 we have

$$
\gamma^{*}\left(i_{n}-\frac{l}{4}\right)-\gamma^{*}\left(i_{n}\right)-\gamma^{*}\left(i_{n}+\frac{l}{4}\right)(k)
$$

We also have $d_{A}\left(\gamma\left(i_{n-1}\right), \gamma\left(i_{n}\right)\right)$ and $d_{A}\left(\gamma\left(i_{n}\right), \gamma\left(i_{n+1}\right)\right)$ are both greater than $l / 4 c$. Indeed if $i_{n}-i_{n-1}>l$ then by $(l, c)$-tightness we have $d_{A}\left(\gamma\left(i_{n-1}\right), \gamma\left(i_{n}\right)\right)>$ $c^{-l}(l)>l / 4 c$. If $i_{n}-i_{n-1} \leq l$ then $\left.\gamma\right|_{\left[i_{n-1}, i_{n}\right]}$ is $c$-quasigeodesic, and as above $d_{A}\left(\gamma\left(i_{n-1}\right), \gamma\left(i_{n}\right)\right)>l / 4 c$. In the same way we obtain $d_{A}\left(\gamma\left(i_{n}\right), \gamma\left(i_{n+1}\right)\right)>l / 4 c$.

Applying now Lemma 6.5 to (5) two times for $l>4 c l_{0}$ we obtain

$$
\gamma^{*}\left(i_{n-1}\right)-\gamma^{*}\left(i_{n}\right)-\gamma^{*}\left(i_{n+1}\right)(k-2)
$$

The proposition is proved.

We continue the proof of Theorem B. By Proposition 6.7 the curve $\gamma$ admits a set of non-horospherical points $\boldsymbol{v}_{n}=\gamma\left(i_{n}\right)$ such that $\boldsymbol{v}_{n}^{*}=\varphi\left(\gamma\left(i_{n}\right)\right)$ is a vertex of the tube $P^{*}$. Let $\boldsymbol{u}=\gamma(i)$ be a non-horospherical point of $\gamma$ which does not 
belong to the set $\left\{\boldsymbol{v}_{n}\right\}_{n}$. Then by construction $i_{n} \leq i<i_{n}+w$ for some $i_{n} \in$ $\{0, \ldots, m\}$. Since $w<l$ the curve $\left.\gamma\right|_{\left[i_{n}, i_{n}+w\right]}$ is a $c$-quasigeodesic so $d_{A}\left(\boldsymbol{v}_{n}, \boldsymbol{u}\right) \leq$ $c w+c$. The map $\varphi: \boldsymbol{u} \in A \rightarrow \boldsymbol{u}^{*} \in A^{*}$ is a quasi-isometry so $d_{A^{*}}\left(\boldsymbol{u}^{*}, \boldsymbol{v}_{n}^{*}\right) \leq$ $w_{1}$ for some uniform constant $w_{1}>0$. Let $\alpha^{*}$ be a geodesic in the graph $\mathcal{G}^{*}$ with the same endpoints as $P^{*}$. Then by Lemma 5.2 (applied to the graph $\mathcal{G}^{*}$ ) there is a constant $D^{*}>0$ such that, for all $\boldsymbol{v}^{*} \in P^{*}, d_{A^{*}}\left(\alpha^{*}, \boldsymbol{v}^{*}\right) \leq D^{*}$. So for every non-horospherical point $\boldsymbol{u} \in \gamma$ we have $d_{A^{*}}\left(\boldsymbol{u}^{*}, \alpha^{*}\right) \leq d_{A^{*}}\left(\boldsymbol{u}^{*}, \boldsymbol{v}^{*}\right)+$ $d_{A^{*}}\left(\boldsymbol{v}^{*}, \alpha^{*}\right) \leq w_{1}+D^{*}$ where $\boldsymbol{v}^{*} \in P^{*}$. The map $\varphi^{-1}: \boldsymbol{u}^{*} \rightarrow \boldsymbol{u}$ is a quasiisometry too. Hence $\alpha=\varphi^{-1}\left(\alpha^{*}\right)$ is a $c_{0}$-quasi-geodesic in $\mathcal{G}$ such that for every non-horospherical point $\boldsymbol{u} \in \gamma$ we have $d_{A}(\boldsymbol{u}, \alpha) \leq w_{0}$ for some positive constants $c_{0}$ and $w_{0}$. Theorem $\mathrm{B}$ is proved.

\section{Floyd quasiconvexity of parabolic subgroups}

Let $G \curvearrowright T$ be a 3-discontinuous and 2-cocompact action of a finitely generated group $G$ on a compactum $T$. Let $\Gamma$ be a locally finite, connected graph on which $G$ acts discontinuously and cofinitely (e.g. its Cayley graph or the graph of entourages). We denote by $d\left(\right.$, ) the graph distance of $\Gamma$. Let $f: \mathbb{N} \rightarrow \mathbb{R}_{>0}$ be a scaling function esatisfying the following conditions (see Section 4): there exists $\lambda>0$ such that, for all $n \in \mathbb{N}$,

$$
\begin{gathered}
1<\frac{f(n)}{f(n+1)}<\lambda ; \\
\sum_{n \in \mathbb{N}} f(n)<+\infty .
\end{gathered}
$$

To precise that $f$ satisfies (1) with respect to some $\lambda \in] 1, \infty[$ we will say that the function $f$ is $\lambda$-slow. Denote by $\delta_{f}$ the corresponding Floyd metric on $\Gamma$ with respect to a fixed vertex $v \in \Gamma^{0}$.

By a standard argument based on Arzela-Ascoli theorem it follows that the Floyd completion $\bar{\Gamma}_{f}$ of the graph $\Gamma$ is a geodesic (strictly intrinsic) space; see e.g. [8, Theorem 2.5.14]. We call Floyd geodesic (or $\delta_{f}$-geodesic) a geodesic in the space $\bar{\Gamma}_{f}$ with respect to the Floyd $\delta_{f}$-metric. The geodesics in $\Gamma$ with respect to the graph distance $d$ we call below ( $d$-)geodesics.

The set $\Gamma^{0} / G=K$ is finite so we can identify in $\Gamma$ a subgroup $H$ of $G$ with the orbit $H K=\bigcup_{h \in H} h K \subset \Gamma^{0}$. Let $N_{R}(H)$ denote the $R$-neighborhood of $H K$ in $\Gamma$ for the graph metric. 
Definition 7.1. Let $\Gamma$ be a locally finite, connected graph possessing a $G$-finite action. A subgroup $H$ of $G$ is called Floyd quasiconvex in $\Gamma$ if there exists a constant $R=R(H)>0$ such that every Floyd geodesic $\gamma=\gamma\left(h_{1}, h_{2}\right) \subset \Gamma$ for the metric $\delta_{f}$ having the endpoints $h_{i}$ in $H$ belongs to $N_{R}(H)$ for all $x \in$ $\gamma: d(x, H)<R$.

By Corollary 5.5 every parabolic subgroup of $G$ is quasiconvex with respect to the word metric (see also [15]). The aim of this section is to prove the following Theorem stating the Floyd quasiconvexity of parabolic subgroups.

Theorem C. Let $G$ be a finitely generated group acting 3-discontinuously and 2-cocompactly on a compactum $T$. Let $\Gamma$ be a locally finite, connected graph admitting a cocompact discontinuous action of $G$. Then there exists a constant $\left.\lambda_{0} \in\right] 1, \infty[$ such that for every $\lambda \in] 1, \lambda_{0}[$ and every $\lambda$-slow Floyd scaling function $f$ satisfying (1) and (2), each parabolic subgroup $H$ of $G$ is Floyd quasiconvex for the Floyd metric $\delta_{f}$.

We start with two lemmas.

Lemma 7.2. For every $r>0$ there exists $\lambda_{0}>1$ such that for all $\left.\lambda \in\right] 1, \lambda_{0}[$ and every $\lambda$-slow function $f$ the condition $d(x, y) \leq r\left(x, y \in \Gamma^{0}\right)$ implies that every Floyd $\delta_{f}$-geodesic $\gamma=\gamma(x, y) \subset \Gamma$ whose endpoints are $x$ and $y$ is a geodesic in $\Gamma$.

Remark. A similar statement for $\delta$-hyperbolic spaces is proved in [20, Lemma 7.2.1].

Proof. Let $v \in \Gamma^{0}$ be a basepoint. Denote by $\omega=\omega(x, y)$ a $\Gamma$-geodesic between $x$ and $y$ for which $|\omega|=r$. Let $m \in \omega$ such that $d(v, m)=d(v, \omega)$. Then for at least one of the points $x$ or $y$, say $x$, we have $d(v, x) \geq d(m, x)$. Indeed otherwise $d(x, y) \leq d(v, x)+d(y, v)<r$ which is impossible. Put $R=d(v, x)$. We have

$$
L_{f}(\omega)=\sum_{i=1}^{r} f\left(d\left(v,\left\{x_{i}, x_{i+1}\right\}\right)\right) \leq r f(d(v, m)) .
$$

Suppose by contradiction that $\gamma$ is not $d$-geodesic and so $|\gamma| \geq r+1$. Let $\gamma^{\prime}$ be the part of $\gamma$ in the ball $B(v, R+r+1)$ of radius $R+r+1$ centered 
at $v$. By the triangle inequality we also have $\left|\gamma^{\prime}\right| \geq r+1$. So $L_{f}(\gamma) \geq L_{f}\left(\gamma^{\prime}\right) \geq$ $(r+1) f(R+r+1)$. We obtain

$$
\begin{aligned}
\frac{f(R+r+1)}{f(R-d(x, m))} & \leq \frac{f(R+r+1)}{f(d(v, m))} \\
& \leq \frac{L_{f}(\gamma)}{(r+1) f(d(v, m))} \\
& \leq \frac{L_{f}(\omega)}{(r+1) f(d(v, m))} \\
& \leq \frac{r}{r+1} .
\end{aligned}
$$

Since $f$ is $\lambda$-slow we have

$$
\frac{f(r+R+1)}{f(R-d(x, m))}>\frac{1}{\lambda^{r+d(m, x)+1}}>\frac{1}{\lambda^{2 r+1}} .
$$

Thus

$$
\frac{1}{\lambda^{2 r+1}}<\frac{r}{r+1} \text {. }
$$

Then there exists $\lambda_{0}>1$ such that for $\left.\lambda \in\right] 1, \lambda_{0}[$ inequality $(*)$ is not true for a fixed $r>0$. So for such $\lambda_{0}$ we have a contradiction. The lemma is proved.

Remark. Obviously if $r$ is not fixed and tends to infinity the above constant $\lambda_{0}$ does not exist.

The group $G$ acts discontinuously and cofinitely on the graph $\Gamma$ and on the graph $\mathcal{G}$ of entourages (see Section 3). Since the set $\Gamma^{0} / G=K$ is finite and $\mathcal{G}^{0} / G=\left\{\boldsymbol{a}_{0}\right\}\left(\boldsymbol{a}_{0} \in A\right)$ the correspondence $K \rightarrow \boldsymbol{a}_{0}$ extends $G$-equivariantly to the quasi-isometry $\psi: g K \rightarrow g \boldsymbol{a}_{0}(g \in G)$. In the same way we define the inverse quasi-isometric map $\psi^{-1}: \mathcal{G} \rightarrow \Gamma$ for which $\psi^{-1}\left(\boldsymbol{a}_{0}\right) \in K$.

For a parabolic point $p \in \mathcal{P}$ let $H$ denote the stabilizer of $p$ in $G$.

Lemma 7.3. The map $\psi$ extends continuously by the identity map to the map $\Gamma \sqcup \mathcal{P} \rightarrow \mathcal{G} \sqcup \mathcal{P}$. Furthermore for any $d>0$ there exists $d^{\prime}=d^{\prime}(d, p)$ such that $\psi\left(N_{d}(H)\right)$ belongs to a d'-neighborhood $N_{d^{\prime}}(T(p))$ of the horosphere $T(p) \subset \mathcal{G}$, and conversely $\psi^{-1}\left(N_{d}(T(p)) \subset N_{d^{\prime}}(H)\right.$.

Proof. It follows from [17, Lemma 3.8] that the unique limit point of $N_{d}(H)$ on $T$ is $p$. The set $\psi\left(N_{d}(H)\right)$ is an $H$-finite subset of $\mathcal{G}$ and so belong to $N_{d^{\prime}}\left(T_{p}\right)$ for some $d^{\prime}=d^{\prime}(d, p)$ (see also the proof of Corollary 5.5). Since the unique limit point of the set $N_{d^{\prime}}\left(T_{p}\right)$ is also $p$ the map $\psi$ extends identically to the set $\mathcal{P}$. The second statement is similar. 
Lemma 7.4. For every $l>0$ and $\varepsilon>0$ there exists $\lambda_{0}>1$ such that for any $\lambda \in] 1, \lambda_{0}\left[\right.$ and $\lambda$-slow function $f$ satisfying (1) and (2) one has: if $\gamma \subset \Gamma$ is $\delta_{f}$ geodesic then the curve $\psi(\gamma) \subset \mathcal{G}$ is $(l, c)$-tight where $c$ is the quasi-isometry constant of $\psi$.

Proof. For a fixed $l>0$ by Lemma 7.2 (applied to $r=l$ ) there exists $\lambda_{0}>1$ such that for any $\lambda \in] 1, \lambda_{0}$ [ and any $\lambda$-slow function $f$, every part of $\gamma$ of length less than $l$ is geodesic in $\Gamma$. Then $\beta=\psi(\gamma)$ is $c$-quasigeodesic on every interval of length at most $l$. So the first condition of Definition 6.1 is satisfied for $\beta \subset \mathcal{G}$.

To verify condition 2) of Definition 6.1, assume that

$$
|\beta(J)|>l,
$$

If first $\operatorname{diam}(\partial \gamma(J)) \leq l$ then again by Lemma $\left.7.2 \gamma\right|_{J}$ is geodesic in $\Gamma$. So $\left.\beta\right|_{J}$ is $c$-quasigeodesic in $\mathcal{G}$. It follows from (**) that

$$
\operatorname{diam}(\partial(\beta(J)))>c^{-1}(l)=\frac{l}{c}-c
$$

If now $\operatorname{diam}(\partial \gamma(J))>l$ then we have $\mid \partial \beta(J)) \mid>c^{-1}(l)$ since $\psi$ is a $c$-quasiisometry. The lemma is proved.

Note that the proof of Lemma 7.4 does not use the horospheres to prove the tightness condition of Definition 6.1.2). The needed property holds for any part of $\beta$ of length bigger than $l$. The following Corollary shows that it remains valid for a curve in $\Gamma$ close in the Floyd metric to a Floyd geodesic if the latter one does not belong to the graph.

Corollary 7.5. For every $l>0$ there exists $\lambda_{0}>1$ such that for every $\left.\lambda \in\right] 1, \lambda_{0}[$ and $\lambda$-slow function $f$ if the Floyd geodesic $\gamma[x, y] \subset \Gamma_{f}$ joining two distinct points $x$ and $y$ does not belong to $\Gamma$, then there exists a curve $\tilde{\gamma}[x, y] \subset \Gamma$ between $x$ and $y$ such that $\left|L_{f}(\tilde{\gamma})-L_{f}(\gamma)\right| \leq \varepsilon$ and every part of $\tilde{\gamma}$ of length $l$ is $d$-geodesic.

Furthermore the curve $\psi(\tilde{\gamma}) \subset \mathcal{G}$ is $(l, c)$-tight for the quasi-isometry constant $c$.

Proof. For a fixed $l$ we choose $\lambda$-slow function $f$ such that $\lambda \geq l /(l+1)$. Suppose that a Floyd geodesic $\gamma[x, y]$ intersects the Floyd boundary $\partial_{f} \Gamma$. Then for any $\varepsilon>0$ there exists a curve

$$
\hat{\gamma}: I \longrightarrow \Gamma
$$

such that

$$
\hat{\gamma}(\partial I)=\{x, y\}
$$


and

$$
\left|L_{f}(\hat{\gamma})-L_{f}(\gamma)\right|<\varepsilon \text {. }
$$

Let $x^{\prime}$ and $y^{\prime}$ be two points on $\hat{\gamma}$ such that $d\left(x^{\prime}, y^{\prime}\right)=l$. If the part $\hat{\gamma}\left[x^{\prime}, y^{\prime}\right]$ of $\hat{\gamma}$ between them is not $d$-geodesic we replace it by a $d$-geodesic $\omega=\omega\left[x^{\prime}, y^{\prime}\right]$ between $x^{\prime}$ and $y^{\prime}$. Then the $d$-length of the obtained curve $\tilde{\gamma}$ is strictly less than that of $\hat{\gamma}$. Furthermore by Lemma 7.2 (applied to $r=l$ ) the curve $\omega$ is also a Floyd geodesic. So we have

$$
L_{f}(\gamma) \leq L_{f}(\tilde{\gamma}) \leq L_{f}(\hat{\gamma}) \leq L_{f}(\gamma)+\varepsilon
$$

Repeating this procedure with every pair of points of $\tilde{\gamma}$ situated at the distance $l$ we strictly decrease its $d$-length. Since $d(x, y) \in \mathbb{Z}_{>0}$ after finitely many steps we obtain a curve (still denoted by $\tilde{\gamma}$ ) satisfying the first statement.

Since $\psi: \Gamma \rightarrow \mathcal{G}$ is a $c$-quasi-isometry the last part follows from the argument of Lemma 7.4.

Proof of Theorem $\mathrm{C}$. The group $G$ acts 3-discontinuously and 2-cocompactly on a compactum $T$. Let $\Gamma$ be a locally finite, connected graph admitting cocompact discontinuous action of $G$.

Let $l_{0}$ and $\lambda_{0}$ be the constants given by Theorem B and Lemma 7.4 (or Corollary 7.5). Let $f$ be a $\lambda$-slow function for $\lambda \in] 1, \lambda_{0}\left[\right.$. Suppose that $\gamma=\gamma\left(h_{1}, h_{2}\right) \subset$ $\Gamma$ is a $\delta_{f}$-geodesic between two elements $h_{1}$ and $h_{2}$ in the parabolic subgroup $H$. Then by Lemma 7.4 the curve $\beta=\psi(\gamma)$ is $(l, c)$-tight in $\mathcal{G}$.

A segment of a curve $\beta \subset \mathcal{G}$ having the extremities at points $\boldsymbol{h}_{i} \in \mathcal{G}(i=1,2)$ we denote by $\beta\left[\boldsymbol{h}_{1}, \boldsymbol{h}_{2}\right]$. By Lemma 7.3 for every $d>0$ and $p \in \mathcal{P}$ there exists $d^{\prime}=d^{\prime}(d, p)$ such that the set $\psi^{-1}\left(N_{d}\left(T_{p}\right)\right)$ belongs to $N_{d^{\prime}}(H)$. So Theorem C follows from the following.

Proposition 7.6. For every $c>0$ there exist positive constants $s, d$ and $l_{0}$ such that for all $l>l_{0}$ every $(l, c)$-tight curve $\beta\left[\boldsymbol{h}_{1}, \boldsymbol{h}_{2}\right] \subset \mathcal{G}$ with $\boldsymbol{h}_{i} \in N_{d}(T(p))$ $(i=1,2)$ is situated in $N_{s}(T(p))$ for some $p \in \mathcal{P}$.

Proof of the proposition. Since $\mathcal{P}$ is $G$-finite it is enough to prove the statement for a fixed $p \in \mathcal{P}$. Suppose that $\beta$ is a $(l, c)$-tight curve where $l>l_{0}$ and the constants $l_{0}$ and $c$ are given by Theorem $\mathrm{B}$. So there exists a $c^{\prime}$-quasigeodesic $\alpha \subset \mathcal{G}$ such that every non-horospherical point $v$ of $\beta$ belongs to the $w_{0}$-neighborhood $N_{w_{0}}(\alpha)$ with respect to the distance $d_{A}$. By Lemma 5.4.1), for all $i \in I$

$$
d_{A}(\alpha(i), T(p)) \leq \text { const. }
$$


Thus there exists a constant $R=R(d)>0$ such that for any non-horospherical point $v \in \beta$ we have $d_{A}(v, T(p)) \leq R$.

Let now $\beta[\boldsymbol{x}, \boldsymbol{y}]$ be a $d$-horospherical part of $\beta$ lying in $N_{d}(T(q))$ of another parabolic point $q$. Up to increasing the above part of $\beta$ we can suppose that both extremal points $\boldsymbol{x}$ and $\boldsymbol{y}$ are non-horospherical. So we have $d_{A}(\boldsymbol{x}, T(p)) \leq R$ and $d_{A}(\boldsymbol{y}, T(p)) \leq R$. Let $\boldsymbol{x}_{1}$ and $\boldsymbol{y}_{1}$ be points on $T(p)$ realizing these distances respectively. Denote by $\alpha_{1}=\left[\boldsymbol{x}, \boldsymbol{x}_{1}\right]$ and $\alpha_{2}=\left[\boldsymbol{y}, \boldsymbol{y}_{1}\right]$ the corresponding geodesics (see the figure below).

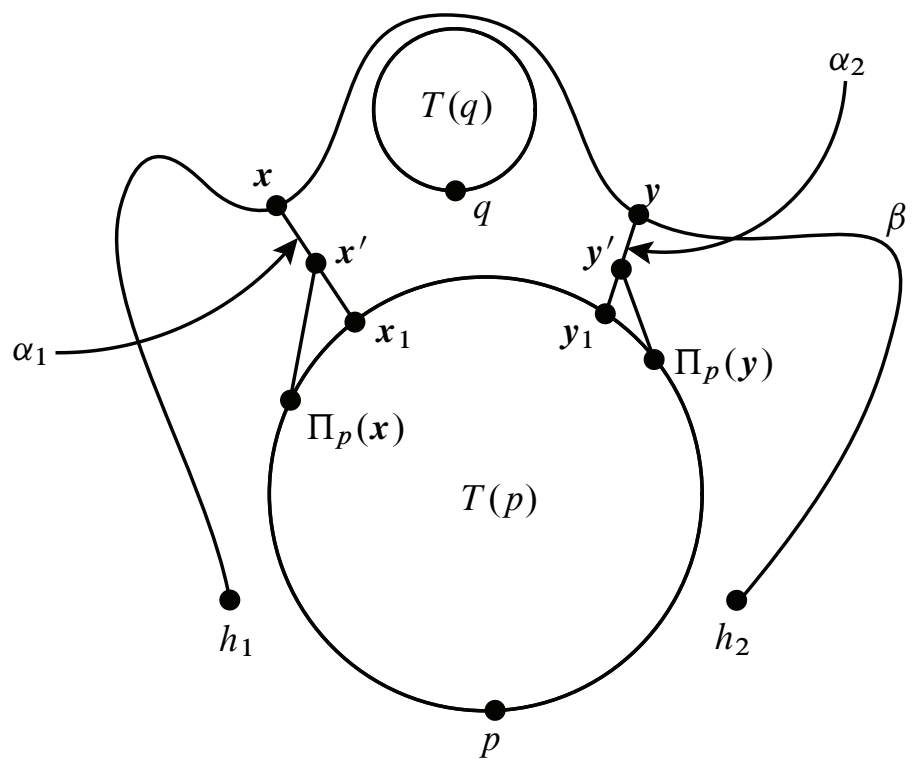

Let $\Pi_{p}(\boldsymbol{x})$ and $\Pi_{p}(\boldsymbol{y})$ be the projections of $\boldsymbol{x}$ and $\boldsymbol{y}$ on $T(p)$. By Lemma 5.3.1) we have $d_{A}\left(\alpha_{1}, \Pi_{p}(\boldsymbol{x})\right)=d_{A}\left(\boldsymbol{x}^{\prime}, \Pi_{p}(\boldsymbol{x})\right) \leq L$ for some constant $L$ depending only on $R$, where $\boldsymbol{x}^{\prime} \in \alpha_{1}$. Hence $d_{A}\left(\boldsymbol{x}, \Pi_{p}(\boldsymbol{x})\right) \leq R+L$ and similarly $d_{A}\left(y, \Pi_{p}(y)\right) \leq R+L$. By Proposition 3.32.2) the set $\Pi_{p}(T(q))$ is finite and so is $\Pi_{p}\left(N_{d}(T(q))\right)$. So there exists a constant $C>0$ such that $d_{A}\left(\Pi_{p} x, \Pi_{p} y\right) \leq$ $C$. Therefore $d_{A}(\boldsymbol{x}, \boldsymbol{y}) \leq C+2 R+2 L$. The above constants $C, R$ and $L$ depend only on $p$ so we can choose the parameter $l$ from Theorem B satisfying $l>\max \left(l_{0}, C+2 R+2 L\right)$. Then the segment $\beta[\boldsymbol{x}, \boldsymbol{y}]$ is $c$-quasigeodesic whose length is bounded by $c(C+2 R+2 L)+c$. Hence $\beta[x, y] \subset N_{s}(T(p))$ where $s=R+c(C+2 R+2 L)+c$. Theorem $C$ is proved.

Since every parabolic subgroup $H$ is quasiconvex in $G$ there exists a quasiisometric map $\varphi$ of the group $H$ into the graph $\Gamma$. We have the following. 
Corollary 7.7. For the constant $\lambda_{0}$ from Theorem $\mathrm{C}$ and every $\left.\lambda \in\right] 1, \lambda_{0}[$ let $f$ be a $\lambda$-slow Floyd function satisfying in addition the following assumption:

$$
\frac{f(n)}{f(2 n)} \leq \kappa, \quad n \in \mathbb{N},
$$

for some constant $\kappa>0$. Let $p$ be a parabolic point for the action of $G$ on $T$ and $H=\operatorname{Stab}_{G} p$ be its stabilizer. Then $\varphi$ extends injectively to the Floyd boundaries:

$$
\varphi: \bar{H}_{f} \longrightarrow \bar{\Gamma}_{f}
$$

Remark. Note that every polynomial type function $f(n)=(n+1)^{-k}(k>1)$ satisfies conditions (1)-(3) for any fixed $\lambda>1$ and $\kappa>0\left(n>n_{0}\right)$.

Proof of Corollary 7.7. We suppose that $H \subset \Gamma^{0}$ and $\varphi: H \hookrightarrow \Gamma^{0}$ is the identity map inducing the quasi-isometry between the word metrics. Let $d^{\prime}(\cdot, \cdot)$ and $d(\cdot, \cdot)$ be the graph distances of $H$ and $\Gamma$ respectively. We also denote by $\delta_{f, H}$ and $\delta_{f, G}$ the corresponding Floyd distances with respect to a fixed basepoint $v \in H$. Since $f$ satisfies (3) by [17, Lemma 2.5] the map $\varphi$ extends to a Lipschitz map (denoted by the same letter) $\varphi: \bar{H}_{f} \rightarrow \bar{\Gamma}_{f}$ between the Floyd completions of $H$ and $\Gamma$.

Let $x, y \in H \subset \Gamma$ be two distinct points. If the Floyd geodesic between $x$ and $y$ belongs to $\Gamma$ we denote it by $\gamma$; if not for any $\varepsilon \in] 0,1[$ let $\gamma$ be the $(l, c)$-tight curve $\left(l>l_{0}\right)$ given by Corollary 7.5 whose Floyd length is $\varepsilon$-close to that of the Floyd geodesic. In the first case by Theorem C there exists a constant $R=R(H)$ such that that $\gamma \subset N_{R}(H)$, and in the second case the same conclusion for the curve $\gamma$ follows from Proposition 7.6.

We have $L_{f}(\gamma)=\sum_{i=1}^{l} f\left(d\left(v,\left\{x_{i}, x_{i+1}\right\}\right)\right)$. Denote by $x_{i}^{\prime} \in H$ one of the closest vertices to $x_{i}$ in $H(i=1, \ldots, l)$. By Theorem $\mathrm{C}$ there exists a constant $R>0$ such that $d\left(x_{i}, x_{i}^{\prime}\right) \leq R$. Thus $d\left(x_{i}^{\prime}, x_{i+1}^{\prime}\right) \leq 2 R+1$. So for any vertex $x_{i j}^{\prime}$ on a geodesic in $H$ between $x_{i}^{\prime}$ and $x_{i+1}^{\prime}$ we obtain $d\left(v,\left\{x_{i}, x_{i+1}\right\}\right) \leq$ $(3 R+1)+d\left(v, x_{i j}^{\prime}\right)$. Since $\varphi$ is quasi-isometric we have $1 / \alpha \cdot d^{\prime}\left(v, x_{i j}^{\prime}\right)-\beta \leq$ $d\left(v, x_{i j}^{\prime}\right) \leq \alpha d^{\prime}\left(v, x_{i j}^{\prime}\right)+\beta$ for some constants $\alpha$ and $\beta$. Let $\gamma^{\prime}=\gamma^{\prime}(x, y) \subset H$ be the curve between $x$ and $y$ obtained by connecting the vertices $x_{i}^{\prime}$ and $x_{i+1}^{\prime}$ by geodesics segments in $H$ passing through $x_{i j}^{\prime}$. We have $\alpha \cdot\left(d\left(x_{i}^{\prime}, x_{i+1}^{\prime}\right)+\beta\right)$. $f\left(d\left(v,\left\{x_{i}, x_{i+1}\right\}\right)\right) \geq d^{\prime}\left(x_{i}^{\prime}, x_{i+1}^{\prime}\right) \cdot f\left(d\left(v,\left\{x_{i}, x_{i+1}\right\}\right)\right)=\sum_{j} f\left(d\left(v,\left\{x_{i}, x_{i+1}\right\}\right)\right.$. Thus

$$
f\left(d\left(v,\left\{x_{i}, x_{i+1}\right\}\right)\right) \geq \frac{1}{2 \alpha R+\beta+\alpha} \sum_{j} f\left(\alpha d^{\prime}\left(v, x_{i j}^{\prime}\right)+m_{1}\right),
$$


where $m_{1}=\beta+3 R+1$. Conditions (1) and (3) yield

$$
L_{f, G}(\gamma) \geq \frac{L_{f, H}\left(\gamma^{\prime}\right)}{(2 \alpha R+\beta+\alpha) \lambda^{m_{1}} \kappa^{k_{1}}} \geq \frac{\delta_{f, H}(x, y)}{(2 \alpha R+\beta+\alpha) \lambda^{m_{1}} \kappa^{k_{1}}},
$$

where $k_{1}=\min \left\{k: 2^{k}>\alpha\right\}$. Since for every $\left.\varepsilon \in\right] 0,1[$ there exists a curve $\gamma$ satisfying (5) and for which $L_{f, G}(\gamma) \leq \delta_{f, G}(x, y)+\varepsilon$ we have

$$
\delta_{f, G}(x, y) \geq \frac{1}{(2 \alpha R+\beta+\alpha) \lambda^{m_{1}} \kappa^{k_{1}}} \cdot \delta_{f, H}(x, y) \quad \text { for all } x, y \in H .
$$

By continuity, inequality (6) remains valid for every pair of distinct points $x, y \in \bar{H}_{f}$. So the map $\varphi: \bar{H}_{f} \rightarrow \bar{\Gamma}_{f}$ is injective. The corollary is proved.

If $G$ acts on $T$ is 3-discontinuously and 2-cocompactly then the kernel of the equivariant Floyd map $F$ from the Floyd boundary $\partial_{f} G$ of the Cayley graph of $G$ to $T$ is described in [17, Theorem A]. Namely if it is not a single point then it is equal to the topological boundary $\partial\left(\operatorname{Stab}_{G} p\right)$ of the stabilizer $\operatorname{Stab}_{G} p$ of a parabolic point $p \in T$. We denote by $\partial_{f} \operatorname{Stab}_{G} p$ the Floyd boundary of $\operatorname{Stab}_{G} p$ corresponding to the function $f$. By Corollary 7.7 we have that $\left.\varphi\right|_{\partial_{f} H}$ is a homeomorphism. So the following is immediate.

Corollary 7.8. For every $\lambda \in] 1, \lambda_{0}[$ and each $\lambda$-slow function $f$ satisfying (1)-(3) one has

$$
F^{-1}(p)=\partial_{f}\left(\operatorname{Stab}_{G} p\right),
$$

for every parabolic point $p \in T$.

Corollary 7.8 answers positively our question $[17,1.1]$ and provides complete generalization of the theorem of Floyd [12] for the relatively hyperbolic groups.

\section{References}

[1] A. Beardon and B. Maskit, Limit points of Kleinian groups and finite sided fundamental polyhedra. Acta Math. 132 (1974), 1-12. Zbl 0277.30017 MR 0333164

[2] B. H. Bowditch, Relatively hyperbolic groups. Internat. J. Algebra Comput. 22 (2012), no. 3, article id. 1250016, 66 pp. Zbl 1259.20052 MR 2922380

[3] B. H. Bowditch, Convergence groups and configuration spaces. In J. Cossey, Ch. F. Miller III, W. D. Neumann, and M. Shapiro (eds.), Geometric group theory down under. (Canberra, July 14-19, 1996.) Walter de Gruyter \& Co., Berlin, 1999, 23-54. Zbl 0952.20032 Zbl 0910.00040 (collection) MR 1714838 MR 1714835 (collection) 
Non-finitely generated relatively hyperbolic groups and Floyd quasiconvexity

[4] B. H. Bowditch, A topological characterisation of hyperbolic groups. J. Amer. Math. Soc. 11 (1998), no. 3, 643-667. Zbl 0906.20022 MR 1602069

[5] B. H. Bowditch, A short proof that a subquadratic isoperimetric inequality implies a linear one. Michigan Math. J. 42 (1995), no. 1, 103-107. Zbl 0835.53051 MR 1322192

[6] M. Bridson and A. Haefliger, Metric spaces of non-positive curvature. Grundlehren der Mathematischen Wissenschaften, 319. Springer, Berlin etc., 1999. Zbl 0988.53001 MR 1744486

[7] N. Bourbaki, Éléments de mathématique. Topologie générale. Chapitres 1 à 4. Hermann, Paris, 1971. Zbl 0249.54001 MR 0358652

[8] D. Burago, Y. Burago, and S. Ivanov, A course in metric geometry. Graduate Studies in Mathematics, 33. American Mathematical Society, Providence, R.I., 2001. Zbl 0981.51016 MR

[9] F. Dahmani, Les groupes relativement hyperboliques et leurs bords. Ph.D. Thesis. Université Louis Pasteur (Strasbourg I), Strasbourg, 2003. http://cds.cern.ch/record/851759/files/cer-\%20002534906.ps.gz

[10] C. Drutu and M. Sapir, Tree-graded spaces and asymptotic cones of groups. With an appendix by D. Osin and M. Sapir. Topology 44 (2005), no. 5, 959-1058. Zbl 1101.20025 MR 2153979

[11] B. Farb, Relatively hyperbolic groups. Geom. Funct. Anal. 8 (1998), no. 5, 810-840. Zbl 0985.20027 MR 1650094

[12] W. J. Floyd, Group completions and limit sets of Kleinian groups. Invent. Math. 57 (1980), no. 3, 205-218. Zbl 0428.20022 MR 0568933

[13] E. M. Freden, Properties of convergence groups and spaces. Conform. Geom. Dyn. 1 (1997), 13-23 (electronic). Zbl 1452414 MR 1452414

[14] H. Furstenberg, Poisson boundaries and envelopes of discrete groups. Bull. Amer. Math. Soc. 73 (1967), 350-356. Zbl 0184.33105 MR 0210812

[15] V. Gerasimov, Expansive convergence groups are relatively hyperbolic. Geom. Funct. Anal. 19 (2009), no. 1, 137-169. Zbl 1226.20037 MR 2507221

[16] V. Gerasimov, Floyd maps for relatively hyperbolic groups. Geom. Funct. Anal. 22 (2012), no. 5, 1361-1399. Zbl 1276.20050 MR 2989436

[17] V. Gerasimov and L. Potyagailo, Quasi-isometric maps and Floyd boundaries of relatively hyperbolic groups. J. Eur. Math. Soc. (JEMS) 15 (2013), no. 6, 2115-2137. Zbl 1292.20047 MR 3120738

[18] V. Gerasimov and L. Potyagailo, Quasiconvexity in the relatively hyperbolic groups. Preprint 2011. arXiv:1103.1211 [math.GR]

[19] F. W. Gehring and G. J. Martin, Discrete quasiconformal groups I. Proc. London Math. Soc. (3) 55 (1987), no. 2, 331-358. Zbl 0628.30027 MR 0896224

[20] M. Gromov, Hyperbolic groups. In S. M. Gersten (ed.), Essays in group theory. Springer, New York, 1987, 75-263. Zbl 0634.20015 Zbl 0626.00014 (collection) MR 0919829 MR 0919826 (collection) 
[21] M. Gromov, Asymptotic invariants of infinite groups. Geometric group theory. Vol. 2. (Sussex University symposium, Brighton, July 14-19, 1991) Cambridge University Press, Cambridge, 1993. Zbl 0841.20039 MR 1253544

[22] D. Groves and J. F. Manning, Dehn filling in relatively hyperbolic groups. Israel J. Math. 168 (2008), 317-429. Zbl 1211.20038 MR 2448064

[23] C. Hruska, Relative hyperbolicity and relative quasiconvexity for countable groups. Algebr. Geom. Topol. 10 (2010), no. 3, 1807-1856. Zbl 1202.20046 MR 2684983

[24] A. Karlsson, Free subgroups of groups with non-trivial Floyd boundary. Comm. Algebra 31 (2003), no. 11, 5361-5376. Zbl 1036.20032 MR 2005231

[25] P. J. Myrberg, Untersuchungen ueber die automorphen Funktionen beliebiger vieler Variabelen. Acta Math. 46 (1925), 215-336.

[26] D. Osin, Relatively hyperbolic groups: intrinsic geometry, algebraic properties, and algorithmic problems. Mem. Amer. Math. Soc. 179 (2006), no. 843. Zbl 1093.20025 MR 2182268

[27] P. Tukia, A remark on the paper by Floyd. In D. Drasin, C. J. Earle, F. W. Gehring, I. Kra, and A. Marden (eds.), Holomorphic functions and moduli. (Berkeley, CA, 1986) Springer, New York, 1988, 165-172. Zbl 0646.00005 (collection) MR 0955827 (collection)

[28] P. Tukia, Convergence groups and Gromov's metric hyperbolic spaces. New Zealand J. Math. 23 (1994), no. 2, 157-187. Zbl 0855.30036 MR 1313451

[29] P. Tukia, Conical limit points and uniform convergence groups. J. Reine Angew. Math. 501 (1998), 71-98. Zbl 0909.30034 MR 1637829

[30] A. Weil, Sur les espaces à structure uniforme et sur la topologie générale. Actual. sci. industr. 551. Université de Strasbourg, Strasbourg, 1937. JFM 63.0569.04

[31] A. Yaman, Topological characterisation of relatively hyperbolic groups. J. Reine Angew. Math. 566 (2004), 41-89. Zbl 1043.20020 MR 2039323

[32] W. Yang, Peripheral structures of relatively hyperbolic groups. J. Reine Angew. Math. 689 (2014), 101-135. Zbl 1298.20053 MR 3187929

Received July 29, 2011

Victor Guerassimov, Departamento de Matemática, Universidade Federal de Minas Gerais, Av. Antonio Carlos, 6627, Caixa Postal 702, 31270-901 Belo Horizonte, MG, Brasil

e-mail: victor@mat.ufmg.br

Leonid Potyagailo, UFR de Mathématiques, Université de Lille 1, 59655 Villeneuve d'Ascq cedex, France

e-mail: potyag@math.univ-lille1.fr 